UCRL- $-50027-81$

DE\&2 016138

\title{
Environmental Monitoring at the Lawrence Livermore National Laboratory 1981 Annual Report
}

\author{
M. Auyong \\ K. S. Griggs \\ R. W. Buddemeier \\ Manuscript Date: March 11, 1982

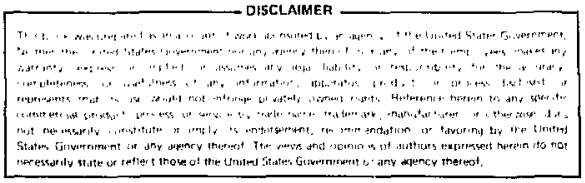

\section{LAWRENCE LIVERMORE LABORATORY University of California - Livermore, California • 94550}




\section{Foreword}

This report is prepared for the U. S. Department of Energy by the Environmental Evaluations Group of the Hazards Control Department, Lawrence Livermore National Laboratory. Data are obtained through the combined efforts of the Nuclear Chemistry Division and the Hazards Control Department. In addition to the authors listed, the following personnel made significant contributions to this report.
V. E. Arganbright
M. W. Nathans
J. M. Bazan
K. R. Peterson
H. C. Capwell
D. Prokosch
A. Conover
D. E. Reed
K. J. Davidson
N. H, Rogers
R. J. Dupzyk
M. R. Ruggieri
L. A. Fry
G. L. Seibel
A. L. Gazlay
C. W. Sundbeck
A. R. Grayson
R. D. Szidon
C. L. Lindeken
C. R. Veith
M. A. Loquist
S. R. Velen
W. A. McConachie
D. K. Wadsworth
F. M. McMillen 


\section{Contents}

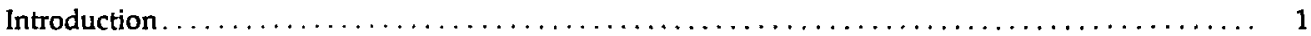

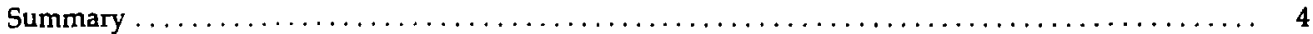

Monitoring Data--Collection, Analysis, and Evaluation $\ldots \ldots \ldots \ldots \ldots \ldots \ldots \ldots \ldots \ldots \ldots \ldots \ldots$

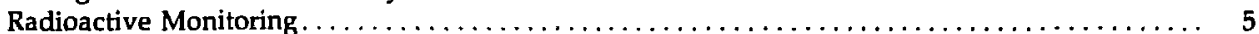

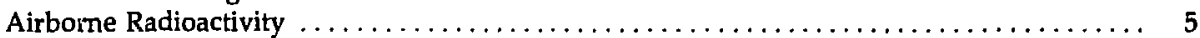

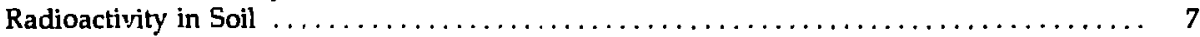

Radioactivity in Sewage $\ldots \ldots \ldots \ldots \ldots \ldots \ldots \ldots \ldots \ldots \ldots \ldots \ldots \ldots \ldots \ldots \ldots \ldots \ldots \ldots$

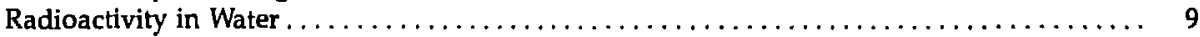

Radioactivity in Vegetation and Foodstuffs $\ldots \ldots \ldots \ldots \ldots \ldots \ldots \ldots \ldots \ldots \ldots \ldots \ldots, 12$

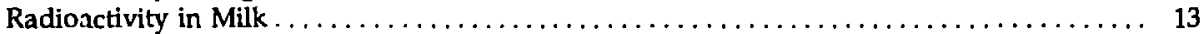

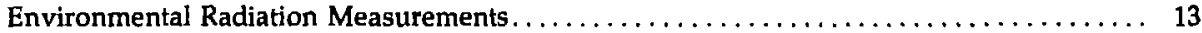

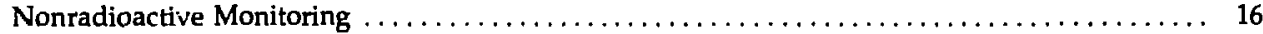

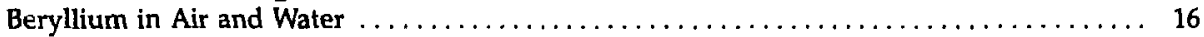

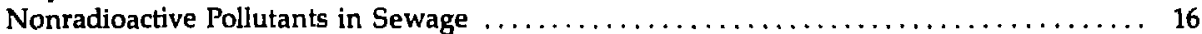

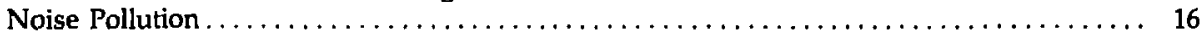

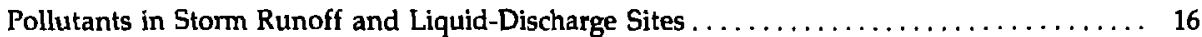

Environmental Impact of LLNL Operations $\ldots \ldots \ldots \ldots \ldots \ldots \ldots \ldots \ldots \ldots \ldots \ldots \ldots \ldots \ldots$

Radioactive Airborne Effluents $\ldots \ldots \ldots \ldots \ldots \ldots \ldots \ldots \ldots \ldots \ldots \ldots \ldots \ldots \ldots \ldots \ldots 17$

Radioactive Liquid Effluents $\ldots \ldots \ldots \ldots \ldots \ldots \ldots \ldots \ldots \ldots \ldots \ldots \ldots \ldots \ldots \ldots \ldots \ldots, 17$

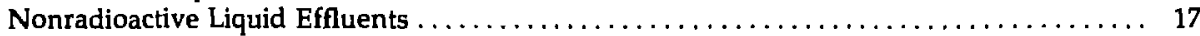

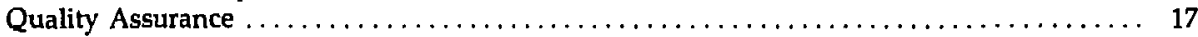

References ............................................. 19

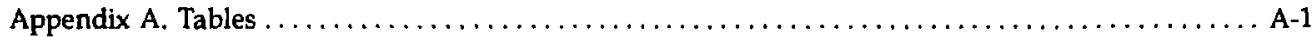

Appendix B. Environmental Activity Concentration-Guide Levels $\ldots \ldots \ldots \ldots \ldots \ldots \ldots \ldots \ldots \ldots$ B-1

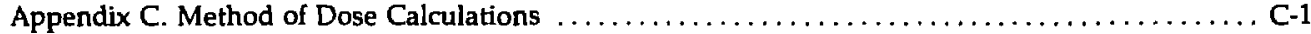

Appendix D. Discharge Limits to the Sanitary Sewer System of Livermore .............. D-1

Appendix E. Sampling and Analytical Procedures for Environmental Monitoring $\ldots \ldots \ldots \ldots \ldots$ E-i 


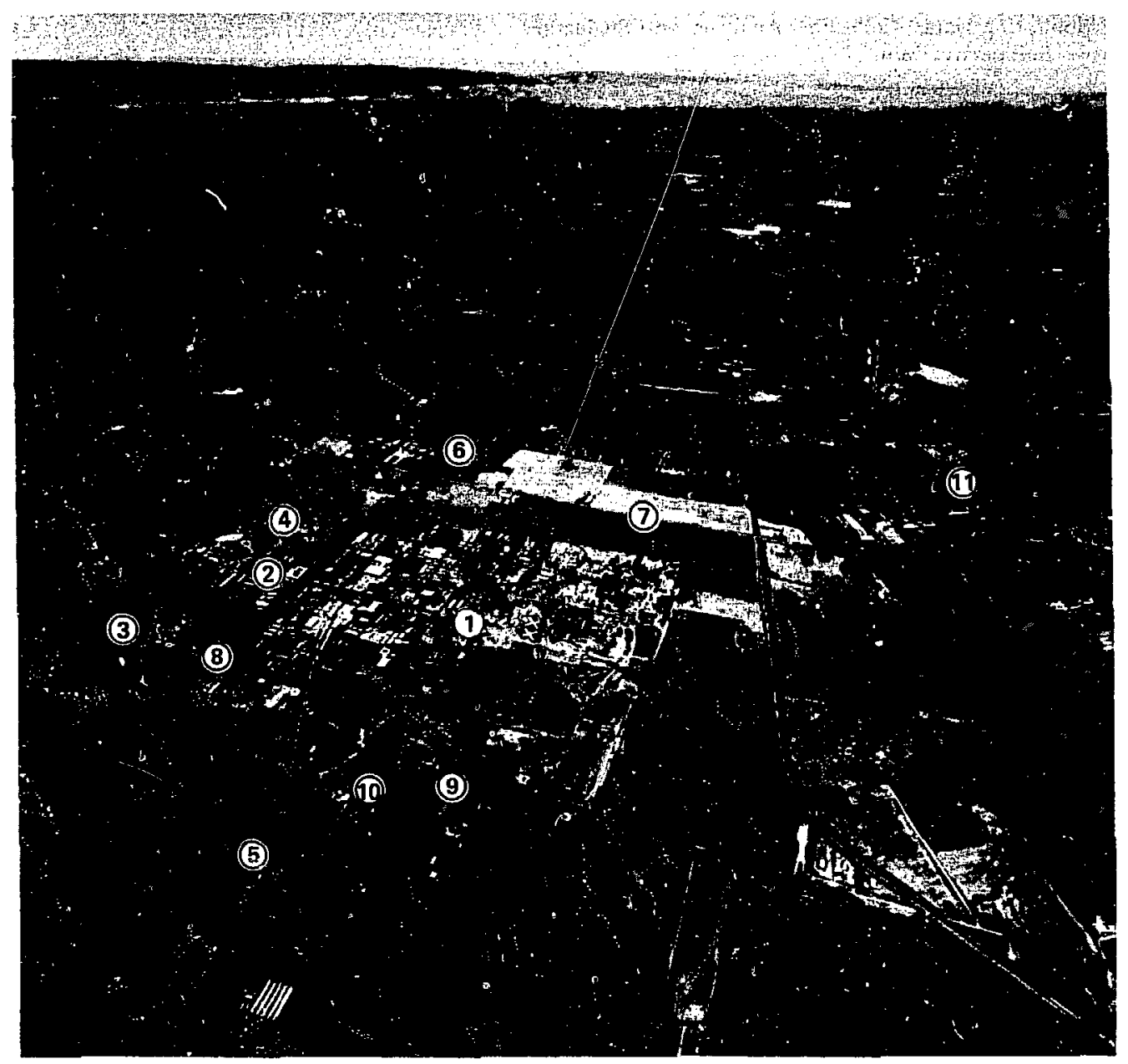
(1) Lawrence Livermore National Laboratory
(7) Vasco Road
(2) Sandia National Laboratories, Livermore
(8) East Avenue
(3) South Bay Aqueduct
(9) Greenville Road
(4) Arroyo Seco
(5) Arroyo Las Positas
(10) Lupin Way
(6) Nearest residential area
(10) Interstate $\mathbf{5 8 0}$

Frontispiece. Lawrence Livermore National Laboratory (looking west). 


\section{Environmental Monitoring at the Lawrence Livermore National Laboratory}

\section{Annual Report}

\section{Introduction}

The Lawrence Livermore National Laboratory (LLNL) is located about $64 \mathrm{~km}$ east of San Francisco, California, in the Livermore Valley of eastern Alameda County, approximately $5 \mathrm{~km}$ east of the city of Livermore. The site, which occupies an area of $2.54 \mathrm{~km}^{2}$, is surrounded by open agricultural areas on the north, east, west, and part of the south side. Sandia Laboratories, Livermore, occupies a portion of the adjoining property on the south, and the nearest residential area is $0.8 \mathrm{~km}$ from the Laboratory's west perimeter. Of the nearly 4.8 million people who live within 80 $\mathrm{km}$ of the Laboratory (Fig. 1), 50,000 live in Livermore.

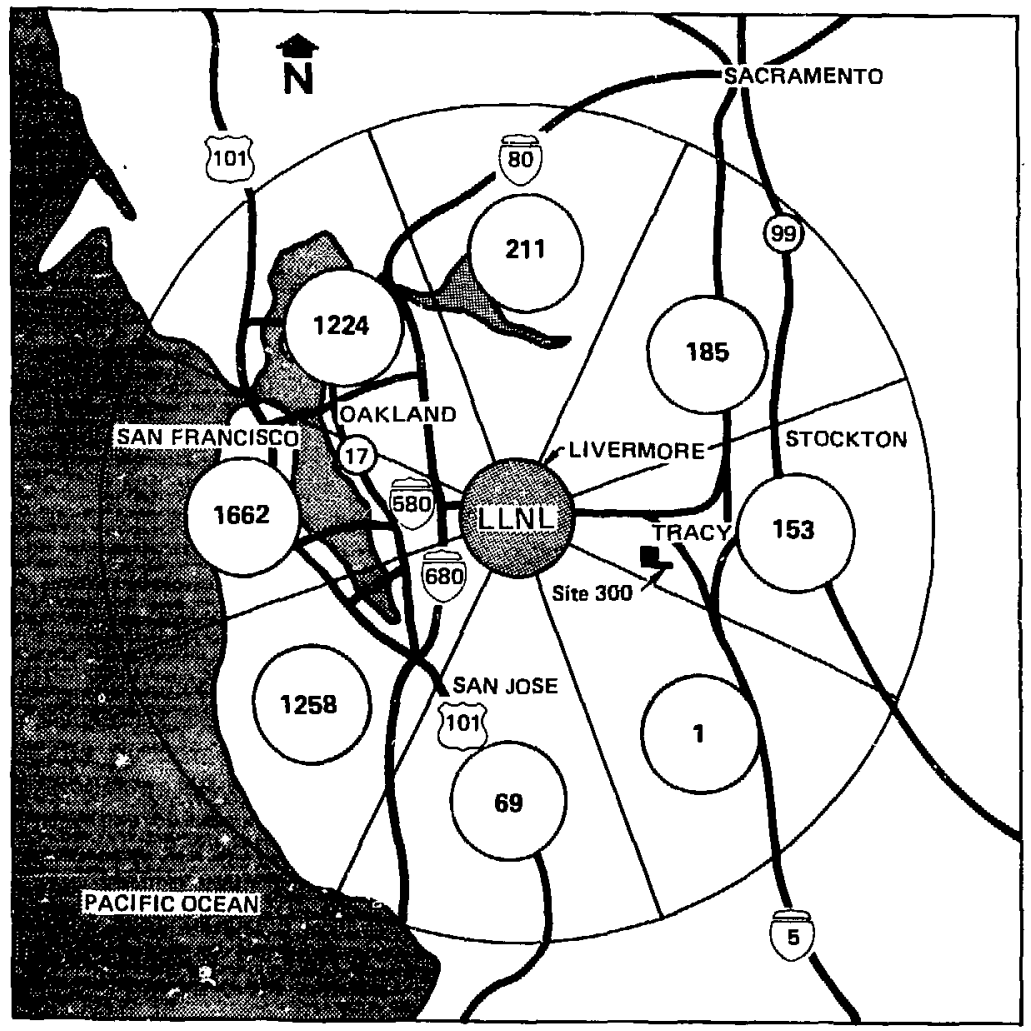

Figure 1. Estimated population distribution (in thousands) within $80 \mathrm{~km}$ of Livermore, by sectors. 
Established in 1952, the Laboratory is operated for the Department of Energy (DOE) by the University of California; in 1981 it employed approximately 7700 people. Although nuclear weapons research and development has always been the primary mission of LLNL, additional programs include biomedical studies, magneticfusion and laser-fusion energy research, and nonnuclear energy technologies.

Much of the Laboratory's materials testing and high-explosives diagnostic work is conducted at Site $300,16 \mathrm{~km}$ southeast of Livermore. Located in the sparsely populated hilis of the Diablo Range, Site 300 covers an area of $27 \mathrm{~km}^{2}$. Figure 1 shows the location of LLNL and Site 300 with respect to the city of Livermore and surrounding areas.

The Livermore Valley has a climate characterized by mild, rainy winters and warm, dry summers. Annual rainfall averages about $360 \mathrm{~mm}$, and rains occur predominantly between November and April, usually in connection with Pacific storms. Rainfall for the 1980-1981 season (July 1, 1980, through June 30,1981 ) was $263 \mathrm{~mm}$. By comparison, during the two drought periods, 1975-76 and 1976-77, rainfall totals were 138 and $196 \mathrm{~mm}$, respectively. Surface water drainage from the Vallay is from east to west through various arroyos; the outfall is near Sunol in the southwest corner of the Valley. Prevailing winds are from the west and southwest during April through September. During the remainder of the year, wind directions are variable, as shown by the wind rose in Fig. 2 .

The Livermore site is situated on a northwesterly sloping alluvial flood plain bordering the low hills of the Livermore Uplands to the south. Groundwater is found at depths of 15 to $30 \mathrm{~m}$ below the LLNL site with a gradient indicating a generally westward flow. The lithology of the area consists of a series of unconsolidated marine and continental sedimentary units such as sandstones, gravels, silts, and clays overlying the interbedded sandstones of the Franciscan Formation. The hilly terrain surrounding the Valley is used for cattle and sheep pasture, and the principal agricultural products in the vicinity of LLNL are grapes and wine, cattle, and poultry.

Water bodies adjacent to the Laboratory include the South Bay Aqueduct, which runs from the northeast to southwest, $1.8 \mathrm{~km}$ to the southeast; the Patterson Pass water treatment facility, about $2 \mathrm{~km}$ east of LLNL; and Frick Lake, $4 \mathrm{~km}$ north of LLNL, a sag pond that is dry most of the year. Aquatic recreation (boating, fishing, and swimming) is available at Lake Del Valle, about $8 \mathrm{~km}$ south of LLNL, and at the Shadow Cliffs Recreation Area, $11 \mathrm{~km}$ to the west.

The Laboratory normally receives its treated water from the Hetch Hetchy Aqueduct (which supplies San Francisco), located $11 \mathrm{~km}$ southwest of Livermore.

Laboratory storm water is channeled through storm sewers designed to accommodate a 10-year flow. Open ditches are used in undeveloped areas of the site. Arroyo Seco crosses LLNL at the southwest corner. Arroyo Ias Positas originally crossed the northeast section of the site. However, in 1965, as part of an erosion-control program, Arroyo Las Positas was channeled north to the northeast corner of the site, and then west along the north perimeter to an outlet at the northwest corner. This outlet, which also constitutes the main pathway for the Laboratory's surface drainage (storm and irrigation), runs north to the Western Pacific tracks, then west where it joins Arroyo Seco. The LLNL Master Site Plan calls for a small lake to be established in the center of the project. Provisions have been made for rerouting on-site water drainage and the Arroyo Las Positas to fill this lake during the rainy season.

Laboratory sewage is discharged into the City of Livermore's sanitary sewer system and processed at the Livermore Water Reclamation Plarit (LWRP). As part of the Livermore-Amador Valley Wastewater Management program, the treated sanitary wastewater is transported out of the valley via a pipeline and discharged into the San Francisco Bay. The LWRP was connected to this pipeline on February 8, 1980. While the LWRP effluent is still used for summer irrigation of nearby Livermore city property, it is no longer discharged to Arroyo Las Positas, as was done during the wet season before construction of the pipeline.

A strict effluent-control program that emphasizes controlling effluents at the source has been in effect since the Laboratory began operation. The environmental monitoring program is maintained to evaluate the effectiveness of these measures, to document whether effluents from the Laboratory and Site 300 operations are within applicable standards, and to estimate the impact of these operations on the environment. Sensitive monitoring equipment is used that can detect radioactive and nonradioactive pollutants well below environmental background levels. The program includes the collection and analysis of air, 


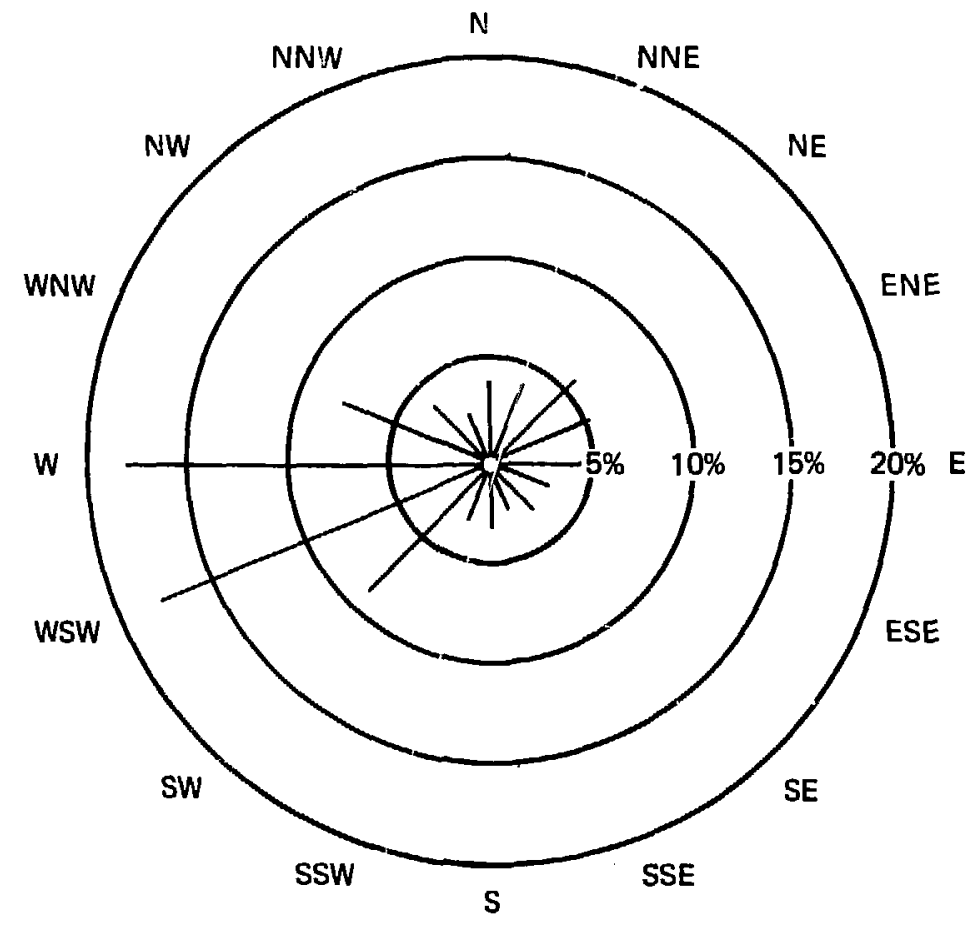

Average annual percent frequency of wind direction vs wind speed

\begin{tabular}{|c|c|c|c|c|c|c|c|c|c|c|c|}
\hline \multirow[b]{2}{*}{ Direction } & \multicolumn{9}{|c|}{ Speed $(\mathrm{m} / \mathrm{s})$} & \multirow[b]{2}{*}{ Total } & \multirow[b]{2}{*}{ Average } \\
\hline & $0-2$ & $2-3$ & $3-5$ & $5-7$ & $7-9$ & 9.11 & $11-16$ & $16-20$ & $>20$ & & \\
\hline $\mathbf{N}$ & 2.2 & 0.5 & 0.5 & 0.2 & 0.2 & 0 & 0.1 & 0 & 0 & 3.7 & 2.9 \\
\hline NNE & 2.1 & 0.6 & 1.1 & 0.6 & 0.3 & 0.1 & 0 & 0 & 0 & 4.9 & 3.1 \\
\hline NE & 2.1 & 0.9 & 2.0 & 0.7 & 0.1 & 0 & 0 & 0 & 0 & 5.7 & 3.0 \\
\hline ENE & 1.9 & 0.7 & 1.3 & 0.9 & 0.3 & 0 & 0 & 0 & 0 & 5.2 & $\mathbf{3 . 3}$ \\
\hline$E$ & 1.8 & 0.9 & 1.1 & 0.5 & 0.2 & 0 & 0 & 0 & 0 & 4.5 & 3.0 \\
\hline ESE & 1.7 & 0.4 & 0.3 & 0 & $\mathbf{0}$ & 0 & 0 & 0 & 0 & 2.4 & 1.7 \\
\hline SE & 1.6 & 0.3 & 0.2 & 0 & 0 & 0 & 0 & 0 & 0 & 2.1 & 1.7 \\
\hline SSE & 1.4 & 0.2 & 0.1 & 0 & 0 & 0 & 0 & 0 & 0 & 1.8 & 1.6 \\
\hline $\mathbf{s}$ & 1.9 & 0.4 & 0.3 & 0.1 & 0.1 & 0 & 0 & $\mathbf{0}$ & 0 & 2.9 & 2.1 \\
\hline SSW & 2.3 & 0.8 & 0.5 & 0.2 & 0.1 & 0 & 0 & 0 & 0 & 4.0 & 2.4 \\
\hline SW & 3.1 & 1.4 & 2.0 & 1.5 & 0.4 & 0.1 & 0 & 0 & 0 & 8.4 & 3.3 \\
\hline WSW & 3.3 & 3.0 & 6.1 & 3.9 & 1.2 & 0.1 & 0 & 0 & 0 & 17.7 & 4.0 \\
\hline$w$ & 2.7 & 2.8 & 5.7 & 4.3 & 1.6 & 0.3 & 0.1 & 0 & 0 & 17.3 & 4.3 \\
\hline WNW & 2.0 & 1.3 & 2.1 & 1.7 & 0.5 & 0.1 & 0 & 0 & 0 & 7.7 & 3.8 \\
\hline NW & 1.6 & 0.6 & 0.6 & 0.7 & 0.2 & 0 & 0 & 0 & 0 & 3.8 & 3.2 \\
\hline NNW & 1.8 & 0.3 & 0.2 & 0 & 0 & 0 & 0 & 0 & 0 & 2.4 & 1.6 \\
\hline Calm & & & & & & & & & & 5.6 & \\
\hline Total & 33.6 & 15.0 & 24.2 & 15.5 & 5.1 & 0.7 & 0.3 & 0 & 0 & 100.0 & 3.2 \\
\hline
\end{tabular}

Figure 2. Wind rose showing average annual wind direction and speed during 1981 (measurements are made at $\mathbf{4 0} \mathrm{m}$ above ground). 
soil, water, sewer effluent, vegetation, foodstuffs, and milk samples. Environmental background radiation is measured at numerous locations in the vicinity of the Laboratory using gamma and neutron dosimeters.

Each spring, the Laboratory reports the results of environmental monitoring for the previous year. This report is prepared in compliance with the DOE Manual 5484.1, Environmental Protection, Safety, and Health Protection Information Reporting Requirements. Significant changes in either the scope of the program or the levels of effluents are noted.

Appendix A is a tabulation of 1981 environmental monitoring data. Graphics have been used in the body of the report to aid in interpretation.
When appropriate, the tabulated data contain maximum, minimum, and average values. Radioactivity values are tabulated with the associated counting uncertainties at the $2 \sigma$ ( $95 \%$ confidence) level. Unless otherwise stated, the minimum detection limit of these measurements is assumed to have been reached when the $2 \sigma$ error is $\pm 100 \%$. In the case of radioactivity measurements, an attempt has been made to assess the potential impact on man of the observed environmental levels of artificially produced radionuclides. This assessment is made by calculating the whole-body or critical-organ doses delivered to man and comparing these with the much larger radiation dose received locally from natural sources.

\section{Summary}

In 1981, the annual average airborne gross beta activity in Livermore Valley air samples was $9.2 \times 10^{-14} \mu \mathrm{Ci} / \mathrm{ml}$, which was greater than the level cbserved in 1980. This is attributed to fallout from a nuclear iest by the People's Republic of China on October 16,1980, which increased beta airborne concentrations through the first three quarters of 1981 . Airborne ${ }^{238} U$ concentrations at Site 300 were higher than those at Livermore because of the "depleted" uranium (a byproduct of ${ }^{235} \mathrm{U}$ enrichment) used in high-explosive tests at the Site. However, these concentrations were well below the standards set by DOE. The average gross alpha activity shows no significant change from 1980 data. The average annual beryllium concentrations were less than $4 \%$ of the appropriate standard at both the Laboratory perimeter and Site 300 . The beryllium concentrations can be accounted for by the natural beryllium in typical airborne dust.

Water samples collected in the Livermore Valley and at Site 300 exhibit gross beta and tritium activities within the ranges previously observed in these areas. Two water samples from Site 300 showed an above-average level of gross alpha activity. This activity has been found to be naturally occurring uranium at concentrations well within those specified in DOE Manual 5480.1 .

The release of HTO into the sanitary sewer system was $6 \mathrm{Ci}$ in 1981 compared with $5 \mathrm{Ci}$ in 1980. Tritium analyses were also made on well-water samples collected near the Livermore Water Reclamation Plant (LWRP). These analyses, begun in 1977, were made to determine the extent to which low levels of tritium in the LWRP effluent may be migrating into groundwater. As was found during earlier surveys, the highest tritium values were detected in test wells west of the site near Arroyo Las Positas. Since the LWRP effluent is no longer discharged to the arroyo, the primary means of tritium movement to nearby groundwater has been eliminated. Consequently, tritium concentrations observed in local wells in 1981 were generally lower than previous annual measurements.

The total tritium released to the atmosphere in 1981 was $2620 \mathrm{Ci}$. The concentration of tritium in air was noticeably higher in December due to a release from the tritium facility; however, the annual average was similar to that of the previous year.

Tritium levels found in the Livermore Valley and Site $\mathbf{3 0 0}$ vegetation were comparable to those observed in 1980. As a means of evaluating the possible impact of Laboratory effluents on locally grown foodstuff, the tritium content of Livermore Valley wines was compared with values from other California and European wines. The tritium levels in Valley wines are within the range found to be present in both European uines and surface waters throughout the world, but somewhat higher than those in other California wines. Honey produced in the Livermore Valley contained tritium levels comparable to those found in honey from neighboring areas.

The 1981 median annual gamma-radiation dose at the Laboratory perimeter was 54 mrem, 
and the median off-site background dose was 57 mrem. A 14-MeV neutron generator (Bldg. 212) near the south perimeter continued to be a source of elevated radiation. However, the annual radiation dose at the Laboratory's south perimeter is well below standards in DOE Manual 5480.1.

\section{Monitoring Data-Collection, Analysis, and Evaluation}

\section{Radioactive Monitoring}

\section{Airborne Radioactivity}

Concentrations of various airborne radionuclides are measured at the Laboratory perimeter, at off-site locations near the Laboratory, and at Site 300. Sampling locations are shown in Figs. 3 through 14. The six samplers on the Laboratory perimeter (Fig. 3) and the nine samplers at Site 300 (Fig. 4) use $5.2 \times 10^{-2} \mathrm{~m}^{2}$ Whatman-4. - $21 \mathrm{lu}-$ lose filters. These samplers are operated at average flow rates of 400 to 700 liters/min. The filters are cut in half; one half of each filter is retained for beryllium analysis. An easily dissolved filter and a low trace-metal background are requirements for these analyses. Whatman 41 represents a balance between such requirements and particulate-collection efficiency. ${ }^{1}$ Off-site samplers throughout the Livermore Valley (Fig. 5) use 4.5b $\times 10^{-3} \mathrm{~m}^{2}$ glass-fiber filters (Flanders F-700) and

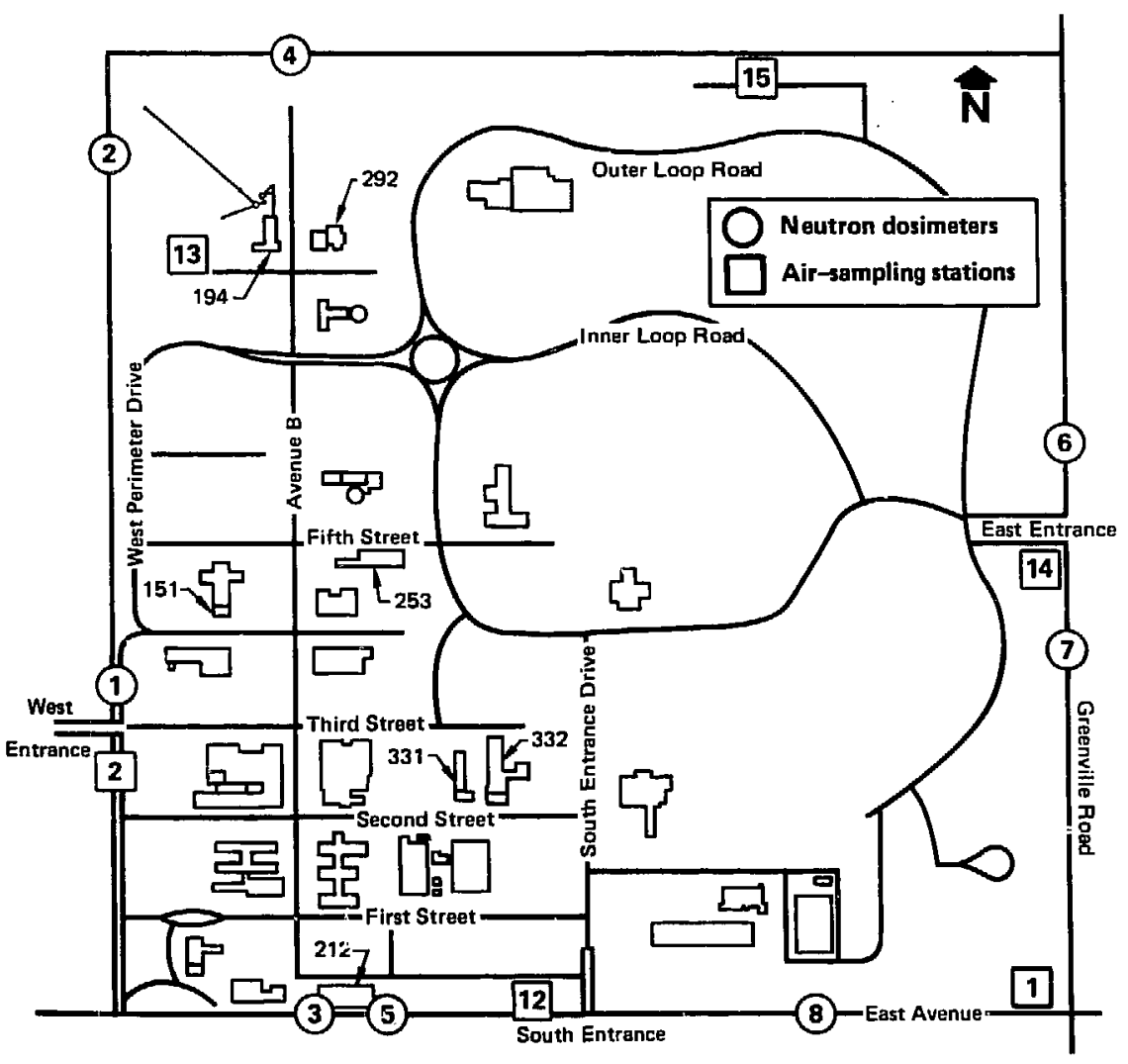

Figure 3. LLNL perimeter locations for air-sampling stations and neutron dosimeters. 


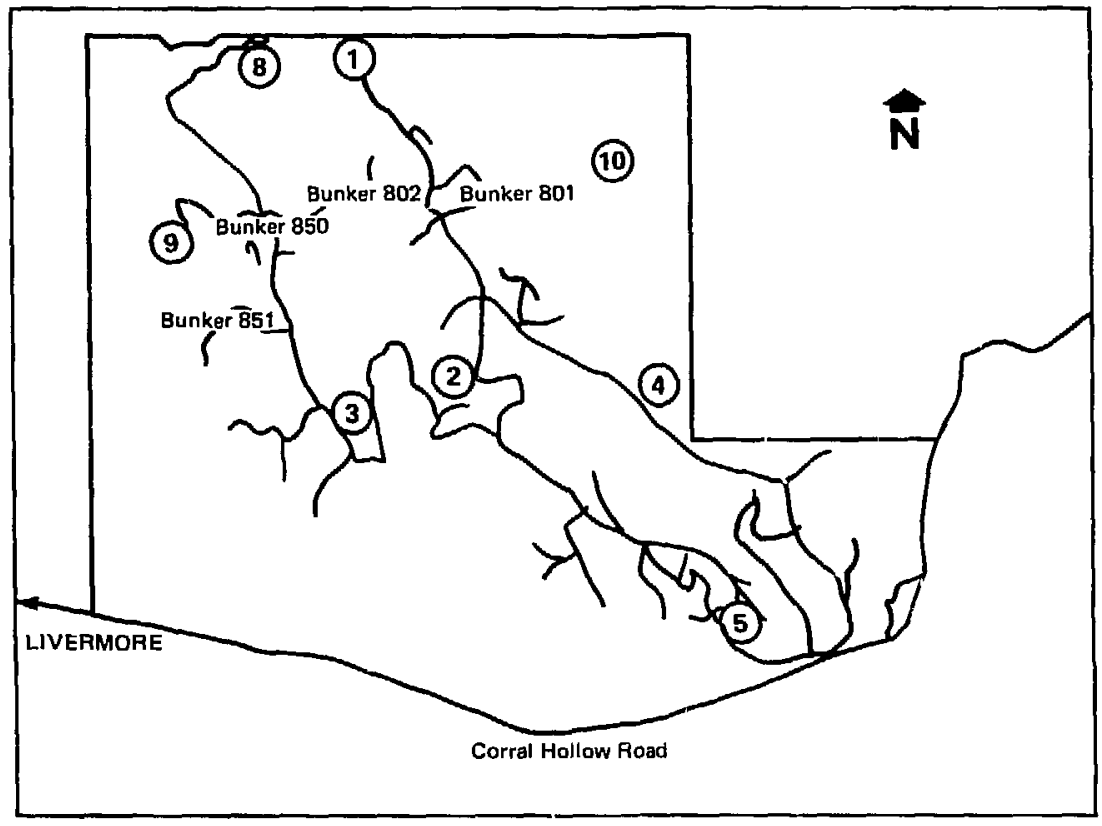

Figure 4. Site 300 on-site air-sampling locations.

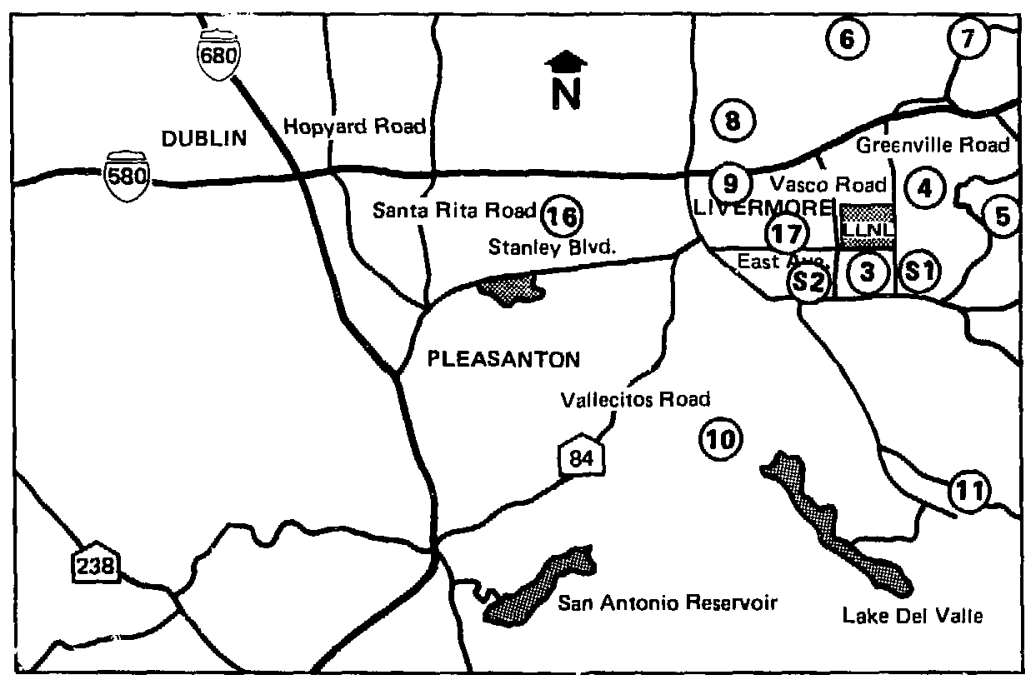

Figure 5. Livermore Valley air-sampling locations. 
are operated at a flow rate of 80 liters $/ \mathrm{min}$. All air filters are changed weekly.

After a four-day delay for decay of the radon-thoron daughters, gross alpha and beta activities on the filters are determined with an automatic gas-flow proportional counter. Monthly composites of Laboratory perimeter and Site 300 filters are also counted ior specific gammaemitting radionuclides using low-background $\mathrm{Ge}(\mathrm{Li})$ detectors. Following gamma counting, the Laboratory perimeter filters from individual locations and the Site 300 composites are analyzed for the presence of ${ }^{239} \mathrm{Pu},{ }^{238} \mathrm{Pu},{ }^{235} \mathrm{U}$, and ${ }^{238} \mathrm{U}$.

Tables 1 and 2 in Appendix A show airborne gross alpha and gross beta activities for the Livermore Valley samples, and Tables 3 and 4 show corresponding activities for Site 300 . Average annual gross beta activity in both Livermore and Site 300 air samples was about three times higher than that observed during 1980 . This increase occurred througnout the first three quarters and is attributed to fallout from a nuclear test by the People's Republic of China on Dctober 16, 1980. The average gross alpha activity shows no significant change from the 1980 data.

Tables 5 and 6 list the activities of the principal radionuclides that contribute to the gross beta activity in Livermore and Site 300 samples. These activities are detennined by gamma spectra analysis. The increase in fission products seen on the January to September air filters is a result of successive passes of debris from the Chinese test. Table 7 shows the concentration of plutonium on air filter samples collected in Tracy and the Livermore Valley. Tables 8 and 9 show the concentrations of irborne ${ }^{239} \mathrm{Pu},{ }^{235} \mathrm{U}$, and ${ }^{258} \mathrm{U}$ in the Livermore $P E$-imeter and Site 300 air samples. The concentrations of ${ }^{235} U$ and ${ }^{238} U$ showed an increase at Livermore and Site 300 in December. We have observed this occurrence in past years. The higher concentration of ${ }^{238} U$ at Site 300 is a result of the "depleted" uranium (a byproduct of ${ }^{235} \mathrm{U}$ enrichment) used at the Site. The uranium concentrations are well below the concentration guides (CG) in DOE Manual 5480.1 (see Appendix B).

Concentrations of airborne tritiated water (HTO) were determined for each of the LLNL perimeter air-sampling locations (Fig. 3) and at off-site locations $\mathrm{S} 1$ and S2 (- $\mathrm{F}_{\mathrm{ig}}$. 5). Water vapor was collected on silica-gei samplers that operated at a flow rate of about 0.5 liters/min for a 2 -week period. The collected water was recovered by vacuum drying the silica gel at $150^{\circ} \mathrm{C}$, and the HTO was measured by liquid-scintillation counting.
Table 10 shows average monthly HTO concentrations observed at each sampling location; the overall annual average concentration is $4.4 \times$ $10^{-11} \mu \mathrm{Ci} / \mathrm{ml}$. All locations showed higher concentrations in December reflecting an increase in HTO released from the tritium facility, Building 331 , during the month. The highest December concentration, which was also the highest monthly HTO concentration, was observed at location 15 at the north site boundary. This concentration, $39 \times 10^{-11} \mu \mathrm{Ci} / \mathrm{ml}$, is $0.2 \%$ of the $\mathrm{DOE}$ standard. Location 12 showed the highest annual average concentration, probably because this location is so close to one of the tritium effluent sources (the 14-MeV zeutron generator).

\section{Radioactivity in Soil}

An intensive soil-sampling program conducted in 1971 and 1972 provided a data base on the concentration ranges of various radionuclides in soil near the Laboratory and at Site 300 . Since 1972, soil sampling in the vicinity of LLNL and at Site 300 has been part of a continuing LLNL surveillance program to document any changes in environmental levels of radioactivity that may have occurred and to evaluate any increase in radioactivity that might have resulted from Laboratory operations. As in previous years, a group of soil samples was collected at random from the listing of locations sampled in the 1971-1972 study. Figure 6 shows the 1981 soil-sampling locations in the Livermore Valley and Fig. 7 indicares the locations at Site 300 where annual samples have been collected since 1973 .

All samples were collected to a depth of $5 \mathrm{~cm}$. After collection, the samples were dried, ground, and blended. Plutonium-239 content was determined in each sample following an acid-leaching procedure. For gamma-spectra analysis, approximately $300-g$ aliquots of soil were sealed in 200 $\mathrm{cm}^{3}$ thin-walled cans of aluminum and counted with a $\mathrm{Ge}(\mathrm{Li})$ detector.

The data in Table 11 are in the range of those reported in the previous year. ${ }^{2}$ Figure 8 is a distribution plot of these activities.

Table 12 shows the ${ }^{239} \mathrm{Pu},{ }^{137} \mathrm{Cs}$, and ${ }^{238} \mathrm{U}$ activities in the Site 300 samples. There were negligible changes from activities observed in previous samples collected in the same locations. ${ }^{2}$

High-explosive tests at Site 300 often involve the use of depleted uranium. Accordingly, soil samples are taken annually to determine how these tests perturb the ${ }^{235} \mathrm{U} /{ }^{238} \mathrm{U}$ ratio of the soil. Isotopic uranium measurements were made with isotopic-dilution mass spectrometry. As in the 


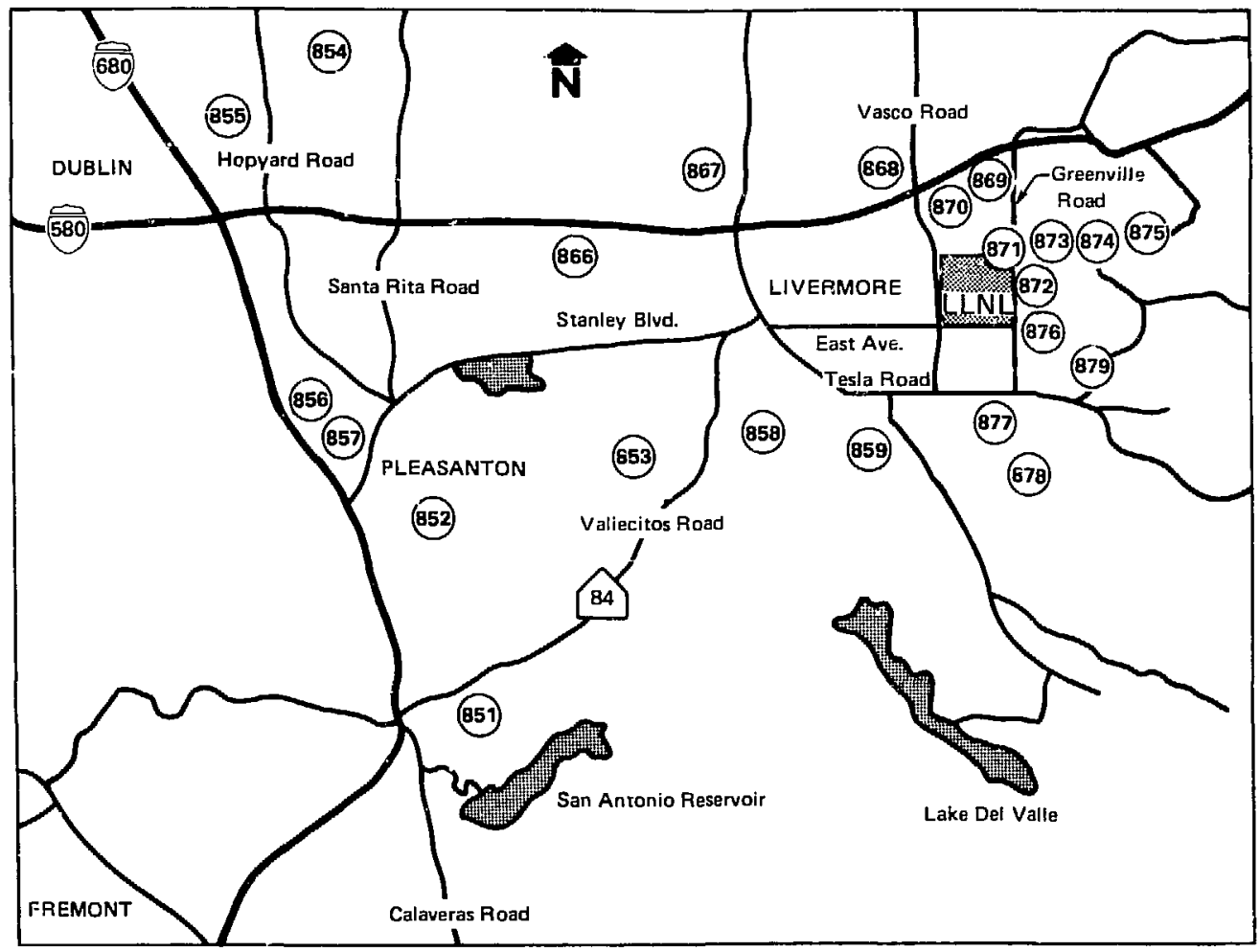

Figure 6. Livermore Valley soil-sampling locations.

past, the analyses indicated that isotopic perturbation is essentially limited to areas adjacent to the firing bunkers. ${ }^{3,4}$ The isotopic uranium data shown in Table 12 are comparable to those observed during 1980.

\section{Radioactivity in Sewage}

Radioactive wastewater is treated to reduce activity levels to the lowest levels practicable and well below standards in DOE Manual 5480.1. After treatment and analysis, the liquid effluents are released into the city of Livermore's sanitary sewer system at the outfall located at the northwest corner of the LLNL. The effluent is continuously monitored at the outfall for $\mathrm{pH}$ and radioactivity.

Liquid wastes from Livermore's sanitary sewer system are treated at the Livermore Water Reclamation Plant (LWRP), a tertiary sewagetreatment plant that serves residential, commercial, and industrial water users in Livermore. The salt content of the LWRP effluent presented a s roblem for groundwater quality locally and in the Niles Cone aquifers. A ccordingly, as part of the Livermore-Amador Valley Wastewater Management program, a pipeline was constructed to transport wastewater out of the valley for discharge into the San Francisco Bay. The Livermore sewage plant was connected to this pipeline on February 8, 1980. Although the effluent will still be used for irrigation of municipal property during summer months, it will no longer be discharged into the anroyo, thus eliminating the primary means of tritium migration to local and downstream groundwater.

Representative San Francisco Bay water samples were collected before and after the LWRP's connection to the pipeline. Results of tritium analysis of these samples indicate no significant difference between the two sample sets. ${ }^{5}$

Samples of the LLNL and LWRP liquid effluents are collected daily. Table 13 compares 


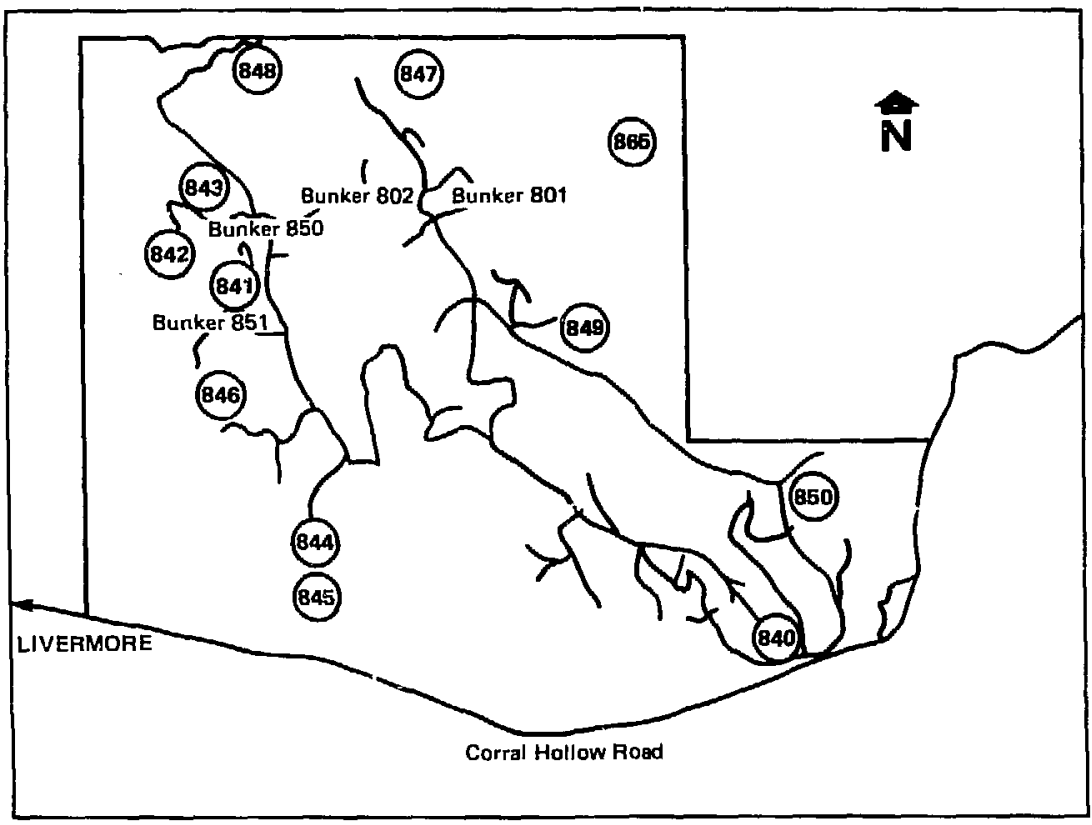

Figure 7. Site $\mathbf{3 0 0}$ soil-sampling locations.

the monthly composite activity levels of certain radionuclides in the LLNL effiuent with those in the effluent from the LWRP. All concentrations are well below standards in DOE Manual 5480.1 for discharge into the sanitary sewer system.

\section{Radioactivity in Water}

Water samples are collected from the various Livermore Valley and Site $\mathbf{3 0 0}$ locations shown in Figs. 9 and 10. These samples are analyzed for gross alpha and gross beta activitiy. Tables 14 and 15 show the gross alpha activities in Livermore Valley and Site 300 samples, respectively. Gross beta activities for Livermore and Site $\mathbf{3 0 0}$ samples are shown in Tables 16 and 17.

Livermore sampling locations 11, 16, 24, 26, and 29 (Fig. 9) are surface water sources (ponds, creeks, and reservoirs), and location 20 (Fig. 10) is the collection site of Site 300 rainfall. The other locations are domestic water sources. Gross alpha and beta activities in Livermore water samples collected in 1981 were below EPA and State of California standards for drinking water. ${ }^{6}$

Storm-drain influent and effluent from the first major rain of the 1981 season were collected. The gross alpha and gross beta from both samples were less than the limit of sensitivity. The tritium concentration in the effluent was $1.5 \times 10^{-6}$ $\mu \mathrm{Ci} / \mathrm{ml}(<0.05 \%$ of the concentration guide).

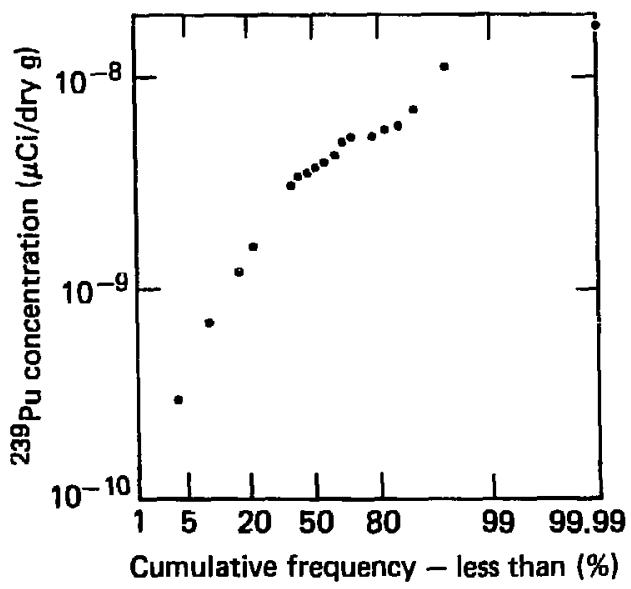

Figure 8. Distribution plot of ${ }^{239} \mathrm{Pu}$ in soil sampies collected in the Livermore Valley during 1981. 


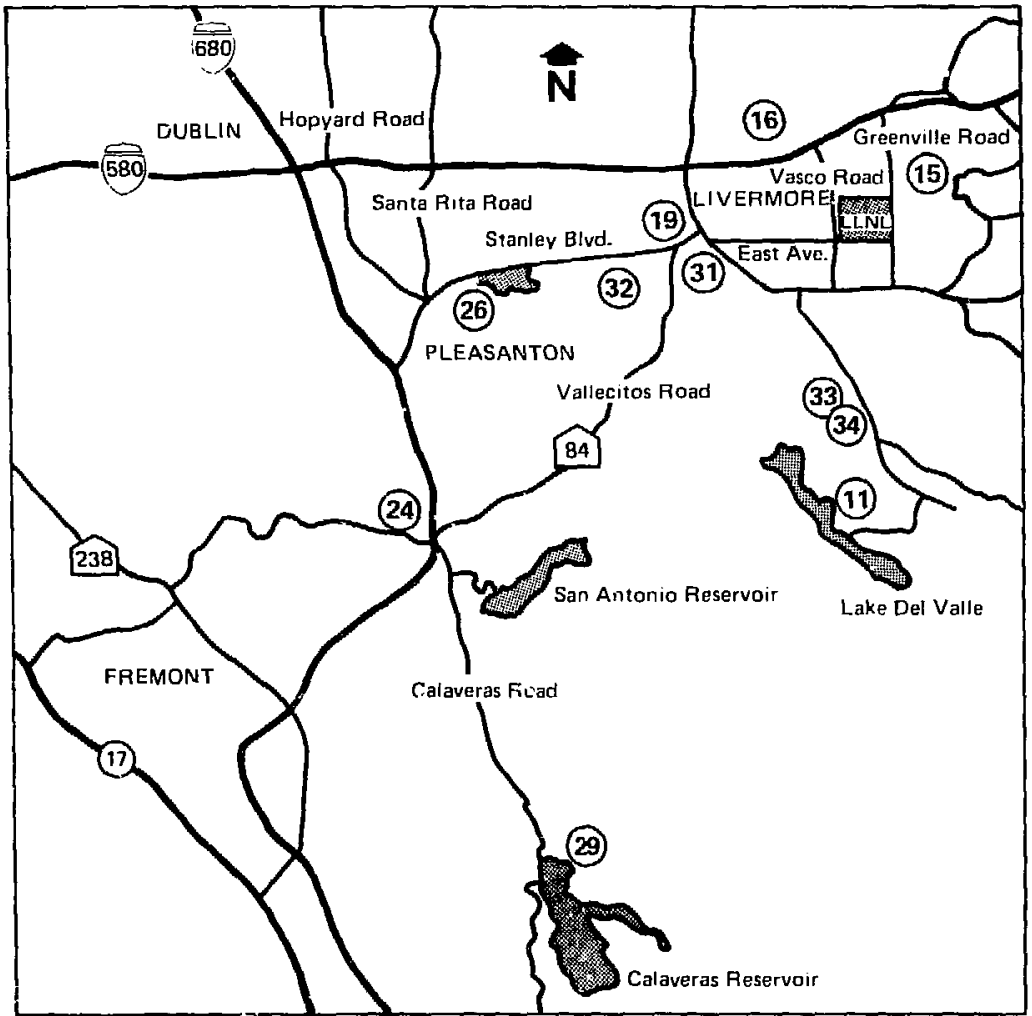

Figure 9. Livermore Valley water-sampling locations.

Site 300 water samples (Fig. 10) are collected from on-site wells (locations 1-6), off-site wells (locations 7 and 11), and an off-site creek (location 14). Location $\mathbf{2 0}$ is Site $\mathbf{3 0 0}$ rainwater, and location 21 is a spring-fed pond near Bunker 812. Two locations showed high gross alpha concentration which specific analyses have identified to be natural uranium: location 4 , a well near the Site 300 entrance, has displayed these results in previous years; location 21, the spring-fed pond, was investigated during 1981. Niveither the $\mathrm{EPA}^{6}$ nor the State of California ${ }^{7}$ specify a limit for natural uranium in drinking wates. In their limit for gross alpha activity, urani:um is specifically excluded from the total. The uranium concentrations were well within that specified in DOE Manual 5480.1 $\left(2 \times 10^{-5} \mu \mathrm{Ci} / \mathrm{ml}\right)$.

The water samples from the Livermore Valley and Site $\mathbf{3 0 0}$ are also analyzed for tritium activity. Because of the low tritium levels typically found it is necessary to distill and electrolytically enrich the samples before liquid-scintillation counting. Tables 18 and 19 show the data for the Livernore and Site $\mathbf{3 0 0}$ samples, respectively. The samples have concentrations that are well below recommended concentration guide values. The maximum tritium-in-rainwater sample $\left(151 \times 10^{-7}\right.$ $\mu \mathrm{Ci} / \mathrm{ml}$ ) was collected in December following an increase in HTO released to the atmosphere from Bldg. 331, the tritium facility. Tables 18 and 19 also include an estimate of the annual dose that may be delivered to an adult who consumes water containing the listed tritium concentrations. These doses, which are all less than $\mathbf{0 . 2}$ mrem, are based on a water consumption of 2 liters/day and the dose-conversion factors contained in NRC Reg. Guide 1.109 (see Appendix C).

As noted previously, treated effluent from the LWRP is used to irrigate nearby municipal property. This effluent contains low levels of tritium 


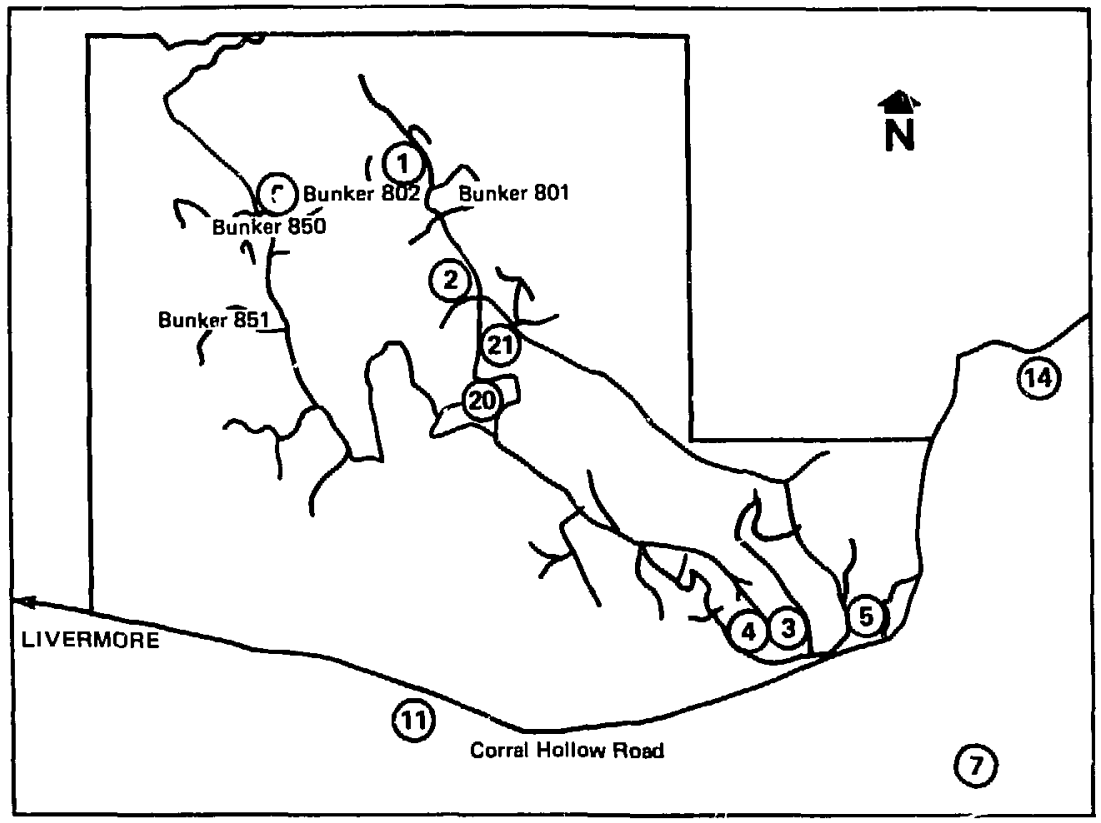

Figure 10. Site 300 water-sampling locations.

which come from normal LLNL operational releases to the sanitary sewer systemi. As part of a study begun in 1977, tritium measurements are now made annually on well-water samples collected from neighboring wells to determine the extent of tritium migration into groundwater.
Many of the wells were in the immediate vicinity of the LWRP; however, additional samples were also collected from areas at some distance. Locations sampled during 1981 are indicared in Fig. 11, and the tritium data are shown in Table 20 . The highest tritium values appear in wells west of the

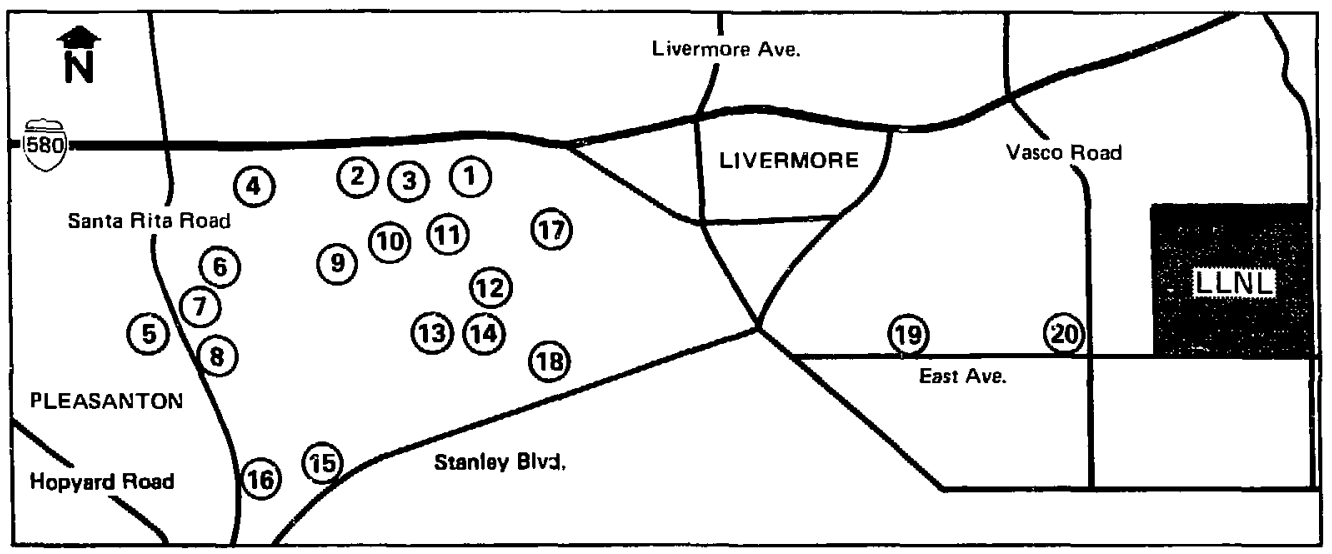

Figure 11. Livermore Valley groundwater-sampling locations. 
LWRP. Tritium activities in all samples were well below the guidelines for water in uncontrolled areas as stated in DOE Manual 5480.1. The tritium levels in these wells are also lower than those observed in the 1980 samples, which is to be expected with diversion of effluent to the pipeline.

\section{Radioactivity in Vegetation and Foodstuffs}

Vegetation samples (usually native grasses) are collected quarterly at locations in the Livermore Valley (Fig. 12) and at Site 300 (Fig. 13). These samples are freeze-dried and the tritium activity in the recovered water is determined by liquid-scintillation counting. Table 21 shows the tritium data for vegetation collected in the Livermore Valley. The whole-body radiation doses shown in Table 21 were derived using the dose-conversion factors in NRC Reg. Guide $1.109,{ }^{9}$ conservatively assuming that all the vegetables an adult consumes have the tritium concentrations found in these unirrigated grasses. These potential doses are all less than $1 \mathrm{mrem} / \mathrm{y}$.

Table 22 shows the 1981 tritium data for Site 300 vegetation. Location 6 is adjacent to an area that contains tritium-contaminated debris from a firing table. As a result of the seasonal rains, the tritium apparently entered an aquifer whose outflow is in the area where locatinn 13 samples are routinely collected. This was also observed in 1980.

Beginning in 1977, as a means of evaluating the possible impact of Laboratory effluents on locally grown foodstuff, the tritium content of Livermore Valley wines was measured and compared with that found in other California wines and European wines. ${ }^{8}$ Wine samples collected in 1981 were catalytically oxidized to carbon dioxide and water, and the tritium content of the recovered water was measured by gas proportional counting. The data in Table 23 represent the analysis of samples purchased in 1981. As found in 1977, the tritium levels of the Valley wines were somewhat higher than those of California wines produced from grapes grown outside the Valley, but lower than that of the European wine sampled. The same European wine source has been sampled in previous years.

Samples of honey produced from a variety of flower sources both in and outside the Livermore Valley were analyzed for tritium content. Following the oxidation of the samples in a Parr oxygen bomb, the tritium content of the wate: produced

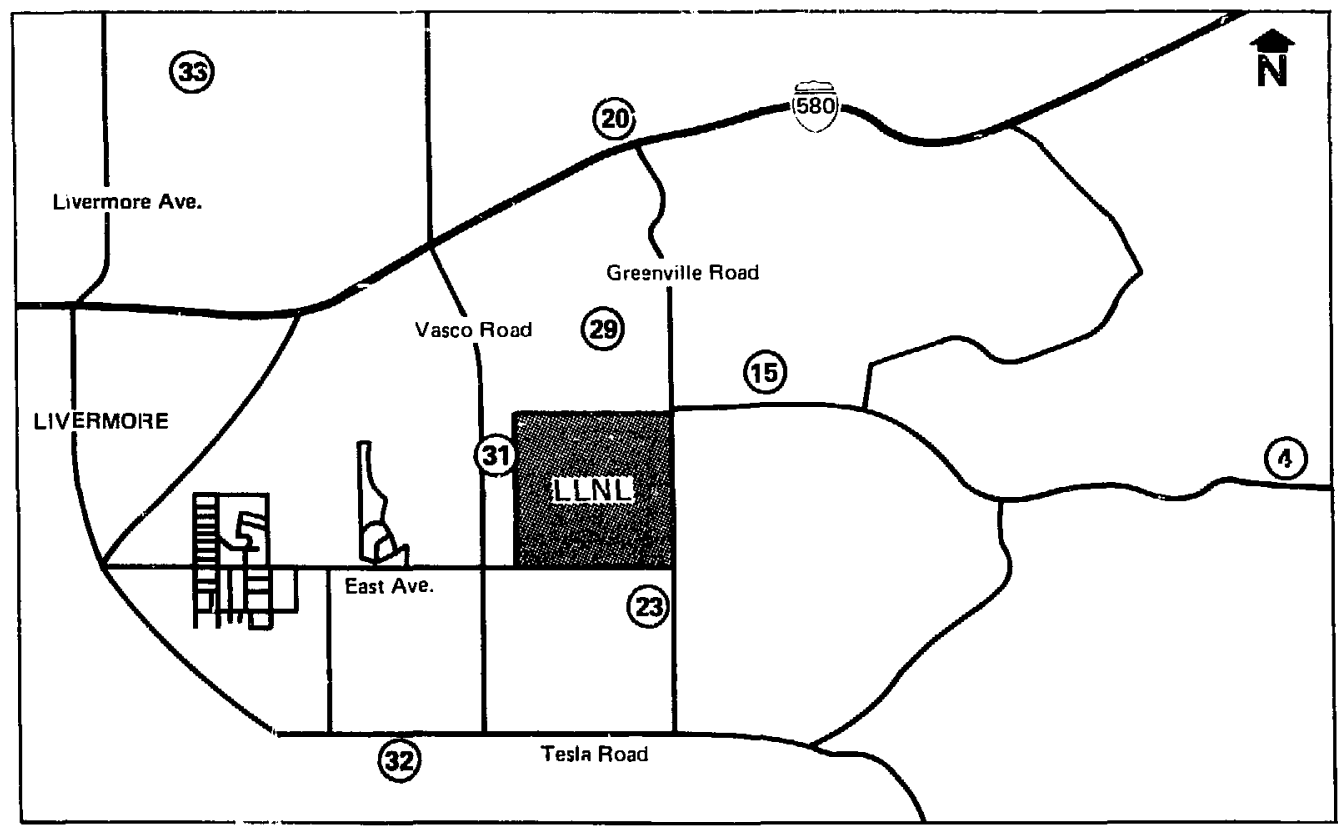

Figure 12. Livermore Valley vegetation-sampling locations. 


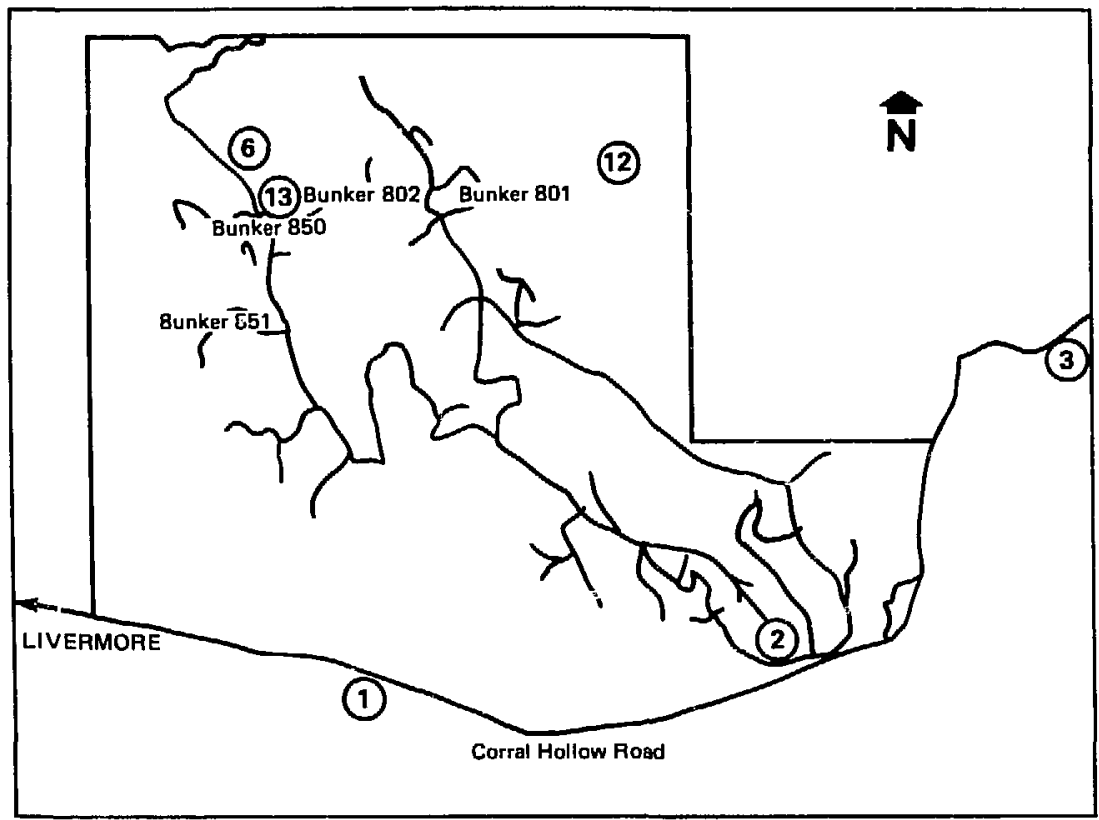

Figure 13. Site 300 vegetation-sampling locations.

was determined by gas proportional counting. The data in Table 24 show that the tritium content of Livermore Valley honey samples is comparable to that of honey from neighboring areas. Because of the dry 1980 fall season fewer varieties of honey were available in 1981.

\section{Radioactivity in Milk}

During 1981, goat-milk samples were obtained from three farms within about $5 \mathrm{~km}$ of the Laboratory. A portion of each sample was vacuum-distilled and the distillate analyzed for tritium activity by liquid-scintillation counting. Another portion was oven-dried, and the residue blended and gamma counted in a $\mathrm{Ge}(\mathrm{Li})$ system. The activities of the ${ }^{137} \mathrm{Cs}$, ${ }^{40} \mathrm{~K}$, and ${ }^{3} \mathrm{H}$ in the samples are shown in Table 25 . The annual average concentration of tritium in milk, $7.9 \times 10^{-7}$ $\mu \mathrm{Ci} / \mathrm{ml}$, is greater than reported in 1980 because of an elevated December value $\left(111 \times 10^{-7} \mu \mathrm{Ci} / \mathrm{ml}\right)$. An increase in HTO released from the tritium facility during December probably was responsible for this increase. Also chown are the calculated annual whole-body or critical-organ radiation doses that could be received from consuming this milk. These calculations are based on a milk intake of $\mathbf{3 1 0}$ liters/y and on the models previously referenced. The only dose above $1 \mathrm{mrem}$ to an individual is from naturally occurring ${ }^{40} \mathrm{~K}$.

\section{Environmental Radiation Measurements}

Quarterly, environmental radiation is measured at the 22 LL.NL perimeter locations (Fig. 14) and at the 47 off-site locations (Fig. 15). These measurements are obtained with thermoluminescence dosimeters using a previously published procedure. ${ }^{10}$ Based on past measurements, ${ }^{11}$ environmental terrestrial exposure rates in the Livermore Valley vary from 30 to $60 \mathrm{mR} / \mathrm{y}$. Cosmic radiation, calculated from the local elevation and geomagnetic latitude according to the data of Lowder and Beck, ${ }^{12}$ is approximately $35 \mathrm{mR} / \mathrm{y}$.

Table 26 shows quarterly and annual radiation doses (in millirem) derived from measurements at perimeter locations. The operation of a $14-\mathrm{MeV}$ neutron generator adjacent to the south boundary fence was responsible for the elevated doses at Location 5 on the south site boundary directly opposite this facility. However, these doses are lower than those observed during 1980 


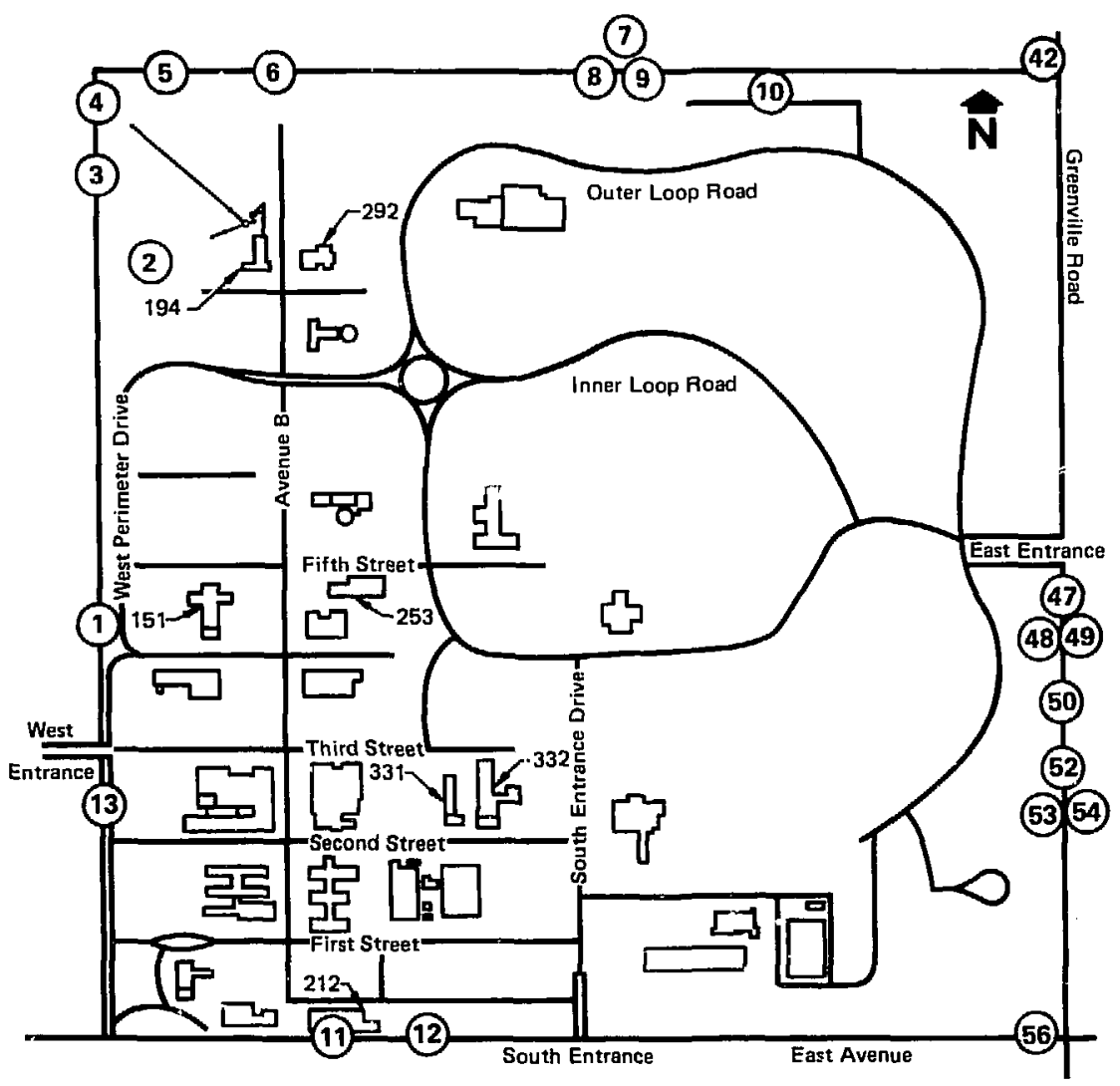

Figure 14. LLNL perimeter locations for gamma dosimeters.

because many high-flux experiments normally performed at this location are now being performed in Bldg. 292 in the northwest quadrant of the Laboratory.

The median annual off-site dose of 57 mrem is less than that observed in 1980. Although the median annual perimeter dose of 54 mrem is slightly lower than the 63 mrem of 1980 , this difference is not considered statistically significant. Perimeter locations 3, 4, and 5 are near a linear accelerator facility. Figure 16 shows the 1981 annual frequency distribution of environmental dose rates observed at the 47 off-site locations. The dosimeter that recorded the highest dose (132 mrem) was neai an off-site industrial plant where radiography is frequently performed. The second highest off-site exposure, 65 mrem over three quarters (first-quarter dosimeter packet was missing) was due to a 35-mrem third-quarter response from a dosimeter located in a remote area about $4 \mathrm{~km}$ northeast of LLNL. Dosimeter packets located close to LLNL operations showed no abnormal responses during that quarter. Figure 17 is a dose-distribution plot combining Laboratory perimeter and off-site measurements.

Environmental neutron dose rate measurements using ${ }^{235} U$ track-etch detectors are also made at eight locations on the LLNL site perimeter. A detailed description of the detector and the spark-counting procedure has been published. ${ }^{13}$ Neutron-monitoring locations are shown in Fig. 3 and 1981 quarterly measurements are shown in Table 27. Except for the first quarter of location 3 which monitors the $14-\mathrm{MeV}$ neutron generator in Bldg. 212, all levels are within the range typical of natural background. 


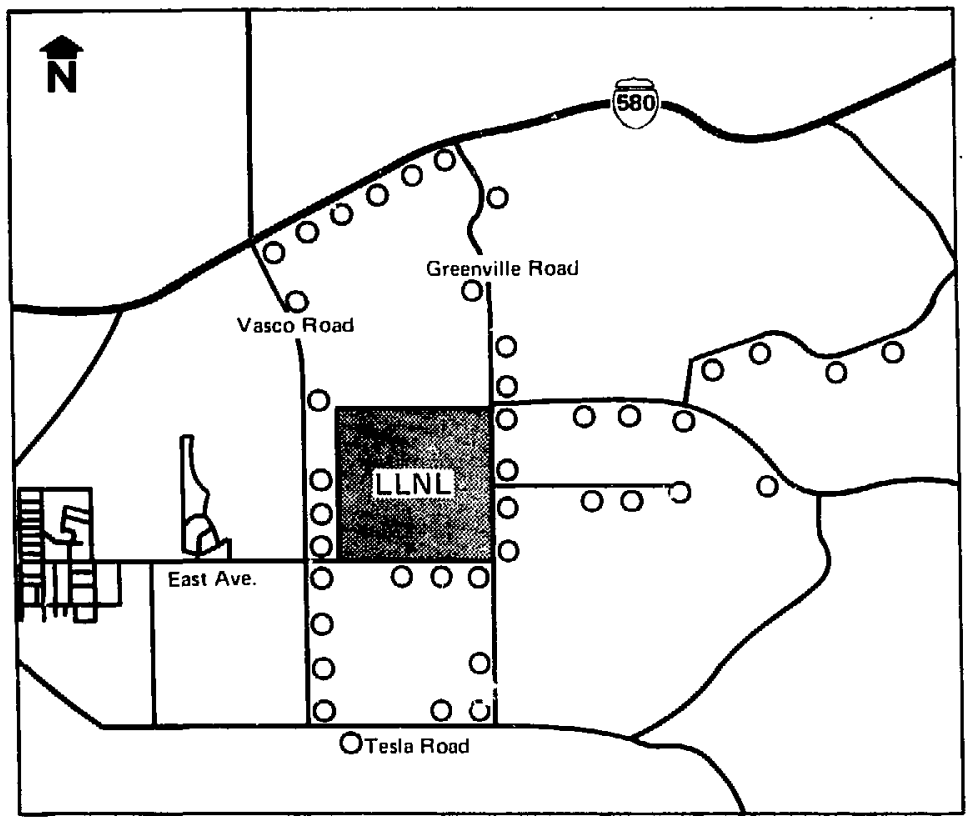

Figure 15. LLNL off-site locations for gamma dosimeters.

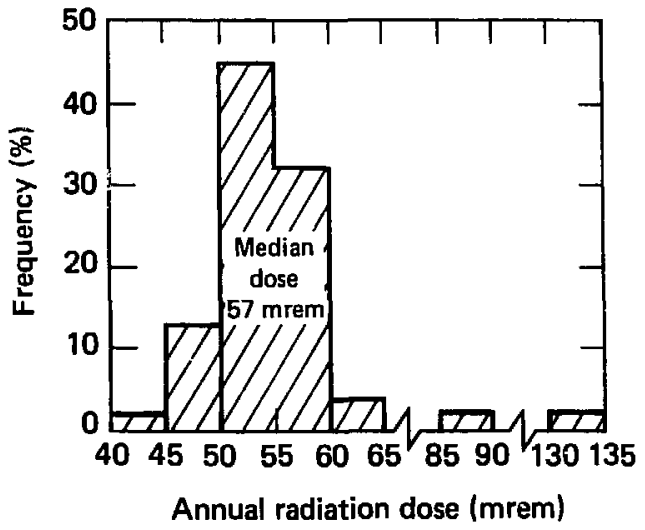

Figure 16. Annual off-site gamma radiation background for 1981.

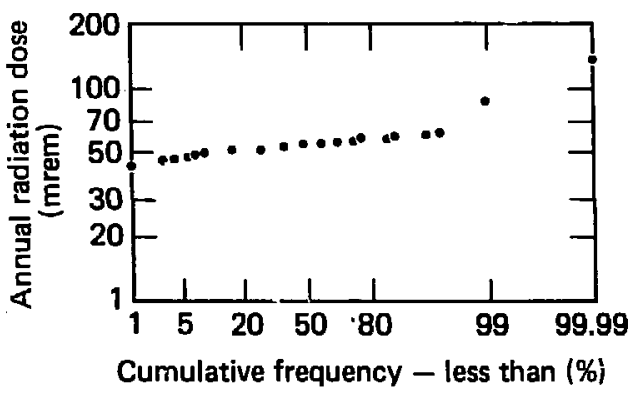

Figure 17. TLD dose-distribution plot for the LLNL perimeter and Livermore Valley during 1981. 


\section{Nonradioactive Monitoring}

\section{Beryllium in Air and Water}

Beryllium monitoring of air both on-site and at or near LLNL property boundaries has always been a part of the Laboratory's safety program. Monthly, half of every filter from the LLNL perimeter and Site 300 is composited by sampling location and wet-digested. Then the beryllium content of the solutions is determined by atomic absorption analysis.

Tables 28 and 29 show average monthly concentrations of airborne beryllium for LLNL perimeter and Site 300 sampling locations, respectively. There appear to be no differences between the levels at Site 300 , where beryllium is frequently expended in high-explosive experiments, and those observed at Livermore. The concentrations, which are two to three orders of magnitude below the emission standard, can be accounted for by resuspension of surface soil containing naturally occurring beryllium. Local soils contain approximately $1 \mathrm{ppm}$ of beryllium, and Livermore's air typically contains $10-100 \mu \mathrm{g}$ of particulates per cubic meter. By using a ralue of $50 \mu \mathrm{g} / \mathrm{m}^{3}$ for an average dust load and $1 \mathrm{ppm}$ for beryllium content of this dust, an airborne beryllium concentration of $5.0 \times 10^{-5} \mu \mathrm{g} / \mathrm{m}^{3}$ can be calculated. This value is in good agreement with the data in Tables 28 and 29. Average annual concentrations are less than $3 \%$ of the appropriate standard.

In compliance with the California Regional Water Quality Control Board (WQCB) Order 80-184, we began quarterly sampling of Site 300 water-monitoring locations for beryllium (in addition to gross alpha, gross beta, and tritium), as well as surface water runoff at disposal pits during the rainy season.

No beryllium was detected in any of the samples (limit of detection is $10 \mu \mathrm{g} / \mathrm{liter}$ ). Alpha and beta activities were also below the limit of sensitivity.

\section{Nonradioactive Pollutants in Sewage}

As noted previously, sanitary sewage from the Laboratory is treated at the LWRP. This effluent is continuously monitored for $\mathrm{pH}$ and radioactivity before it enters the Livermore sewer system. $^{14}$ Sewage samples representative of daily flow are collected and composited monthly, and the composites are analyzed for the metals shown in Table 30.

In addition, samples of Laboratory sewage effluent are collected quarterly. These samples are analyzed for the parameters specified on LWRP's permit (National Permit Discharge Elimination System). Table 31 shows the data for 1981. All data demonstrate compliance with the City of Livermore's discharge limits listed in Appendix D.

\section{Noise Pollution}

As noted elsewhere, the Laboratory's high-explosive diagnostic work is conducted at Site $\mathbf{3 0 0}$. Because Site $\mathbf{3 0 0}$ is so remote, these experiments can be performed with minimal off-site impact from annoying noises or damaging overpressures. On the basis of meteorological measurements made twice each day, a limit is set on the weight of high explosives that can be detonated without impact in populated areas. To monitor these limits, four microbarograph sensors are maintained in or near the city of Tracy. The probability of overpressure is greatest in the Tracy area because of the direction of the prevailing winds. There were no complaints of overpressures attrib. uted to LLNL operations at Site 300 during 1981.

\section{Pollutants in Storm Runoff and Liquid- Discharge Sites}

Beginning in 1975, the Laboratory's environmental surveillance program was expanded to :clude pesticide monitoring. Pesticides usec at LLNL include nerivicides, furgicides, and insecticides. The most probable way pesticides used at LLNL could be transported to the off-site environment is by entrainment in surface runoff water. Most of this surface drainage leaves the Laboratory via a ditch at the northwest corner of the property. A sample was collected from the ditch following the first major storm.

This sample was extracted with organic solvents, and the extracts were analyzed by gas-liquid chromatography (GLC) using a variety of detectors. Data obtained from these analyses were compared with the pesticides listed in Title 22. of the California Health and Safety Code. ${ }^{7}$ No materials were detected in the samples at concentrations exceeding the State adopted standards for these organic chemicals.

Order 80-183 from the Water Quality Control Board requires the monthly sampling and analyses of wells which are down gradient from Site 300 liquid-discharge sites (locations 3, 4, 5, and 14). Any increase in total organic carbon, specific conductance, chlorides, or total hardness could indicate percolation of the liquid waste. The data shown in Table 32 do not indicate contamination. 


\section{Environmental Impact of LENL Operations}

\section{Radioactive Airborne Effluents}

In 1981 (see Table 33), radioactive airborne effluents consisted of a total of $2620 \mathrm{Ci}$ of tritiurn from Bldg. 212 (14-MeV neutron generator), Bldg. 331 (tritium facility), and Bldg. 292 (rotating target neutron source), and $344 \mathrm{Ci}$ of ${ }^{15} \mathrm{O}_{2}-{ }^{13} \mathrm{~N}_{2}$ from Bldg. 194 (electron-positron linear accelerator). All radionuclides, with the exception of tritium, are short-lived. Comparative releases of radioactive effluents at Livermore during the 6-year period 1976 through 1981 are shown in Table 34. The Livermore reactor was shut down on March 31, 1980, because of a lack of programmatic need. Closure of this facility removes the local source of ${ }^{41} \mathrm{Ar}$ and eliminates a major contributor to the site radiation dose to the public.

Table 33 also contains estimated radiation doses to the public from these radioactive airborne effluents. Three dose-reference points were used: (1) the "fence-post" dose at that location on the site boundary where maximum exposure rates exist, (2) the close to the nearest resident, and (3) the man-rem dose within a radius of $80 \mathrm{~km}$.

Dose calculations were made using a continuous-point-source computer code based on the Gaussian plume model. ${ }^{15}$ This code provides ratios of concentration to release rate $(x / Q)$ through sixteen, $22.5^{\circ}$ compass sectors, and distances from 0.1 to $100 \mathrm{~km}$ from potential release points. The average annual $x / Q$ values have been calculated using local meteorological data from an instrumented tower. This tower, located near the Laboratory's east site boundary, is equipped with sensors mounted at 10 - and $40-\mathrm{m}$ levels that measure wind direction, wind speed, and temperature. From records of these data, wind speed, wind direction, and atmospheric stability estimates were tabulated at $1 / 4-\mathrm{h}$ intervals over the calendar year. Variance in the horizontal wind direction was used to estimate Pasquill-Gifford stability categories based on the method described by Slade. ${ }^{16}$ Lateral and vertical standard deviations, $\sigma_{y}$ and $\sigma_{z}$, are entered in the computer code as functions of these stability categories and the respective distances. From annual effluent data the release rate $Q$ (in curies per second) was calculated for each of the principal radionuclides released to the atmosphere, and the concentrations at the site boundary and the nearest resident were calculated from appropriate $\chi / Q$ values. "Nearest resident" means that resident receiving the highest dose from each radioactivity release point, not necessarily the resident nearest to the site boundary. Dose estimates were based on the dose-conversion factor in the NRC Regulatory Guide $1.109 .{ }^{9}$ The results indicate that the maximum estimated dose to the nearest resident was less than 1 mrem.

Table 33 shows a combined population dose of less than 2 man-rem from ${ }^{3} \mathrm{H}$ and neutron activation products in Laboratory airborne effluents. This dose is based on a population of $4.8 \times 10^{6}$ within $80 \mathrm{~km}$ of the Laboratory. Using 100 $\mathrm{mrem} / \mathrm{y}$ as a typical average radiation dose from natural sources, the comparable natural radiation dose the same group receives is $4.8 \times 10^{5}$ man-rem. By comparison, the population dose from Laboratory operations is negligible.

\section{Radioactive Liquid Effluents}

Low-level radioactive wastewater is treated to reduce radioactivity to levels as low as reasonably achievable and well within applicable health and safety standards. The treated effluent is then discharged into Livermore's sanitary sewer system. During 1981, the principal radionuclides released into the sewer system were $0.92 \times 10^{-4} \mathrm{Ci}$ of ${ }^{239} \mathrm{Pu}$ and $6 \mathrm{Ci}$ of HTO. Table 13 shows that the average annual concentrations of these radionucìdes represent $2.0 \times 10^{-4} \%$ and $1.0 \times 10^{-2} \%$ of the DOE standards.

\section{Nonradioactive Liquid Effluents}

The metals in sewage are listed in Table 30. The quantities of metals discharged did not prevent the LWRP from discharging effluents within limits set by the WQCB.

The first rains of the season, which were collected as storm drain runoff, showed no detectable level of specified total identifiable chlorinated hydrocarbons, and the concentration of substances in the runoff was generally lower than in the influent. These data are displayed in Table 35.

\section{Quality Assurance}

During 1981, the Laboratory participated in the Environmental Protection Agency's interlaboratory Cross Check program and DOE's Sample Analysis Intercomparison program conducted for DOE by the Environmental Measurements Laboratory (EML).

A statistical summary is published annually by EML comparing the analytical performance of participating DOE contractors with measurements made by EML. ${ }^{17,18}$ Table 36 shows the comparison 
of analyses from LINL with EML. The analytical results are compared by the ratio of LLNL data to that of EML and the ratio of LLNL data to the respective mean of all analyses reported.
A description of the sampling and analytical procedures used at LLNL has been included as Appendix E. 


\section{References}

1. C. L. Lindeken, R. L. Morgan, and K. F. Petrock, "Collection Efficiency of Whatman 41 Filter Paper for Submicron Aerosols," Health Phys. 9, 305 (1963).

2. A. J. Toy, C. L. Lindeken, K. S. Griggs, and R. W. Buddemeier, Environmental Monitoring at the Lawrence Livermore National Laboratory, 1980 Annual Report, Lawrence Livermore National Laboratory, Livermore, CA, UCRL-50027-80 (1981).

3. P. H. Gudiksen, C. L. Lindeken, J. W. Meadows, and K. O. Hamby, Environmental Levels of Radioactivity in the Vicinity of the LLL, 1972 Annual Report, Lawrence Livermore National Laboratory, Livermore, CA, UCRL-51333 (1973).

4. W. J. Tipton, An Aerial Radiological Survey of the Lawrence Lizermore Laboratory, EG\&G, Las Vegas, NV, EGG-1183-1693 (1975).

5. R. W. Buddemeier, Tritium-Salinity Relationships in San Francisco Bay, Lawrence Livermore National Laboratory, Livermore, CA, UCRL-86669 (1981).

6. National Interim Primary Drinking Water Regulations, Environmental Protection Agency, EPA-570/9-76-003 (June 24, 1977).

7. California Domestic Water Quality and Monitoring Regulations, California Administrative Title Code 22, State of California (1977).

8. W. J. Silver, C. L. Lindeken, K. M. Wong, E. H. Willes, and J. H. White, Environmental Monitoring at the Lawrence Livermore Laboratory, 1977 Annual Report, Lawrence Livermore National Laboratory, Livermore, CA, UCRL-50027-77 (1978).

9. Calculation of Annual Doses to Man from Routine Releases of Reactor Effluent for the Purpose of Evaluating Compliance with 10CFR Pait 50, Appendix 1, U. S. Nuclear Regulatory Commission, Regulatory Guide 1.109 (October 1977).

10. C. L. Lindeken, J. H. White, A. J. Toy, and C. W. Sundbeck, Ambient Environmental Radiation Monitoring at the Lawrence Livermore National Laboratory, Lawrence Livermore National Laboratory, Livermore, CA, UCRL-77106 (1975).

11. C. L. Lindeken, P. H. Gudiksen, J. W. Meadows, K. O. Hamby, and L. R. Anspaugh, Environmental Levels of Radioactivity in Livermore Valley Soils, Lawrence Livermore National Laboratory, Livermore, CA, UCRL-74414 (1973).

12. W. M. Lowder and H. L. Beck, "Cosmic Ray Ionization in the Lower Atmosphere," J. Geo. Phys. Rev. 71, 4611 (1966).

13. J. C. Fisher, Jr., "Calibration of Anderson-Braun Remmeters with Track Etch Detectors," in Hazards Control Progress Report Nc. 52, Lawrence Livermore National Laboratory, Livermore, CA, UCRL-50007-76-1 (1976), p. 37.

14. M. Auyong, J. L. Cate, Jr., and D. W. Rueppel, On-Line Monitoring of Toxic Materials in Sewage at the Lawrence Livermore Laboratory, Lawrence Livermore National Laboratory, Livermore, CA, UCRL-83664 (1980).

15. K. R. Peterson, T. W. Crawford, and L. A. Lawson, CPS: A Continuous-Point-Source Computer Code for Plume Dispersion and Deposition Calculations, Lawrence Livermore National Laboratory, Livermore, CA, UCRL-52049 (1976).

16. D. H. Slade, Ed., Meteorology and Atomic Energy (U. S. Atomic Energy Commission, 1968).

17. C. G. Sanderson, Report of the Department of Energy, Division of Operational and Environmental Safety-Quality Assurance Program, Environmental Measurements Laboratory, New York, NY, EML-393 (August 3, 1981).

18. C. G. Sanderson, Quarterly Report of the Department of Energy, Division of Operational and Environmental Safety. Quality Assessment Program, Environmental Measurements Laboratory, New York, NY, EML-402 (February 1, 1982). 


\section{Appendix A. Tables}

The statistical values ( $2 \sigma$ estimates) that accompany individual measurements of radioactivity in the following tables are the result of counting statistics. The minimum detection limit is assumed reached when the $2 \sigma$ estimate is $\pm 100 \%$. Statistical values for groups of data like annual averages are calculated standard deviations of the mean (average). Standard deviations of the mean are reported at the $95 \%$ confidence level.

Table 1. Gross alpha activity on air filters - LLNL perimeter and Livermore Valley.

\begin{tabular}{|c|c|c|c|c|c|c|c|c|c|c|c|c|c|c|c|}
\hline \multirow[b]{2}{*}{ Location" } & \multicolumn{13}{|c|}{$10^{-15} \mu \mathrm{Ci} / \mathrm{ml}$} & \multirow[b]{2}{*}{$\%$ SDM $^{\mathrm{b}}$} & \multirow[b]{2}{*}{$\% \mathrm{CG}^{\mathrm{C}}$} \\
\hline & Jan. & Feb. & Mar. & Apr. & May & June & July & Aug. & Sept. & Oct. & Nov. & Dec. & Average & & \\
\hline \multicolumn{16}{|l|}{ Perimeter } \\
\hline 01 & 1.1 & 0.4 & 0.5 & 0.6 & 0.5 & 0.9 & 0.8 & 0.9 & 0.6 & 1.8 & 1.1 & 0.8 & 0.8 & 46 & 4 \\
\hline 02 & 1.5 & 0.4 & 0.4 & 0.5 & 0.5 & 0.9 & 1.0 & 0.8 & 0.8 & 0.9 & 0.8 & 0.7 & 0.8 & 40 & 4 \\
\hline 12 & 0.6 & 0.6 & 0.4 & 0.4 & 0.5 & 1.3 & 0.9 & 0.8 & 0.7 & 0.8 & 0.7 & 0.8 & 0.7 & 35 & 4 \\
\hline 13 & 0.7 & 0.4 & 0.4 & 0.4 & 0.4 & 0.5 & 0.5 & 0.4 & 0.4 & 0.6 & 0.4 & 0.4 & 0.5 & 22 & 2 \\
\hline 14 & 0.9 & 0.5 & 0.6 & 0.6 & 0.5 & 0.6 & 0.6 & 0.5 & 0.5 & 9.7 & 0.8 & 0.7 & 0.6 & 21 & 3 \\
\hline 15 & 0.7 & 0.5 & 0.4 & 0.5 & 0.5 & 0.5 & 0.7 & 0.4 & 0.4 & 0.6 & 0.4 & 0.4 & 0.5 & 22 & 2 \\
\hline \multicolumn{16}{|l|}{ Valley } \\
\hline 03 & 1.0 & 0.7 & 0.7 & 0.7 & 0.7 & 0.8 & 0.8 & $0 . \beta$ & 0.6 & 0.7 & 0.7 & 0.7 & 0.7 & 13 & 4 \\
\hline 04 & 1.6 & 0.9 & 0.8 & 0.8 & 0.9 & 0.9 & 0.6 & 0.5 & 0.5 & 0.6 & 0.5 & 0.5 & 0.8 & 41 & 4 \\
\hline 05 & 1.1 & 0.7 & 0.7 & 0.7 & 0.7 & 0.8 & 0.8 & 0.7 & 0.7 & 0.9 & 0.7 & 0.6 & 0.8 & 17 & 4 \\
\hline 06 & 0.9 & 0.9 & 0.7 & 0.7 & 0.8 & 0.9 & 0.8 & 0.7 & 0.7 & 0.6 & 0.7 & 0.7 & 0.8 & 13 & 4 \\
\hline 07 & lost & lost & 0.7 & 0.8 & 0.8 & 0.8 & 0.9 & 0.7 & 0.8 & 0.9 & 0.7 & 0.6 & 0.8 & 12 & 4 \\
\hline 08 & 2.0 & 0.9 & 1.0 & 1.0 & 0.9 & 0.9 & 1.0 & 0.8 & 0.7 & 0.7 & 0.8 & 0.8 & 1.0 & 35 & 5 \\
\hline 09 & 1.4 & 1.2 & 0.7 & 0.8 & 0.9 & 1.2 & 0.9 & 0.7 & 0.7 & 1.9 & 0.8 & 0.9 & 1.0 & 36 & 5 \\
\hline 10 & 0.8 & 0.6 & 0.6 & 0.6 & 0.4 & 0.9 & 0.9 & 0.8 & 0.8 & 0.9 & 0.9 & 0.8 & 0.8 & 21 & 4 \\
\hline 11 & 1.5 & 0.9 & 0.7 & 0.7 & 0.8 & 0.8 & 1.2 & 0.8 & 0.7 & 0.7 & 0.7 & 0.8 & 0.9 & 29 & 4 \\
\hline 16 & 0.7 & 0.5 & 0.7 & 0.7 & 0.6 & 0.7 & 0.7 & 0.5 & 0.7 & 0.9 & 0.7 & 0.8 & 0.7 & 16 & 4 \\
\hline 17 & 1.8 & 1.1 & 0.7 & 0.9 & 0.8 & 0.8 & 1.5 & 1.5 & 1.2 & 0.8 & 0.8 & 0.7 & 1.1 & 35 & 6 \\
\hline Annual average & & & & & & & & & & & & & 0.8 & $\mathbf{2 0}$ & 4 \\
\hline
\end{tabular}

"See Figs, 3 and 5.

${ }^{6} \% \mathrm{SDM}=\%$ standard deviation of mean at $1 \sigma$.

concentration guide $(\mathrm{CG})=2.0 \times 10^{-14} \mu \mathrm{Ci} / \mathrm{ml}$. 
Table 2. Gross beta activity on air filters - LLNL perimeter and Livermore Valley.

$10^{-14} \mu \mathrm{Ci} / \mathrm{ml}$

Location" Jan. Feb. Mar. Apr. May Jure July Aug. Sept. Oct. Nov. Dec. Average \% SDM \% CG'

\section{Perimeter}

\begin{tabular}{|c|c|c|c|c|c|c|c|c|c|c|c|c|c|c|c|}
\hline 01 & 17.8 & 11.0 & 17.3 & 20.5 & 15.7 & 13.0 & 11.2 & 4.3 & 3.0 & 6.6 & 1.5 & 1.3 & 10.3 & 66 & 10 \\
\hline 02 & 21.0 & 16.2 & 17.1 & 21.4 & 18.5 & 16.7 & 12.3 & 3.5 & 3.1 & 3.2 & 1.9 & 1.2 & 11.3 & 71 & 11 \\
\hline 12 & 9.2 & $\epsilon .0$ & 14.5 & 17.1 & 13.2 & 13.1 & 9.8 & 3.2 & 2.1 & 2.4 & 1.4 & 0.9 & 7.7 & 75 & 8 \\
\hline 13 & 12.7 & 13.2 & 13.1 & 17.3 & 16.3 & 10.8 & 9.1 & 2.9 & 2.4 & 2.8 & 1.8 & 1.0 & 8.6 & 71 & 9 \\
\hline 14 & 16.5 & 16.7 & 17.5 & 20.9 & 17.8 & 13.8 & 11.3 & 3.6 & 3.3 & 3.5 & 1.7 & 1.2 & 10.7 & 70 & 11 \\
\hline 15 & 12.4 & 16.8 & 17.0 & 17.0 & 18.9 & 10.8 & 9.4 & 28 & 2.6 & 2.5 & 1.6 & 1.1 & 9.4 & 74 & 9 \\
\hline D3 & 10.6 & 11.5 & 9.4 & 8.8 & 8.9 & 8.6 & 6.4 & 3.5 & 2.5 & 3.3 & 2.4 & 2.1 & 6.5 & 54 & 6 \\
\hline 04 & 26.7 & 19.6 & 18.6 & 23.1 & 23.4 & 15.4 & 9.2 & 3.4 & 2.9 & 2.7 & 1.8 & 1.3 & 12.3 & 79 & 12 \\
\hline 05 & 9.2 & 6.6 & 6.2 & 8.0 & 8.5 & 8.4 & 9.0 & 4.4 & 3,5 & 3.1 & 26 & 2.2 & 6.0 & 45 & 6 \\
\hline 06 & 11.2 & 12.9 & 11.8 & 13.1 & 11.5 & 8.6 & 7.0 & 3.7 & 3.1 & 3.1 & 2.4 & 2.2 & 7.6 & 59 & 8 \\
\hline 07 & lost & lost & 11.0 & 14.7 & 13.0 & 11.3 & 8.7 & 4.4 & 3.6 & 3.3 & 1.9 & 2.2 & 7.4 & 65 & 7 \\
\hline 08 & 19.2 & 17.3 & 14.4 & 17.0 & 14.9 & 12.2 & 9.8 & 3.3 & 2.6 & 3.2 & 2.0 & 1.4 & 9.8 & 70 & 10 \\
\hline 09 & 14.3 & 20.5 & 18.9 & 21.5 & 17.7 & 10.9 & 10.9 & 4.7 & 4.3 & 5.0 & 3.4 & 2.9 & 11.2 & 64 & 11 \\
\hline 10 & 7.9 & 12.3 & 11.1 & 12.3 & 2.3 & 11.9 & 10.0 & 3.8 & 2.9 & 3.0 & 1.6 & 1.1 & 6.7 & 69 & 7 \\
\hline 11 & 11.8 & 19.6 & 16.9 & 15.3 & 15.3 & 12.3 & 9.0 & 4.7 & 3.9 & 4.2 & 2.8 & 2.2 & 9.8 & 63 & 10 \\
\hline 16 & 9.2 & 14.2 & 12.6 & 19.9 & 16.6 & 14.3 & 8.7 & 2.8 & 2.7 & 2.7 & 2.0 & 1.5 & 8.9 & 73 & 9 \\
\hline 17 & 25.0 & 20.8 & 15.7 & 23.0 & 181.8 & 14.7 & 124 & 7.4 & 4.8 & 2.6 & 2.0 & 1.1 & 12.4 & 70 & 12 \\
\hline al av & & & & & & & & & & & & & 9.2 & 22 & 9 \\
\hline
\end{tabular}

'See Figs. 3 and 5.

${ }^{b} \mathrm{CG}=1.0 \times 10^{-12} \mu \mathrm{Ci} / \mathrm{ml}$.

Table 3. Gross alpha activity on air filters - Site 300 .

\begin{tabular}{|c|c|c|c|c|c|c|c|c|c|c|c|c|c|c|c|}
\hline \multirow[b]{2}{*}{ Location ${ }^{2}$} & \multicolumn{13}{|c|}{$10^{-15} \mu \mathrm{Ci} / \mathrm{ml}$} & \multirow[b]{2}{*}{$\%$ SDM } & \multirow[b]{2}{*}{$\% \mathrm{CG}^{\mathrm{b}}$} \\
\hline & Jan. & Feb. & Mar. & Apr. & May & June & July & Aug. & Sept. & Oct. & Mov. & Dec. & Average & & \\
\hline 01 & 0.9 & 0.6 & 0.4 & 0.6 & 0.5 & 0.6 & 0.5 & 0.6 & 0.6 & 0.7 & 0.4 & 0.3 & 0.6 & 29 & 3 \\
\hline 02 & lost & 0.4 & 0.4 & 0.4 & 0.5 & 0.5 & 0.5 & 0.6 & 0.5 & 0.7 & 0.3 & 0.4 & 0.5 & 23 & 2 \\
\hline 03 & 1.0 & 0.6 & 0.5 & 0.6 & 0.7 & 0.6 & 0.6 & 0.6 & 0.6 & 0.6 & 0.5 & 0.5 & 0.6 & 21 & 3 \\
\hline 04 & 1.7 & 0.6 & 0.6 & 0.6 & lost & 0.6 & 0.6 & 0.5 & 0.5 & 0.6 & 0.4 & 0.4 & 0.6 & 56 & 3 \\
\hline 05 & 0.8 & 0.4 & 0.4 & 0.5 & 0.6 & 0.6 & 0.6 & 0.5 & 0.5 & 0.6 & 0.3 & 0.4 & 0.5 & 25 & 2 \\
\hline 06 & 6.3 & 0.9 & 1.0 & 0.9 & 0.8 & 1.1 & 1.0 & 0.8 & 0.7 & 1.6 & 0.8 & 0.9 & 1.4 & 111 & 7 \\
\hline 08 & 1.1 & 0.4 & 0.4 & 0.5 & 0.4 & 0.5 & 0.5 & 0.4 & 0.5 & 0.5 & 0.5 & 0.4 & 0.5 & 37 & 2 \\
\hline 09 & 0.9 & 0.4 & 0.4 & 0.6 & 0.5 & 0.5 & 0.4 & 0.5 & 0.5 & 0.6 & 0.4 & 0.6 & 0.5 & 27 & 2 \\
\hline 10 & 1.2 & 0.5 & 0.5 & 0.6 & 0.5 & 0.5 & 0.7 & 0.5 & 0.5 & 0.8 & 0.5 & 0.5 & 0.6 & 35 & 3 \\
\hline Annual average & & & & & & & & & & & & & 0.6 & 45 & 3 \\
\hline
\end{tabular}

'See Fig. 4. Location 6 is in city of Tracy.

${ }^{\mathrm{b}} \mathrm{CG}=2.0 \times 10^{-14} \mu \mathrm{Ci} / \mathrm{ml}$. 
Table 4. Gross beta activity on air filters - Site 300 .

\begin{tabular}{|c|c|c|c|c|c|c|c|c|c|c|c|c|c|c|c|}
\hline \multirow[b]{2}{*}{ Location" } & \multicolumn{13}{|c|}{$10^{-14} \mu \mathrm{Cj} / \mathrm{ml}$} & \multirow[b]{2}{*}{$\%$ SDM } & \multirow[b]{2}{*}{$\% \mathrm{CG}^{\mathrm{b}}$} \\
\hline & Jan. & Feb. & Mar. & Apr. & May & June & July & Aug. & Sept. & Oct. & Nov. & Dec. & Average & & \\
\hline 01 & 18.8 & 16.4 & 16,9 & 25.0 & 20.7 & 19.3 & 14.0 & 7.0 & 3.4 & 3.2 & 2.1 & 1.5 & 12.4 & 68 & 12 \\
\hline 02 & lost & 16.1 & 17.6 & 23.8 & 27.8 & 19.6 & 13.8 & 7.2 & 3.7 & 3.3 & 2.2 & 1.5 & 124 & 75 & 12 \\
\hline 03 & 20.1 & 18.9 & 19.9 & 24.6 & 21.1 & 19.6 & 14.8 & 6.8 & 3.3 & 3.2 & 2.5 & 1.9 & 13.1 & 67 & 13 \\
\hline 04 & 22.3 & 15.2 & 13.3 & 19.8 & lost & 14.5 & 12.1 & 5.4 & 2.9 & 2,6 & 1.9 & 1.3 & 10.1 & 75 & 10 \\
\hline 05 & 14.9 & 15.7 & 15.9 & 21.8 & 21.9 & 19.3 & 11.5 & 5.4 & 2.9 & 27 & 20 & 1.0 & 11.2 & 72 & 11 \\
\hline 06 & 21.7 & 18.1 & 17.5 & 26.5 & 23.1 & 15.3 & 10.9 & 4.9 & 1.5 & 5.2 & 2.5 & 2.1 & 124 & 73 & 12 \\
\hline 08 & 15.8 & 14.2 & 16.1 & 19.7 & 17.0 & 15.7 & 10.0 & 4.9 & 2.5 & 2.1 & 2.2 & 1.4 & 10.1 & 69 & 10 \\
\hline 09 & 16.3 & 19.0 & 16.0 & 25.5 & 22.3 & 18.2 & 13.1 & 6.9 & 3.3 & 3.0 & 23 & 1.5 & 12.3 & 69 & 12 \\
\hline 10 & 18.1 & 15.8 & 10.0 & 22.9 & 16.8 & 13.7 & 15.3 & 5.1 & 2.9 & 2.8 & 2.9 & 1.4 & 10.6 & 69 & 11 \\
\hline Annuai a7̌rage & & & & & & & & & & & & & 11.6 & 10 & 12 \\
\hline
\end{tabular}

'See Fig. 4. Location 6 is in city of Tracy.

${ }^{b} \mathrm{CG}=1.0 \times 10^{-12} \mu \mathrm{Ci} / \mathrm{ml}$.

Table 5. Gamma activity on air filters - LLNL perimeter.

\begin{tabular}{|c|c|c|c|c|c|c|}
\hline \multirow[b]{2}{*}{ Month } & \multirow{2}{*}{$\frac{10^{-13} \mu \mathrm{Ci} / \mathrm{ml}}{7 \mathrm{Be}} \pm 2 \sigma(\%)$} & \multicolumn{5}{|c|}{$10^{-16} \mu \mathrm{Ci} / \mathrm{ml} \pm 20(\%)$} \\
\hline & & ${ }^{80} \mathrm{~K}$ & ${ }^{106} \mathbf{R u}$ & ${ }^{125} \mathrm{Sb}$ & ${ }^{137} \mathrm{CB}$ & ${ }^{14} \mathrm{Ce}$ \\
\hline Jan. & $0.87 \pm 2$ & $11 \pm 35$ & $58 \pm 8$ & $6 \pm 2<$ & $7 \pm 8$ & $152 \pm 4$ \\
\hline Feb. & $1.01 \pm 3$ & $1.8 \pm 100$ & $103 \pm 7$ & $9.3 \pm 17$ & $13.1 \pm 6$ & $239 \pm 4$ \\
\hline Mar. & $0.94 \pm 2$ & $1.1 \pm 100$ & $150 \pm 3$ & $14.7 \pm 9$ & $19.9 \pm 4$ & $336 \pm 6$ \\
\hline Apr. & $1.01 \pm 2$ & $1.5 \pm 100$ & $190 \pm$ & $0.9 \pm 100$ & $26.3 \pm 4$ & $1.3 \pm 100$ \\
\hline May & $0.74 \pm 2$ & $5 \pm 49$ & $181 \pm 3$ & $17.9 \pm 7$ & $25.9 \pm 3$ & $387 \pm 2$ \\
\hline June & $0.89 \pm 2$ & $1.6 \pm 100$ & $150 \pm 4$ & $15.8 \pm 8$ & $24.6 \pm 3$ & $310 \pm 3$ \\
\hline July & $1.17 \pm 2$ & $10 \pm 39$ & $131 \pm 3$ & $13.5 \pm 9$ & $21.5 \pm 4$ & $243 \pm 3$ \\
\hline Aug. & $0.77 \pm 6$ & $6 \pm 100$ & $36 \pm 13$ & $4.3 \pm 25$ & $7.9 \pm 6$ & $75.8 \pm 7$ \\
\hline Sepl. & $1.1 \pm 2$ & $12 \pm 27$ & $30 \pm 15$ & $3.5 \pm 15$ & $6.5 \pm 6$ & $56 \pm 4$ \\
\hline Oct. & $1.3 \pm 2$ & $9.6 \pm 51$ & $14.9 \pm 11$ & $1.7 \pm 42$ & $3.8 \pm 8$ & $29.4 \pm 7$ \\
\hline Nov. & $0.6 \pm 4$ & $61 \pm 30$ & $5.7 \pm 64$ & $1.2 \pm 100$ & $1.6 \pm 22$ & $12.3 \pm 11$ \\
\hline Dec. & $0.5 \pm 3$ & $44 \pm 18$ & $29 \pm 100$ & $0.4 \pm 100$ & $1.3 \pm 32$ & $8.3 \pm 16$ \\
\hline Annual average & 0.91 & 18 & 84 & 7.4 & 13 & 154 \\
\hline$\%$ SDM & 26 & 126 & 81 & 87 & 74 & 92 \\
\hline $\mathrm{CG}(\mu \mathrm{Ci} / \mathrm{ml})$ & $4 \times 10^{-8}$ & $4 \times 10^{-9}$ & $2 \times 10^{-10}$ & $9 \times 10^{-10}$ & $5 \times 10^{-10}$ & $2 \times 10^{-10}$ \\
\hline$\% \mathrm{CG}$ & $2 \times 10^{-4}$ & $4 \times 10^{-5}$ & $4 \times 10^{-3}$ & $8 \times 10^{-5}$ & $3 \times 10^{-1}$ & $3 \times 10^{-3}$ \\
\hline
\end{tabular}


Table 6. Gamma activity on air filters - Site $\mathbf{3 0 0}$.

\begin{tabular}{|c|c|c|c|c|c|c|}
\hline \multirow[b]{2}{*}{ Month } & \multirow{2}{*}{$\frac{10^{-13} \mu \mathrm{Cl} / \mathrm{ml} \pm 2 \sigma(\%)}{{ }^{7} \mathrm{Bs}}$} & \multicolumn{5}{|c|}{$10^{-16} \mu \mathrm{Ci} / \mathrm{ml} \pm 2 \sigma(\%)$} \\
\hline & & ${ }^{{ }^{10} K} \mathbf{K}$ & ${ }^{106} \mathbf{R u}$ & ${ }^{12_{S b}}$ & ${ }^{137} \mathrm{Cs}$ & ${ }^{104} \mathrm{Ce}$ \\
\hline Jan. & $1.07 \pm 3$ & $27 \pm 67$ & $70 \pm 6$ & $6.1 \pm 15$ & $8.5 \pm 7$ & $178 \pm 5$ \\
\hline Feb. & $0.98 \pm 2$ & $1.0 \pm 100$ & $101 \pm 4$ & $9.3 \pm 14$ & $13.1 \pm 5$ & $231 \pm 7$ \\
\hline Mar. & $0.84 \pm 3$ & $0.7 \pm 100$ & $139 \pm 5$ & $12.7 \pm B$ & $18.3 \pm 3$ & $321 \pm 4$ \\
\hline Apr. & $0.006 \pm 100$ & $3.4 \pm 65$ & $248 \pm 4$ & $22.9 \pm 19$ & $34.0 \pm 5$ & $544 \pm 1.0$ \\
\hline May & $0.99 \pm 3$ & $3.4 \pm 58$ & $243 \pm 3$ & $23.5 \pm 6$ & $34.5 \pm 4$ & $541 \pm 2$ \\
\hline June & $1.25 \pm 2$ & $3.6 \pm 57$ & $247 \pm 2$ & $25.7 \pm 5$ & $38.1 \pm 3$ & $498 \pm 2$ \\
\hline July & $1.70 \pm 2$ & $6.0 \pm 47$ & $182 \pm 3$ & $19.7 \pm 6$ & $30.8 \pm 3$ & $353 \pm 2$ \\
\hline Aug. & $1.54 \pm 2$ & $3.4 \pm 49$ & $55 \pm 4$ & $7.0 \pm 8$ & $11.4 \pm 3$ & $107 \pm 3$ \\
\hline Sept. & $1.41 \pm 2$ & $1.3 \pm 100$ & $25 \pm 8$ & $3.7 \pm 13$ & $5.8 \pm 5$ & $52.4 \pm 4$ \\
\hline Oct. & $1.0 \pm 2$ & $3.3 \pm 71$ & $12 \pm 7$ & $1.7 \pm 15$ & $3.0 \pm 6$ & $23.4 \pm 6$ \\
\hline Nov. & $0.91 \pm 3$ & $1.0 \pm 100$ & $11 \pm 8$ & $1.3 \pm 30$ & $2.8 \pm 11$ & $20 \pm 9$ \\
\hline Dec. & $0.65 \pm 3$ & $25.5 \pm 23$ & $4.5 \pm 37$ & $1.0 \pm 65$ & $1.6 \pm 16$ & $9.1 \pm 15$ \\
\hline Annual average & 1.0 & 4.6 & 111 & 11 & 17 & 240 \\
\hline$\%$ SDM & 43 & 146 & 87 & 84 & 82 & 87 \\
\hline $\mathrm{CG}(\mu \mathrm{Ci} / \mathrm{ml})$ & $4 \times 10^{-8}$ & $4 \times 10^{-9}$ & $2 \times 10^{-10}$ & $9 \times 10^{-10}$ & $5 \times 10^{-10}$ & $2 \times 10^{-10}$ \\
\hline$\% \mathbf{C G}$ & $2 \times 10^{-4}$ & $1 \times 10^{-5}$ & $6 \times 10^{-3}$ & $1 \times 10^{-4}$ & $3 \times 10^{-1}$ & $1 \times 10^{-2}$ \\
\hline
\end{tabular}


Table 7. Plutonium-239 on air filten-selected locations.

\begin{tabular}{|c|c|c|c|c|c|c|c|c|c|c|c|c|c|c|c|}
\hline \multirow[b]{2}{*}{ Location" } & \multicolumn{13}{|c|}{$10^{-17} \mu \mathrm{Ci} / \mathrm{ml} \pm 2 \sigma(\%)$} & \multirow[b]{2}{*}{$\%$ SDM } & \multirow[b]{2}{*}{$\% C G^{b}$} \\
\hline & Jan. & Eeb. & Mar. & Apr. & May & June & July & Aug. & Sept. & Oct. & Nov. & Dec. & Average & & \\
\hline $090-04$ & $1.4 \pm 19$ & $1.8 \pm 13$ & $24 \pm 12$ & $3.4 \pm 13$ & $4.5 \pm 13$ & $11.8 \pm 8$ & $24 \pm 13$ & $1.0 \pm 15$ & $0.8 \pm 13$ & $0.4 \pm 72$ & $0.3 \pm 25$ & $0.2 \pm 26$ & 2.5 & 126 & $4 \times 10^{-2}$ \\
\hline $090-08$ & $0.8 \pm 25$ & $1.6 \pm 25$ & $2.1 \pm 15$ & $3.0 \pm 13$ & $27 \pm 16$ & $2.6 \pm 14$ & $2.2 \pm 15$ & $1.0 \pm 17$ & $0.5 \pm 16$ & $0.4 \pm 24$ & $0.3 \pm 39$ & $0.2 \pm 30$ & 1.4 & 32 & $2 \times 10^{-2}$ \\
\hline 090-10 & $0.8 \pm 19$ & $1.1 \pm 24$ & $1.5 \pm 15$ & $21 \pm 13$ & $0.4 \pm 50$ & Iosi & $2.8 \pm 13$ & $0.8 \pm 19$ & $0.7 \pm 19$ & $0.4 \pm 28$ & $0.3 \pm 41$ & $0.2 \pm 3.4$ & 1.0 & B1 & $2 \times 10^{-2}$ \\
\hline 090-16 & $0.8 \pm 29$ & $1.3 \pm 12$ & $1.5 \pm 12$ & $3.4 \pm 13$ & $3.7 \pm 11$ & $3.1 \pm 17$ & $2.5 \pm 13$ & $1.0 \pm 16$ & $0.8 \pm 19$ & $0.6 \pm 19$ & $0.4 \pm 25$ & $0.3 \pm 30$ & 1.6 & 76 & $3 \times 10^{-2}$ \\
\hline 090-17 & $1.0 \pm 22$ & $2.4 \pm 17$ & $2.1 \pm 13$ & $3.5 \pm 13$ & $3.3 \pm 11$ & $3.2 \pm 11$ & $3.3 \pm 24$ & $15 \pm 6$ & $0.7 \pm 19$ & $0.6 \pm 18$ & $0.3 \pm 43$ & $0.1 \pm 45$ & 3.0 & 135 & $5 \times 10^{-2}$ \\
\hline 040-06 & $1.0 \pm 16$ & $1.7 \pm 20$ & $2.8 \pm 11$ & $3.7 \pm 11$ & $4.4 \pm 10$ & $3.4 \pm 13$ & $3.1 \pm 12$ & $0.9 \pm 22$ & $0.4 \pm 38$ & $0.4 \pm 21$ & $0.3 \pm 30$ & $0.2 \pm 36$ & 1.9 & 82 & $3 \times 10^{-2}$ \\
\hline
\end{tabular}

See Fig. 5. Location 040-06 is in city of Tracy.

${ }^{8} \mathrm{CG}=6.0 \times 10^{-16} \mu \mathrm{Ci} / \mathrm{ml}$. 
Table 8. Plutonium and uranium on air filters - LLNL perimeter.

\begin{tabular}{|c|c|c|c|c|c|}
\hline Month & Location" & $\frac{10^{-17} \mu \mathrm{Ci} / \mathrm{ml} \pm 2 \sigma(\%)}{{ }^{239} \mathrm{Pu}^{\mathrm{b}}}$ & $\frac{10^{-7} \mu \mathrm{g} / \mathrm{m}^{3} \pm 2 \sigma(\%)}{{ }^{233} U}$ & $\frac{10^{-5} \mu \mathrm{g} / \mathrm{m}^{3} \pm 2 \sigma(\%)}{{ }^{236} \mathrm{U}}$ & $\frac{10^{-3}}{{ }^{235} \mathrm{U} / /^{230} \mathrm{U}}$ \\
\hline \multirow[t]{6}{*}{ Jan. } & 01 & $0.8 \pm 16$ & $2.1 \pm 1$ & $3.8 \pm 1$ & 5.6 \\
\hline & 02 & $1.0 \pm 19$ & $1.8 \pm 1$ & $2.7 \pm 1$ & 6.7 \\
\hline & 12 & $0.6 \pm 28$ & $3.2 \pm 2$ & $5.7 \pm 1$ & 5.6 \\
\hline & 13 & $0.9 \pm 16$ & $1.9 \pm 2$ & $3.5 \pm 1$ & 5.5 \\
\hline & 14 & $1.2 \pm 17$ & $24 \pm 1$ & $4.2 \pm 1$ & 5.7 \\
\hline & 15 & $0,8 \pm 17$ & $2.0 \pm 2$ & $3.4 \pm 1$ & 6.0 \\
\hline \multirow[t]{6}{*}{ Feb. } & 01 & $1.1 \pm 23$ & $2.3 \pm 4$ & $3.7 \pm 1$ & 6.3 \\
\hline & 02 & $1.5 \pm 15$ & $2.5 \pm 1$ & $3.8 \pm 1$ & 6.6 \\
\hline & 12 & $1.5 \pm 19$ & $3.7 \pm 2$ & $6.2 \pm 1$ & 5.9 \\
\hline & 13 & $1.3 \pm 15$ & $1.6 \pm 1$ & $2.5 \pm 1$ & 6.6 \\
\hline & 14 & $1.6 \pm 16$ & $22 \pm 1$ & $3.3 \pm 1$ & 6.6 \\
\hline & 15 & $1.8 \pm 14$ & $2.6 \pm 1$ & $3.8 \pm 1$ & 6.8 \\
\hline \multirow[t]{6}{*}{ Mar. } & 01 & $2.3 \pm 12$ & $1.6 \pm 1$ & $2.4 \pm 1$ & 6.9 \\
\hline & 02 & $2.3 \pm 11$ & $1.7 \pm 2$ & $2.5 \pm 1$ & 7.1 \\
\hline & 12 & $2.2 \pm 11$ & $2.9 \pm 2$ & $4.1 \pm 1$ & 7.1 \\
\hline & 13 & $1.7 \pm 9$ & $1.3 \pm 1$ & $1.8 \pm 1$ & 7.2 \\
\hline & 14 & $2.3 \pm 9$ & $1.8 \pm 1$ & $2.4 \pm 1$ & 7.6 \\
\hline & 15 & $1.8 \pm 9$ & $2.1 \pm 1$ & $2.9 \pm 1$ & 7.1 \\
\hline \multirow[t]{6}{*}{ Apr. } & 01 & $3.4 \pm 13$ & $2.6 \pm 1$ & $3.9 \pm 1$ & 6.8 \\
\hline & 02 & $3.5 \pm 11$ & $2.3 \pm 1$ & $3.4 \pm 1$ & 7.1 \\
\hline & 12 & $3.1 \pm 13$ & $3.8 \pm 1$ & $5.5 \pm 1$ & 6.9 \\
\hline & 13 & $2.5 \pm 11$ & $1.8 \pm 1$ & $2.6 \pm 1$ & 7.2 \\
\hline & 14 & $3.9 \pm 13$ & $3.4 \pm 1$ & $4.9 \pm 1$ & 6.9 \\
\hline & 15 & $2.9 \pm 14$ & $3.7 \pm 1$ & $5.2 \pm 1$ & 7.2 \\
\hline \multirow[t]{6}{*}{ May } & 01 & $3.0 \pm 15$ & $3.9 \pm 1$ & $5.6 \pm 1$ & 7.2 \\
\hline & 02 & $3.8 \pm 11$ & $3.6 \pm 1$ & $5.1 \pm 1$ & 7.1 \\
\hline & 12 & $2.5 \pm 13$ & $4.7 \pm 1$ & $7.1 \pm 1$ & 6.7 \\
\hline & 13 & $2.9 \pm 9$ & $4.2 \pm 1$ & $6.1 \pm 1$ & 7.0 \\
\hline & 14 & $3.2 \pm 9$ & $3.4 \pm 1$ & $4.8 \pm 1$ & 7.3 \\
\hline & 15 & $3.1 \pm 8$ & $6.4 \pm 1$ & $8.9 \pm 1$ & 7.3 \\
\hline \multirow[t]{6}{*}{ June } & 01 & $3.1 \pm 8$ & $6.4 \pm 3$ & $8.9 \pm 2$ & 7.2 \\
\hline & 02 & $4.0 \pm 7$ & $5.3 \pm 3$ & $7.4 \pm 2$ & 7.4 \\
\hline & 12 & $3.9 \pm 9$ & $11.5 \pm 3$ & $16.4 \pm 2$ & 7.1 \\
\hline & 13 & $2.6 \pm 8$ & $3.4 \pm 2$ & $4.7 \pm 2$ & 7.4 \\
\hline & 14 & $3.4 \pm 9$ & $6.4 \pm 2$ & $9.0 \pm 1$ & 7.2 \\
\hline & 15 & $2.8 \pm 8$ & $6.8 \pm 2$ & $9.5 \pm 2$ & 7.3 \\
\hline \multirow[t]{6}{*}{ July } & 01 & $2.3 \pm 9$ & $8.5 \pm 2$ & $11.6 \pm 1$ & 7.4 \\
\hline & 02 & $2.6 \pm 12$ & $6.6 \pm 3$ & $8.8 \pm 1$ & 7.5 \\
\hline & 12 & $2.6 \pm 10$ & $10.0 \pm 2$ & $14.0 \pm 1$ & 7.2 \\
\hline & 13 & lost & $3.9 \pm 3$ & $5.4 \pm 2$ & 7.3 \\
\hline & 14 & $2.8 \pm 8$ & $6.8 \pm 3$ & $9.4 \pm 2$ & 7.2 \\
\hline & 15 & $2.3 \pm 9$ & $11.5 \pm 2$ & $16.1 \pm 1$ & 7.2 \\
\hline
\end{tabular}


Table 8. (Continued).

\begin{tabular}{|c|c|c|c|c|c|}
\hline Month & Location" & $\frac{10^{-17} \mu \mathrm{Ci} / \mathrm{ml} \pm 2 \sigma(\%)}{{ }^{239} \mathrm{Pu}^{b}}$ & $\frac{10^{-7} \mu \mathrm{g} / \mathrm{m}^{3} \pm 2 \sigma(\%)}{{ }^{23} \mathrm{U}}$ & $\frac{10^{-5} \mu g / m^{3} \pm 2 \sigma(\%)}{{ }^{250} U}$ & $\frac{10^{-3}}{{ }^{235} \mathrm{U} /{ }^{230} \mathrm{U}}$ \\
\hline \multirow[t]{6}{*}{ Aug. } & 01 & $1.1 \pm 1 n$ & $7.9 \pm 1$ & $10.7 \pm 1$ & 7.1 \\
\hline & 02 & $0.9 \pm 17$ & $5.1 \pm 1$ & $7.1 \pm 1$ & 7.2 \\
\hline & 12 & $0.8 \pm 19$ & $9.0 \pm 1$ & $13.2 \pm 1$ & 7.0 \\
\hline & 13 & $0.8 \pm 13$ & $3.3 \pm 1$ & $4.6 \pm 1$ & 7.3 \\
\hline & 14 & $1.4 \pm 10$ & $7.0 \pm 1$ & $9.7 \pm 1$ & 7.2 \\
\hline & 15 & $0.7 \pm 15$ & $9.7 \pm 1$ & $13.4 \pm 1$ & 7.3 \\
\hline \multirow[t]{6}{*}{ Sept. } & 01 & $0.8 \pm 16$ & $5.9 \pm 2$ & $8,3 \pm 1$ & 7.2 \\
\hline & 02 & $0.8 \pm 16$ & $5.6 \pm 1$ & $7.9 \pm 1$ & 7.2 \\
\hline & 12 & $1.2 \pm 15$ & $8.0 \pm 2$ & $11.6 \pm 1$ & 7.0 \\
\hline & 13 & $0.6 \pm 17$ & $4.0 \pm 2$ & $5.7 \pm 1$ & 7.2 \\
\hline & 14 & $1.0 \pm 18$ & $6.2 \pm 2$ & $8.7 \pm 1$ & 7.2 \\
\hline & 15 & $0.6 \pm 14$ & $7.2 \pm 1$ & $10.1 \pm 1$ & 7.3 \\
\hline \multirow[t]{6}{*}{ Oct. } & 01 & $0.6 \pm 22$ & $8.7 \pm 2$ & $13.4 \pm 1$ & 6.6 \\
\hline & 02 & $0.4 \pm 42$ & $6.0 \pm 1$ & $8.5 \pm 1$ & 7.1 \\
\hline & 12 & $0.3 \pm 34$ & $8.0 \pm 1$ & $11.6 \pm 1$ & 7.0 \\
\hline & 13 & $0.4 \pm 27$ & $3.7 \pm 2$ & $5.2 \pm 1$ & 7.2 \\
\hline & 14 & $0.6 \pm 35$ & $6.3 \pm 2$ & $8.9 \pm 1$ & 7.2 \\
\hline & 15 & $0.3 \pm 33$ & $5.4 \pm 1$ & $7.7 \pm 1$ & 7.2 \\
\hline \multirow[t]{6}{*}{ Nov. } & 01 & $0.3 \pm 52$ & $2.6 \pm 2$ & $3.7 \pm 1$ & 6.9 \\
\hline & 02 & $0.2 \pm 34$ & $3.1 \pm 2$ & $4.6 \pm 1$ & 6.9 \\
\hline & 12 & $0.3 \pm 30$ & $7.8 \pm 2$ & $11.2 \pm 1$ & 7.1 \\
\hline & 13 & $0.2 \pm 26$ & $1.9 \pm 1$ & $2.8 \pm 1$ & 7.0 \\
\hline & 14 & $0.2 \pm 31$ & $2.5 \pm 1$ & $3.7 \pm 1$ & 6.8 \\
\hline & 15 & $0.2 \pm 23$ & $2.6 \pm 2$ & $3.7 \pm 1$ & 7.2 \\
\hline \multirow[t]{6}{*}{ Dec. } & 01 & $0.1 \pm 49$ & $15.8 \pm 1$ & $67.7 \pm 2$ & 2.4 \\
\hline & 02 & $0.1 \pm 43$ & $9.1 \pm 1$ & $33.2 \pm 2$ & 2.8 \\
\hline & 12 & $0.2 \pm 39$ & $11.9 \pm 1$ & $35.8 \pm 1$ & 3.4 \\
\hline & 13 & $0.2 \pm 31$ & $5.7 \pm 1$ & $18.5 \pm 2$ & 3.1 \\
\hline & 14 & $0.2 \pm 37$ & $15.6 \pm 2$ & $69.7 \pm 2$ & 2.3 \\
\hline & 15 & $0.2 \pm 27$ & $5.4 \pm 2$ & $19.8 \pm 1$ & 2.8 \\
\hline
\end{tabular}

Annual averages:

\begin{tabular}{|c|c|c|c|c|c|c|c|c|c|}
\hline \multirow[b]{2}{*}{ Location } & \multicolumn{3}{|c|}{$10^{-17} \mu \mathrm{Ci} / \mathrm{ml}$} & \multicolumn{3}{|c|}{$10^{-7} \mu \mathrm{g} / \mathrm{m}^{3}$} & \multicolumn{3}{|c|}{$10^{-5} \mu \mathrm{g} / \mathrm{m}^{3}$} \\
\hline & ${ }^{2^{39}} \mathbf{P u}$ & $\%$ SDM & $\% \mathrm{CG}^{\mathrm{C}}$ & $\overline{{ }^{25} \mathbf{U}}$ & $\%$ SDM & $\% \subset G^{d}$ & $\overline{{ }^{236} \mathrm{U}}$ & $\%$ SDM & $\% \mathrm{CG}^{\mathrm{t}}$ \\
\hline 01 & 1.6 & 74 & $3 \times 10^{-2}$ & 5.7 & 73 & $3 \times 10^{-5}$ & 12.0 & 150 & $8 \times 10^{-4}$ \\
\hline 02 & 1.8 & 81 & $3 \times 10^{-2}$ & 4.4 & 52 & $2 \times 10^{-5}$ & 7.9 & 105 & $5 \times 10^{-4}$ \\
\hline 12 & 1.6 & 78 & $3 \times 10^{-2}$ & 7.0 & 46 & $4 \times 10^{-5}$ & 11.9 & 72 & $8 \times 10^{-4}$ \\
\hline 13 & 1.3 & 78 & $2 \times 10^{-2}$ & 3.0 & 44 & $2 \times 10^{-5}$ & 5.3 & 83 & $4 \times 10^{-4}$ \\
\hline 14 & 1.8 & 70 & $3 \times 10^{-2}$ & 5.3 & 71 & $3 \times 10^{-9}$ & 11.6 & 160 & $8 \times 10^{-4}$ \\
\hline 15 & 1.5 & 77 & $2 \times 10^{-2}$ & 5.4 & 56 & $3 \times 10^{-5}$ & 8.7 & 63 & $6 \times 10^{-4}$ \\
\hline
\end{tabular}

'See Fig. 3.

bIn all tables activity listed as ${ }^{209} \mathrm{Pu}$ includes activity due to ${ }^{200} \mathrm{Pu}$ isotope.

${ }^{\circ} \mathrm{CG}=6.0 \times 10^{-14} \mu \mathrm{Cl} / \mathrm{ml}$ for ${ }^{239} \mathrm{Pu}$ (soluble) activity in air.

${ }^{\mathrm{d}} \mathrm{CG}=1.9 \mu \mathrm{g} / \mathrm{m}^{3}$ for ${ }^{23} \mathrm{U}$ (insoluble) activity in air.

'CG $=15 \mu g / \mathrm{m}^{3}$ for ${ }^{200} \mathrm{U}$ (insoluble) activity in air. 
Table 9. Plutonium, cesium, and uranium on air filters-Site 300.

\begin{tabular}{|c|c|c|c|c|c|c|}
\hline Month & $\begin{array}{c}10^{-17} \mu \mathrm{Ci} / \mathrm{ml} \\
\pm 2 \sigma(\%) \\
{ }^{239} \mathrm{Pu}\end{array}$ & $\begin{array}{c}10^{-15} \mu \mathrm{Ci} / \mathrm{ml} \\
\pm 2 \sigma(\%) \\
{ }^{137} \mathrm{Cs}\end{array}$ & $\frac{10^{-2}}{{ }^{239} \mathrm{Pu} /{ }^{137} \mathrm{Cs}}$ & $\begin{array}{c}10^{-7} \mu \mathrm{g} / \mathrm{m}^{3} \\
\pm 2 \sigma(\%) \\
{ }^{235} \mathrm{U}\end{array}$ & $\begin{array}{r}10^{-5} \mu \mathrm{g} / \mathrm{m}^{3} \\
+\quad 2 \sigma(\%) \\
{ }^{238} \mathrm{U}\end{array}$ & $\frac{10^{-3}}{{ }^{235} U /^{238} U}$ \\
\hline Jan. & $1.4 \pm 5$ & $0.9 \pm 7$ & 1.6 & $8.2 \pm 3$ & $36.0 \pm 2$ & 2.3 \\
\hline Feb. & $2.5 \pm 6$ & $1.3 \pm 5$ & 1.9 & $156 \pm 3$ & $681 \pm 2$ & 2.3 \\
\hline Mar. & $2.4 \pm 6$ & $1.8 \pm 3$ & 1.3 & $3.2 \pm 2$ & $12.1 \pm 2$ & 2.7 \\
\hline Apr. & $3.5 \pm 8$ & $3.4 \pm 5$ & 1.0 & $5.0 \pm 1$ & $17.0 \pm 2$ & 3.0 \\
\hline May & $3.3 \pm 6$ & $3.5 \pm 4$ & 0.1 & $4.6 \pm 1$ & $8.7 \pm 1$ & 5.3 \\
\hline June & $4.5 \pm 3$ & $3.8 \pm 3$ & 1.2 & $5.0 \pm 1$ & $12.3 \pm 2$ & 4.1 \\
\hline July & $1.9 \pm 15$ & $3.1 \pm 3$ & 0.4 & $4.1 \pm 2$ & $7.7 \pm 2$ & 5.4 \\
\hline Aug. & $1.3 \pm 6$ & $1.1 \pm 3$ & 1.2 & $3.4 \pm 1$ & $5.7 \pm 1$ & 6.0 \\
\hline Sept. & $0.8 \pm 7$ & $0.6 \pm 5$ & 1.3 & $4.3 \pm 1$ & $8.5 \pm 2$ & 5.1 \\
\hline Oct. & $0.4 \pm 11$ & $0.3 \pm 6$ & 1.3 & $4.5 \pm 1$ & $8.1 \pm 1$ & 5.6 \\
\hline Nov. & $0.4 \pm 7$ & $0.3 \pm 11$ & 1.3 & $4.1 \pm 1$ & $15.2 \pm 2$ & 2.7 \\
\hline Dec. & $0.5 \pm 7$ & $0.2 \pm 16$ & 2.5 & $26.1 \pm 2$ & $128 \pm 2$ & 2.1 \\
\hline Annual average & 1.9 & 1.7 & & 19 & 78.4 & \\
\hline$\%$ SDM & 71 & 82 & & 229 & 246 & \\
\hline CG & $6.0 \times 10^{-14} \mu \mathrm{Ci} / \mathrm{ml}$ & $5.0 \times 10^{-10} \mu \mathrm{Ci} / \mathrm{ml}$ & & $1.9 \mu \mathrm{g} / \mathrm{m}^{3}$ & $15 \mu g / m^{3}$ & \\
\hline$\% \mathrm{CG}$ & $3 \times 10^{-2}$ & $3 \times 10^{4}$ & & $1 \times 10^{-4}$ & $5 \times 10^{-3}$ & \\
\hline
\end{tabular}


Table 10. Tritium (HTO) in air - LLNL and Livermore Valley.

\begin{tabular}{|c|c|c|c|c|c|c|c|c|c|c|c|c|c|c|c|c|}
\hline \multirow[b]{2}{*}{ Location } & \multicolumn{13}{|c|}{$10^{-11} \mu \mathrm{Ci} / \mathrm{ml}$} & \multirow[b]{2}{*}{$\%$ SDM } & \multirow[b]{2}{*}{$\% \mathbf{C G}^{b}$} & \multirow{2}{*}{$\begin{array}{l}\text { Calculated' adult } \\
\text { whole-body dose, } \\
\text { mrem }\end{array}$} \\
\hline & Jan. & Feb. & Mar. & Apr. & May & June & July & Aug. & Sept. & Oct. & Nov. & Dec. & $\overline{\text { Average }}$ & & & \\
\hline \multicolumn{17}{|l|}{ Perimeter } \\
\hline 01 & 8.3 & 1.8 & 26 & 21 & 1.3 & 1.5 & 0.8 & 1.0 & 0.9 & 2.7 & 2.4 & 20.4 & 3.8 & 146 & $2 \times 10^{-2}$ & $4.9 \times 10^{-2}$ \\
\hline 02 & 9.6 & 5.9 & 4.0 & 3.1 & 1.2 & 1.6 & 0.8 & 1.3 & 1.7 & 2.6 & 2.8 & $2.7^{4}$ & 3.1 & 80 & $2 \times 10^{-2}$ & $4.0 \times 10^{-2}$ \\
\hline 12 & 8.1 & 13.3 & 4.0 & 4.2 & 1.8 & 3.5 & lost & lost & 2.8 & 3.3 & 5.4 & 28.7 & 7.5 & 109 & $4 \times 10^{-2}$ & $9.7 \times 10^{-2}$ \\
\hline 13 & 4.0 & 22 & 1.6 & 1.6 & 0.7 & 0.9 & 0.2 & 0.1 & 0.7 & 1.8 & 3.8 & 22.8 & 3.4 & 186 & $2 \times 10^{-2}$ & $4.4 \times 10^{-2}$ \\
\hline 14 & 4.6 & 3.1 & 3.4 & 29 & 2.6 & 2.0 & 1.7 & 20 & 2.1 & 2.1 & 2.4 & 21.9 & 4.2 & 133 & $2 \times 10^{-2}$ & $5.4 \times 10^{-2}$ \\
\hline 15 & 4.3 & 3.1 & 1.9 & 2.3 & 1.6 & 1.4 & 1.1 & 0.9 & 1.6 & 1.7 & 2.6 & 38.7 & 5.1 & 208 & $3 \times 10^{-2}$ & $6.6 \times 10^{-2}$ \\
\hline \multicolumn{17}{|l|}{ Valley } \\
\hline S1 & 4.8 & 1.2 & 1.5 & 2.1 & 0.9 & 0.7 & 0.5 & 0.3 & 0.9 & $\operatorname{los} t$ & 20 & $0.8^{d}$ & 1.4 & 88 & $1 \times 10^{-2}$ & $1.8 \times 10^{-2}$ \\
\hline S2 & 4.2 & 0.4 & 0.7 & 1.4 & 1.7 & 1.6 & 0.9 & 0.05 & 0.7 & 0.5 & 1.6 & 15.7 & 2.4 & 176 & $1 \times 10^{-2}$ & $3.1 \times 10^{-2}$ \\
\hline
\end{tabular}

See Figs. 3 and 5.

${ }^{b} \mathrm{CG}=20 \times 10^{-7} \mu \mathrm{Cl} / \mathrm{ml}$.

'Doses are calculated using methods in U.S. NRC Regulatory Guide 1.109 unless otherwise stated.

${ }^{\mathrm{d}}$ Locations 02 and $\mathrm{S1}$ were inoperative during last two weeks of December. 
Table 11. Various radionuclides in soil - Livermore Valley (sampling depth $=0-5 \mathrm{~cm}$ ).

\begin{tabular}{|c|c|c|c|c|c|}
\hline \multirow[b]{2}{*}{ Location" } & \multicolumn{3}{|c|}{$\mu \mathrm{Ci} / \mathrm{dry} \mathrm{g} \pm 2 \sigma(\%)$} & \multicolumn{2}{|c|}{$\mu_{B} / \mathrm{dry} B \pm 2 \sigma(\%)$} \\
\hline & ${ }^{29} \mathrm{Pu}\left(10^{-8}\right)$ & ${ }^{40} \mathrm{~K}\left(10^{-5}\right)$ & ${ }^{137} \operatorname{Cs}\left(10^{-7}\right)$ & ${ }^{22} \mathrm{Th}$ & ${ }^{238} \mathbf{U}$ \\
\hline 852 & $5.2 \pm 9$ & $0.9 \pm 8$ & $2.9 \pm 6$ & $4.9 \pm 14$ & $2.9 \pm 21$ \\
\hline 853 & $5.3 \pm 9$ & $0.9 \pm 5$ & $3.7 \pm 4$ & $4.6 \pm 7$ & $2.7 \pm 41$ \\
\hline 854 & $4.4 \pm 9$ & $1.2 \pm 8$ & $2.9 \pm 10$ & $5.8 \pm 12$ & $3.0 \pm 91$ \\
\hline 855 & $0.7 \pm 20$ & $1.3 \pm 8$ & $0.6 \pm 33$ & $6.6 \pm 13$ & $4.2 \pm 41$ \\
\hline 856 & $3.4 \pm 9$ & $0.9 \pm 9$ & $2.3 \pm 10$ & $4.0 \pm 18$ & $2.5 \pm 62$ \\
\hline 857 & $0.3 \pm 46$ & $0.9 \pm 3$ & $0.2 \pm 35$ & $6.1 \pm 4$ & $3.9 \pm 17$ \\
\hline 858 & $3.1 \pm 10$ & $0.5 \pm 6$ & $2.1 \pm 10$ & $3.7 \pm 7$ & $2.7 \pm 20$ \\
\hline 859 & $0.7 \pm 19$ & $1.0 \pm 4$ & $0.5 \pm 17$ & $4.6 \pm 6$ & $3.6 \pm 16$ \\
\hline 866 & $1.6 \pm 11$ & $1.2 \pm 8$ & $0.9 \pm 31$ & $5.7 \pm 15$ & $4.2 \pm 38$ \\
\hline 867 & $3.6 \pm 10$ & $1.1 \pm 8$ & $1.6 \pm 14$ & $5.7 \pm 11$ & $2.1 \pm 72$ \\
\hline 868 & $1.6 \pm 13$ & $1.5 \pm 6$ & $1.0 \pm 18$ & $6.9 \pm 9$ & $5.1 \pm 21$ \\
\hline 869 & $11.3 \pm 7$ & $1.2 \pm 6$ & $3.5 \pm 9$ & $6.0 \pm 10$ & $2.1 \pm 72$ \\
\hline 870 & $5.8 \pm 8$ & $1.4 \pm 7$ & $2.8 \pm 19$ & $6.5 \pm 10$ & $1.8 \pm 62$ \\
\hline 871 & $5,0 \pm 10$ & $1.1 \pm 6$ & $2.4 \pm 11$ & $5.6 \pm 8$ & $2.7 \pm 74$ \\
\hline 872 & $6.0 \pm 8$ & $1.5 \pm 3$ & $26 \pm 5$ & $4.3 \pm 7$ & $3.0 \pm 33$ \\
\hline 873 & $1.6 \pm 14$ & $1.4 \pm 5$ & $0.9 \pm 10$ & $6.7 \pm 5$ & $4.2 \pm 20$ \\
\hline 874 & $3.9 \pm 10$ & $1.0 \pm 6$ & $1.0 \pm 19$ & $5.9 \pm 12$ & $2.9 \pm 57$ \\
\hline 875 & $1.6 \pm 13$ & $1.4 \pm 3$ & $0.9 \pm 10$ & $5.7 \pm 5$ & $4.2 \pm 8$ \\
\hline 876 & $17,9 \pm 7$ & $0.9 \pm 6$ & $1.3 \pm 17$ & $5.9 \pm 10$ & $3.6 \pm 33$ \\
\hline 877 & $4.0 \pm 11$ & $1.0 \pm 10$ & $2.1 \pm 12$ & $5.1 \pm 15$ & $3.5 \pm 33$ \\
\hline 878 & $1.2 \pm 13$ & $0.6 \pm 6$ & $0.4 \pm 21$ & $3.8 \pm 9$ & $2.4 \pm 72$ \\
\hline 879 & $7.1 \pm 6$ & $1.6 \pm 6$ & $3.9 \pm 4$ & $6.5 \pm 8$ & $4.7 \pm 21$ \\
\hline
\end{tabular}

"See Fig. 6.

Table 12. Plutonium, cesium and uranium in soil - Site 300 (sampling depth $=0-5 \mathrm{~cm}$ ).

\begin{tabular}{|c|c|c|c|}
\hline \multirow[b]{2}{*}{ Location" } & \multicolumn{2}{|c|}{$\mu \mathrm{Cl} / \mathrm{dry} \mathrm{B} \pm 2 \sigma(\%)$} & \multirow{2}{*}{$\frac{\mu \mathrm{g} / \mathrm{dry} \mathrm{g} \pm 2 \sigma(\%)}{{ }^{238} \mathrm{U}}$} \\
\hline & ${ }^{209} \mathrm{Pu}\left(10^{-9}\right)$ & ${ }^{137} \mathrm{Cs}\left(10^{-7}\right)$ & \\
\hline 840 & $3.1 \pm 11$ & $1.7 \pm 8$ & $46.8 \pm 11$ \\
\hline 841 & $2.0 \pm 13$ & $1.2 \pm 14$ & $46.2 \pm 8$ \\
\hline 842 & $6.7 \pm 10$ & $3.3 \pm 7$ & $3.9 \pm 25$ \\
\hline 845 & $0.4 \pm 29$ & $0.3 \pm 32$ & $7.4 \pm 15$ \\
\hline 844 & $4.2 \pm 9$ & $2.2 \pm 12$ & $5.1 \pm 24$ \\
\hline 845 & $3.5 \pm 9$ & $2.0 \pm 9$ & $5.1 \pm 24$ \\
\hline 846 & $4.3 \pm 8$ & $2.4 \pm 6$ & $5.4 \pm 12$ \\
\hline 847 & $3.6 \pm 9$ & $2.0 \pm 9$ & $6.3 \pm 13$ \\
\hline 848 & $6.6 \pm 8$ & $3.1 \pm 5$ & $5.7 \pm 9$ \\
\hline 849 & $3.3 \pm 12$ & $4.4 \pm 7$ & $272 \pm 6$ \\
\hline 850 & $5.7 \pm 9$ & $3.6 \pm 5$ & $4.5 \pm 58$ \\
\hline 865 & $5.4 \pm 8$ & $2.7 \pm 11$ & $5.4 \pm 21$ \\
\hline
\end{tabular}

See Fig. 7. 
Table 13. Various radionuclides in effluents - LLNL and Livermore Water Reclamation Plant.

\begin{tabular}{|c|c|c|c|c|c|c|}
\hline \multirow[b]{2}{*}{ Month } & \multicolumn{2}{|c|}{$\begin{array}{c}10^{-6} \mu \mathrm{Ci} / \mathrm{ml} \pm 2 \sigma(\%) \\
\text { HTO }\end{array}$} & \multicolumn{2}{|c|}{$\begin{array}{c}10^{-11} \mu \mathrm{Ci} / \mathrm{ml} \pm 2 \sigma(\%) \\
{ }^{137} \mathrm{C}_{s}\end{array}$} & \multicolumn{2}{|c|}{ 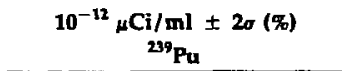 } \\
\hline & LLNL & LWRP & LLNL & LWRP & LLNL & LWRP \\
\hline Jan. & $13.2 \pm 43$ & $9.8 \pm 72$ & $23.6 \pm 7$ & $6.4 \pm 19$ & \pm 5 & $10.9 \pm 14$ \\
\hline Feb. & $18.3 \pm 44$ & $12.1 \pm 74$ & $23.0 \pm 7$ & $7.8 \pm 13$ & \pm 4 & $11.9 \pm 14$ \\
\hline Mar. & $3.0 \pm 54$ & $0.6 \pm 77$ & $197 \pm 3$ & $13.5 \pm 11$ & \pm 5 & $24.3 \pm 10$ \\
\hline Apr. & $2.8 \pm 71$ & $0.7 \pm 84$ & $17.9 \pm 14$ & $2.5 \pm 100$ & \pm 6 & $8.2 \pm 14$ \\
\hline May & $3.8 \pm 68$ & $0.7 \pm 80$ & $4.2 \pm 36$ & $1.9 \pm 75$ & \pm 7 & $2.9 \pm 28$ \\
\hline June & $1.9 \pm 43$ & $0.7 \pm 91$ & $4.3 \pm 58$ & $2.1 \pm 98$ & \pm 10 & $2.7 \pm 30$ \\
\hline July & $3.5 \pm 36$ & $0.6 \pm 87$ & $4.5 \pm 33$ & $21 \pm 14$ & $5.5 \pm 21$ & $1.5 \pm 38$ \\
\hline Aug. & $41.0 \pm 21$ & $5.3 \pm 37$ & $1.9 \pm 44$ & $2.3 \pm 49$ & $4.2 \pm 19$ & $1.0 \pm 46$ \\
\hline Sept. & $18.3 \pm 15$ & $1.8 \pm 46$ & $2.5 \pm 31$ & $0.7 \pm 100$ & $5.1 \pm 19$ & $1.0 \pm 34$ \\
\hline Oct. & $4.2 \pm 37$ & $0.5 \pm 82$ & $3.0 \pm 26$ & $2.2 \pm 51$ & $23.6 \pm 16$ & $0.5 \pm 82$ \\
\hline Nov. & $6.8 \pm 29$ & $0.7 \pm 59$ & $6.2 \pm 50$ & $0.6 \pm 100$ & $37.6 \pm 11$ & $2.6 \pm 41$ \\
\hline Dec. & $6.3 \pm 31$ & $0.7 \pm 71$ & $5.8 \pm 29$ & $10 \pm 50$ & $21.5 \pm 11$ & $3.0 \pm 29$ \\
\hline Annual average & 10.3 & 2.8 & 24,5 & 5.9 & 153 & 5.9 \\
\hline$\%$ SDM & 110 & 142 & 224 & 106 & 151 & 119 \\
\hline $\mathrm{CG}(\mu \mathrm{Ci} / \mathrm{ml})$ & 0.1 & $3 \times 10^{-3}$ & $4.0 \times 10^{-1}$ & $2.0 \times 10^{-5}$ & $1.0 \times 10^{-4}$ & $5.0 \times 10^{-6}$ \\
\hline$\%$ CG & $1.0 \times 10^{-2}$ & $9 \times 10^{-2}$ & $6 \times 10^{-5}$ & $3.0 \times 10^{-4}$ & $2 \times 10^{-4}$ & $1 \times 10^{-4}$ \\
\hline
\end{tabular}

Table 14. Gross alpha activity in water - Livermore Valley.

\begin{tabular}{|c|c|c|c|c|c|c|}
\hline \multirow[b]{2}{*}{ Location" } & \multirow{2}{*}{$\begin{array}{c}\text { Number of } \\
\text { samples }\end{array}$} & \multicolumn{3}{|c|}{$10^{-9} \mu \mathrm{Ci} / \mathrm{ml} \pm 2 \sigma(\%)$} & \multirow[b]{2}{*}{$x_{S D M}$} & \multirow[b]{2}{*}{${ }_{*} \mathrm{CG}^{\mathrm{b}}$} \\
\hline & & Maximum & Minimum & Average & & \\
\hline 11 & 4 & $3.4 \pm 2.5$ & $0.8 \pm 4.0$ & $2.7 \pm 3.4$ & 78 & 0.9 \\
\hline 15 & 4 & $\leq 5.3$ & $-0.2 \pm 2.4^{\mathrm{C}}$ & $\leq 1.8 \pm 1.7$ & 143 & $\leq 6$ \\
\hline 16 & 4 & $7.9 \pm 10.2$ & $0.0 \pm 2.3$ & $\leq 4.9 \pm 9.7$ & 70 & $\leq 16$ \\
\hline 19 & 4 & $\leq 5.3$ & $0.6 \pm 4.2$ & $\leq 2.1 \pm 3.3$ & 105 & $\leq 7$ \\
\hline 20 & 6 & $3.3 \pm 2.0$ & $-0.1 \pm 0.8$ & $\leq 2.2 \pm 2.0$ & 86 & $\leq 7$ \\
\hline 24 & 4 & $\leq 5.3$ & $1.3 \pm 2.0$ & $\leq 2.8 \pm 2.5$ & 67 & $\leq 9$ \\
\hline 26 & 4 & $\leq 5.3$ & $-0.3 \pm 3.3$ & $\leq 1.6 \pm 3.7$ & 160 & $\leq 5$ \\
\hline 29 & 4 & $\leq 5.3$ & $0.0 \pm 1.3$ & $\leq 1.5 \pm 2.2$ & 169 & $\leq 5$ \\
\hline 31 & 4 & $6.3 \pm 6.2$ & $0.0 \pm 2.4$ & $2.6 \pm 4.4$ & 114 & 0.9 \\
\hline 32 & 4 & $\leq 3.6$ & $-0.6 \pm 3.5$ & $\leq 1.1 \pm 2.5$ & 170 & $\leq 4$ \\
\hline 33 & 4 & $\leq 5.3$ & $-1.3 \pm 4.0$ & $\leq 1.3 \pm 3.9$ & 206 & $\leq 4$ \\
\hline 34 & 3 & $\leq 5.3$ & $-2.6 \pm 5.8$ & $\leq 1.8 \pm 5.4$ & 224 & $\leq 6$ \\
\hline
\end{tabular}

"See Fig. 9.

${ }^{b} \mathrm{CG}=3.0 \times 10^{-6} \mu \mathrm{Ci} / \mathrm{ml}$.

'Negative values indicate the reported number is below delection limit. 
Table 15. Gross alpha activity in water - Site 300 .

\begin{tabular}{|c|c|c|c|c|c|c|}
\hline \multirow[b]{2}{*}{ Location" } & \multirow{2}{*}{$\begin{array}{c}\text { Number of } \\
\text { samples }\end{array}$} & \multicolumn{3}{|c|}{$10^{-9} \mu \mathrm{Ci} / \mathrm{ml} \pm 2 \sigma(\%)$} & \multirow[b]{2}{*}{$\%$ SDM } & \multirow[b]{2}{*}{$\% \mathbf{C G}^{\mathrm{b}}$} \\
\hline & & Maximum & Minimum & Average & & \\
\hline 01 & 4 & $4.4 \pm 4.5$ & $1.2 \pm 4.2$ & $\leq 3.2 \pm 3.7$ & 49 & $\leq 11$ \\
\hline 02 & 4 & $6 \pm 4.4$ & $3.7 \pm 1.8$ & $\leq 4.6 \pm 4.2$ & 22 & $\leq 18$ \\
\hline 03 & 4 & $\leq 4.4$ & $-2.5 \pm 5.2^{\mathrm{c}}$ & $\leq 1.4 \pm 38$ & 46 & 5 \\
\hline 04 & 4 & $51.1 \pm 6.8$ & $\leq 4.4$ & $\leq 33.8 \pm 5.6$ & 65 & $\leq 0.2^{d}$ \\
\hline 05 & 4 & $\leq 4.4$ & $0.8 \pm 6$ & $\leq 1.8 \pm 3.9$ & 92 & $\leq 6$ \\
\hline 06 & 4 & $\leq 4.4$ & $0.4 \pm 2.6$ & $\leq 1.7 \pm 2.9$ & 110 & $\leq 6$ \\
\hline 07 & 4 & $\leq 8.8$ & $-0.4 \pm 4.6$ & $\leq 2.3 \pm 5.3$ & 192 & $\leq 8$ \\
\hline 11 & 4 & $22 \pm 19$ & $0.7 \pm 6.8$ & $\leq 7 \pm 12$ & 146 & $\leq 23$ \\
\hline 14 & 3 & $10 \pm 5.8$ & $4.9 \pm 7.2$ & $7.8 \pm 7.2$ & 34 & 26 \\
\hline 20 & 6 & $\leq 8.8$ & $0.8 \pm 1.0$ & $3.2 \pm 2.0$ & 97 & 11 \\
\hline 21 & 4 & $105 \pm 28$. & $\leq 4.4$ & $\leq 44 \pm 24$ & 107 & $\leq 0.2^{c}$ \\
\hline
\end{tabular}

'See Fig. 10.

${ }^{\mathrm{b}} \mathrm{CG}=3.0 \times 10^{-\mathrm{s}} \mu \mathrm{Ci} / \mathrm{ml}$.

'Negative values indicate the reported number is below detection limit.

${ }^{\mathrm{d}}$ Specific analyses of these samples demonstrate that all the activity is due to naturally occurring uranium for which the $\mathrm{CG}$ is $2.0 \times 10^{-5} \mu \mathrm{Ci} / \mathrm{ml}$.

Table 16. Gross beta activity in water - Livermore Valley.

\begin{tabular}{|c|c|c|c|c|c|c|}
\hline \multirow[b]{2}{*}{ Location" } & \multirow{2}{*}{$\begin{array}{c}\text { Number of } \\
\text { samples }\end{array}$} & \multicolumn{3}{|c|}{$10^{-9} \mu \mathrm{Cl} / \mathrm{ml} \pm 2 \sigma(\%)$} & \multirow[b]{2}{*}{$\%$ SDM } & \multirow[b]{2}{*}{$\% \mathbf{C G}^{b}$} \\
\hline & & Maximum & Minimum & Average & & \\
\hline 11 & 4 & $\leq 12$ & $2.3 \pm 3.0$ & $\leq 6.3 \pm 4.4$ & 69 & $\leq 2$ \\
\hline 15 & 4 & $\leq 12$ & $2.1 \pm 5.0$ & $\leq 5.1 \pm 3.6$ & 91 & $\leq 2$ \\
\hline 16 & 4 & $29.1 \pm 19.6$ & $4.6 \pm 6.0^{\mathrm{c}}$ & $\leq 14.8 \pm 11.9$ & 70 & $\leq 5$ \\
\hline 19 & 4 & $\leq 12$ & $0.7 \pm 1.0$ & $\leq 5.0 \pm 8.8$ & 105 & $\leq 2$ \\
\hline 20 & 6 & $20 \pm 6$ & $7.3 \pm 2.7$ & $\leq 12.5 \pm 11.1$ & 38 & $\leq 4$ \\
\hline 24 & 4 & $\leq 12$ & $3.9 \pm 3.0$ & $\leq 7.4 \pm 4.6$ & 55 & $\leq 2$ \\
\hline 26 & 4 & $\leq 12$ & $3.1 \pm 2.2$ & $\leq 6.0 \pm 3.3$ & 69 & $\leq 2$ \\
\hline 29 & 4 & $\leq 12$ & $2.1 \pm 2.6$ & $\leq 6.0 \pm 4.4$ & $\mathbf{7 3}$ & $\leq 2$ \\
\hline 31 & 4 & $18 \pm 6$ & $1.6 \pm 2.0$ & $6.3 \pm 4.5$ & 125 & 2 \\
\hline 32 & 4 & $42 \pm 9$ & $1.8 \pm 5.9$ & $12.3 \pm 5.6$ & 161 & 4 \\
\hline 33 & 4 & $\leq 12$ & $3.7 \pm 6.8$ & $\leq 7.1 \pm 5.3$ & 51 & $\leq 2$ \\
\hline 34 & 3 & $\leq 12$ & $8.7 \pm 2.4$ & $\leq 12.9 \pm 2.7$ & 36 & $\leq 4$ \\
\hline
\end{tabular}

'See Fig. 9.

${ }^{\mathrm{b}} \mathrm{CG}=3.0 \times 10^{-7} \mu \mathrm{Ci} / \mathrm{ml}$. 
Table 17. Gross beta activity in water-Site 300 .

\begin{tabular}{|c|c|c|c|c|c|c|}
\hline \multirow[b]{2}{*}{ Location" } & \multirow{2}{*}{$\begin{array}{c}\text { Number of } \\
\text { amples }\end{array}$} & \multicolumn{3}{|c|}{$10^{-9} \mu \mathrm{Ci} / \mathrm{ml} \pm 2 \sigma(\%)$} & \multirow[b]{2}{*}{$x_{\mathrm{SDM}}$} & \multirow[b]{2}{*}{$\% \mathrm{CG}^{\mathrm{b}}$} \\
\hline & & Maximum & Minimum & Average & & \\
\hline 01 & 4 & $\leq 10$ & $5.0 \pm 2.0$ & $\leq 7.1 \pm 46$ & 35 & $\leq 2$ \\
\hline 02 & 4 & $22 \pm 3$ & $7.9 \pm 2.5$ & $\leq 12.3$ & 53 & 4 \\
\hline $\mathbf{0 3}$ & 4 & $25 \pm 3$ & $\leq 10$ & $\leq 14.4$ & 49 & $\leq 5$ \\
\hline 04 & 4 & $34.5 \pm 15.6$ & $7 \pm 10$ & $\leq 20.6 \pm 9.7$ & 60 & 7 \\
\hline 05 & 4 & $11.6 \pm 14.2$ & $7.2 \pm 3.1$ & $\leq 9.3 \pm 9.2$ & 21 & 3 \\
\hline 06 & 4 & $16 \pm 9$ & $3.4 \pm 2.7$ & $8.1 \pm 5.2$ & 68 & 3 \\
\hline 07 & 4 & $\leq \mathbf{2 1}$ & $10.7 \pm 2.4$ & $\leq 15.8 \pm 6.7$ & 27 & 5 \\
\hline 11 & 4 & $47.8 \pm 4.2$ & $4.4 \pm 2.4$ & $\leq 17.4 \pm 4.4$ & 117 & 6 \\
\hline 14 & 3 & $48 \pm 8$ & $5.5 \pm 1.9$ & $21.6 \pm 5.1$ & 107 & 7 \\
\hline 20 & 6 & $\leq \mathbf{2 1}$ & $4.5 \pm 3.2$ & $\leq 10.5 \pm 3.6$ & 58 & $\leq 4$ \\
\hline 21 & 4 & $76.7 \pm 31.5$ & $12.4 \pm 5.0$ & $36.4 \pm 16.6$ & 84 & 12 \\
\hline
\end{tabular}

'See Fig, 10.

${ }^{b} \mathrm{CG}=3.0 \times 10^{-7} \mu \mathrm{Ci} / \mathrm{ml}$.

Table 18. Tritium (HTO) in water - Livermore Valley.

\begin{tabular}{|c|c|c|c|c|c|c|c|}
\hline \multirow[b]{2}{*}{ Location' } & \multirow{2}{*}{$\begin{array}{c}\text { Number } \\
\text { of samples }\end{array}$} & \multicolumn{3}{|c|}{$10^{-7} \mu \mathrm{Ci} / \mathrm{ml} \pm 2 \sigma(\%)$} & \multirow[b]{2}{*}{$\%$ SDM } & \multirow[b]{2}{*}{$\% \mathbf{C G}^{\mathrm{b}}$} & \multirow{2}{*}{$\begin{array}{l}\text { Calculated } \\
\text { adult whole- } \\
\text { body dose, } \\
\text { mrem }\end{array}$} \\
\hline & & Maximum & Minimum & Average & & & \\
\hline 11 & 4 & $0.8 \pm 8$ & $0.7 \pm 8$ & 0.7 & 7 & $2 \times 10^{-3}$ & $0.5 \times 10^{-2}$ \\
\hline 15 & 4 & $0.9 \pm 9$ & $0.6 \pm 14$ & 0.7 & 18 & $2 \times 10^{-3}$ & $0.5 \times 10^{-2}$ \\
\hline 16 & 4 & $21.6 \pm 3$ & $2.3 \pm 4$ & 8.2 & 112 & $27 \times 10^{-3}$ & $6.3 \times 10^{-2}$ \\
\hline 19 & 4 & $0.8 \pm 9$ & $0.4 \pm 17$ & 0.6 & 30 & $2 \times 10^{-3}$ & $0.5 \times 10^{-2}$ \\
\hline 20 & 8 & $151 \pm 2$ & $1.0 \pm 15$ & 24 & 211 & $80 \times 10^{-3}$ & $18 \times 10^{-2}$ \\
\hline 24 & 4 & $1.4 \pm 6$ & $0.7 \pm 10$ & 1.1 & 31 & $4 \times 10^{-3}$ & $0.8 \times 10^{-2}$ \\
\hline 26 & 4 & $1.9 \pm 7$ & $1.8 \pm 6$ & 1.8 & 3 & $6 \times 10^{-3}$ & $1.4 \times 10^{-2}$ \\
\hline 29 & 4 & $0.9 \pm 7$ & $0.6 \pm 10$ & 0.8 & 17 & $3 \times 10^{-3}$ & $0.6 \times 10^{-2}$ \\
\hline 30 & 4 & $1.1 \pm 7$ & $0.6 \pm 12$ & 0.8 & 28 & $3 \times 10^{-3}$ & $0.6 \times 10^{-2}$ \\
\hline 31 & 4 & $0.5 \pm 12$ & $0.4 \pm 14$ & 0.4 & 13 & $1 \times 10^{-3}$ & $0.3 \times 10^{-2}$ \\
\hline 32 & 4 & $0.8 \pm 9$ & $0.5 \pm 12$ & 0.7 & 20 & $2 \times 10^{-3}$ & $0.5 \times 10^{-2}$ \\
\hline 33 & 4 & $1.2 \pm 7$ & $0.7 \pm 10$ & 1 & 22 & $3 \times 10^{-3}$ & $0.8 \times 10^{-2}$ \\
\hline 34 & 3 & $1.1 \pm 7$ & $0.5 \pm 14$ & 0.8 & 37 & $3 \times 10^{-3}$ & $0.6 \times 10^{-2}$ \\
\hline
\end{tabular}

See Fig. 9.

${ }^{b} \mathrm{CG}=3 \times 10^{-3} \mu \mathrm{Cu} / \mathrm{ml}$. 
Table 19. Tritium (HTO) in water-Site 300.

\begin{tabular}{|c|c|c|c|c|c|c|c|}
\hline \multirow[b]{2}{*}{ Location" } & \multirow{2}{*}{$\begin{array}{l}\text { Number } \\
\text { of samples }\end{array}$} & \multicolumn{3}{|c|}{$10^{-8} \mu \mathrm{Ci} / \mathrm{ml} \pm 2 \sigma(\%)$} & \multirow[b]{2}{*}{$\%$ SDM } & \multirow[b]{2}{*}{$\% \mathrm{CG}^{\mathrm{b}}$} & \multirow{2}{*}{$\begin{array}{l}\text { Calculated } \\
\text { adult whole- } \\
\text { body dose, } \\
\text { mrem }\end{array}$} \\
\hline & & Maximum & Minimum & Average & & & \\
\hline 01 & 4 & $6.4 \pm 10$ & $29 \pm 22$ & 4.2 & 38 & $\times 10^{-3}$ & $3.1 \times 10^{-3}$ \\
\hline 02 & 4 & $3.1 \pm 22$ & $2 \pm 27$ & 2.6 & 18 & $\times 10^{-3}$ & $2 \times 10^{-3}$ \\
\hline 03 & 4 & $1.8 \pm 33$ & $0.4 \pm 100$ & 1.1 & 73 & $0.4 \times 10^{-3}$ & $0.8 \times 10^{-3}$ \\
\hline 94 & 4 & $1.9 \pm 34$ & $0.6 \pm 100$ & 1.2 & 45 & $0.4 \times 10^{-3}$ & $0.9 \times 10^{-3}$ \\
\hline 05 & 4 & $3.3 \pm 19$ & $1.5 \pm 41$ & 2.4 & 34 & $1 \times 10^{-3}$ & $1.8 \times 10^{-3}$ \\
\hline 06 & 4 & $1.5 \pm 40$ & $0.5 \pm 100$ & 1.0 & 44 & $0.3 \times 10^{-3}$ & $0.8 \times 10^{-3}$ \\
\hline 07 & 4 & $1.9 \pm 30$ & $0.5 \pm 100$ & 1.3 & 45 & $0.4 \times 10^{-3}$ & $1 \times 10^{-3}$ \\
\hline 11 & 4 & $6.2 \pm 10$ & $0.3 \pm 100$ & 3.2 & 84 & $1 \times 10^{-3}$ & $2.4 \times 10^{-3}$ \\
\hline 14 & 4 & $8.2 \pm 8$ & $4.8 \pm 14$ & 6.6 & 25 & $\times 10^{-3}$ & $5.1 \times 10^{-3}$ \\
\hline 20 & 8 & $14.5 \pm 7$ & $3.4 \pm 18$ & 8.4 & 46 & $\times 10^{-3}$ & $6.4 \times 10^{-3}$ \\
\hline 21 & 4 & $5.4 \pm 12$ & $1.6 \pm 29$ & 2.8 & 61 & $\times 10^{-3}$ & $2.1 \times 10^{-3}$ \\
\hline
\end{tabular}

"See Fig. 10.

${ }^{b} \mathrm{CG}=3.0 \times 10^{-3} \mu \mathrm{Cu} / \mathrm{ml}$.

Table 20. Tritium (HTO) in groundwater - Livermore Valley.

\begin{tabular}{|c|c|c|c|c|}
\hline Location" & $\begin{array}{c}\text { Well } \\
\text { identification }\end{array}$ & Well depth, m & $\begin{array}{c}\text { Activity } \\
10^{-6} \mu \mathrm{Ci} / \mathrm{ml} \pm 2 \sigma(\%)\end{array}$ & $\% \mathbf{C G}^{b}$ \\
\hline 1 & 3S1E-1P2 & 15 & 2.11 & $7.0 \times 10^{-2}$ \\
\hline 2 & 3S1E-2P3 & 116 & $0.007 \pm 77$ & $0.02 \times 10^{-2}$ \\
\hline 3 & 3S1E-2R1 & 10 & $1.26 \pm 3$ & $4.2 \times 10^{-2}$ \\
\hline 4 & 3S1E-3Q1 & 107 & $0.50 \pm 4$ & $1.7 \times 10^{-2}$ \\
\hline 5 & 3S1E-8H4 & 61 & $0.07 \pm 9$ & $0.23 \times 10^{-2}$ \\
\hline 6 & 3S1E-9G1 & 49 & $0.38 \pm 3$ & $1.3 \times 10^{-2}$ \\
\hline 7 & 3S1E-9M2 & 170 & $0.02 \pm 30$ & $0.07 \times 10^{-2}$ \\
\hline 8 & 3S1E-9P5 & 32 & $0.13 \pm 7$ & $0.43 \times 10^{-2}$ \\
\hline 9 & 3S1E-10A2 & 27 & $0.90 \pm 3$ & $3.0 \times 10^{-2}$ \\
\hline 10 & 3S1E-11B1 & 13 & $1.23 \pm 3$ & $4.1 \times 10^{-2}$ \\
\hline 11 & 3S1E-12D2 & 14 & $1.03 \pm 3$ & $3.4 \times 10^{-2}$ \\
\hline 12 & 3S1E-12G1 & 27 & $0.81 \pm 3$ & $2.7 \times 10^{-2}$ \\
\hline 13 & 3S1E-12N1 & 93 & $0.13 \pm 6$ & $0.43 \times 10^{-2}$ \\
\hline 14 & 3S1E-12P1 & 106 & $0.05 \pm 13$ & $0.17 \times 10^{-2}$ \\
\hline 15 & 3S1E-16H2 & 29 & $0.10 \pm B$ & $0.33 \times 10^{-2}$ \\
\hline 16 & 3S1E-16L7 & 197 & $0.05 \pm 12$ & $0.17 \times 10^{-2}$ \\
\hline 17 & 3S2E-7C2 & 15 & $0.19 \pm 5$ & $0.63 \times 10^{-2}$ \\
\hline 18 & $352 \mathrm{E}-7 \mathrm{~N} 1$ & 41 & $0.09 \pm 8$ & $0.30 \times 10^{-2}$ \\
\hline 19 & 3S2E-10Q1 & 13 & $0.14 \pm 6$ & $0.47 \times 10^{-2}$ \\
\hline 20 & 3S2E-14A3 & 34 & $0.06 \pm 11$ & $0.20 \times 10^{-2}$ \\
\hline
\end{tabular}

'See Fig. 11.

${ }^{b} \mathrm{CG}=3.0 \times 10^{-3} \mu \mathrm{Cl} / \mathrm{ml}$. 
Table 21. Tritium (HTO) in vegetation - Livermore Valley.

\begin{tabular}{|c|c|c|c|c|c|c|}
\hline \multirow[b]{2}{*}{ Location" } & \multirow{2}{*}{$\begin{array}{l}\text { Number } \\
\text { of samples }\end{array}$} & \multicolumn{3}{|c|}{$10^{-7} \mu \mathrm{Ci} / \mathrm{ml} \pm 2 \sigma(\%)$, water recovered } & \multirow[b]{2}{*}{$\%$ SDM } & \multirow{2}{*}{$\begin{array}{l}\text { adult whole- } \\
\text { body dose, } \\
\text { mrem }\end{array}$} \\
\hline & & Maximum & Minimum & Average & & \\
\hline 04 & 3 & $8.3 \pm 3$ & $1.1 \pm 100$ & 3.9 & 100 & $3.5 \times 10^{-2}$ \\
\hline 15 & 2 & $16.1 \pm 7$ & $7.1 \pm 17$ & 11.6 & 55 & $10.3 \times 10^{-2}$ \\
\hline 16 & 2 & $2 \pm 47$ & $1.1 \pm 100$ & 1.6 & 41 & $1.4 \times 10^{-2}$ \\
\hline 20 & 3 & $11.3 \pm 11$ & $5.3 \pm 19$ & 7.7 & 41 & $6.8 \times 10^{-2}$ \\
\hline 22 & 2 & $6.2 \pm 16$ & $4.1 \pm 29$ & 5.2 & 29 & $4.6 \times 10^{-2}$ \\
\hline 23 & 2 & $16.8 \pm 7$ & $11 \pm 12$ & 13.9 & 29 & $12.4 \times 10^{-2}$ \\
\hline 29 & 3 & $35 \pm 4$ & $7.4 \pm 17$ & 16.9 & 93 & $15 \times 10^{-2}$ \\
\hline 31 & 3 & $6.6 \pm 15$ & $3.6 \pm 32$ & 5.4 & 29 & $4.8 \times 10^{-2}$ \\
\hline 32 & 1 & $2.1 \pm 55$ & - & 2.1 & - & $1.9 \times 10^{-2}$ \\
\hline 33 & 1 & $1.1 \pm 100$ & - & 1.1 & - & $1 \times 10^{-2}$ \\
\hline
\end{tabular}

'See Fig. 12.

Table 22. Tritium (HTO) in vegetation - Site 300 .

\begin{tabular}{|c|c|c|c|c|c|c|}
\hline \multirow[b]{2}{*}{ Location" } & \multirow{2}{*}{$\begin{array}{l}\text { Number } \\
\text { of amples }\end{array}$} & \multicolumn{3}{|c|}{$10^{-7} \mu \mathrm{Ci} / \mathrm{ml} \pm 2 \sigma(\%)$, water recovered } & \multirow[b]{2}{*}{ \% SDM } & \multirow{2}{*}{$\begin{array}{l}\text { Calculated } \\
\text { adult whole- } \\
\text { body dose, } \\
\text { mrem }\end{array}$} \\
\hline & & Maximum & Minimum & Average & & \\
\hline 01 & 2 & $1.9 \pm 50$ & $1.4 \pm 79$ & 1.6 & 21 & $1.4 \times 10^{-2}$ \\
\hline 02 & 3 & $1.2 \pm 100$ & $1.1 \pm 100$ & 1.2 & 5 & $1.1 \times 10^{-2}$ \\
\hline 03 & 3 & $1.3 \pm 88$ & $1.1 \pm 100$ & 1.2 & 10 & $1.1 \times 10^{-2}$ \\
\hline 06 & 2 & $10.2 \pm 10$ & $5.3 \pm 6$ & 7.8 & 45 & $6.9 \times 10^{-2}$ \\
\hline 12 & 2 & $1.1 \pm 100$ & $0.9 \pm 100$ & 1 & 14 & $0.9 \times 10^{-2}$ \\
\hline 13 & 2 & $9.7 \pm 13$ & $2.3 \pm 41$ & 5.8 & 85 & $5.2 \times 10^{-2}$ \\
\hline
\end{tabular}

'See Fig. 13.

Table 23. Tritium (HTO) in wine.

\begin{tabular}{|c|c|c|c|c|c|}
\hline \multirow[b]{2}{*}{ Location } & \multirow{2}{*}{$\begin{array}{l}\text { Number } \\
\text { of samples }\end{array}$} & \multicolumn{3}{|c|}{$10^{-7} \mu \mathrm{Ci} / \mathrm{ml} \pm 2 \sigma(\%)$, water recovered } & \multirow[b]{2}{*}{ \% SDM } \\
\hline & & Maximum & Minimum & Average & \\
\hline Livermare Valley & 6 & $3.3 \pm 7$ & $0.8 \pm 24$ & 1.9 & 54 \\
\hline Other California areas & 2 & $0.5 \pm 26$ & $0.5 \pm 24$ & 0.5 & $\mathbf{0}$ \\
\hline Europe & 2 & $8.5 \pm 4$ & $2.2 \pm 7$ & 5.4 & 83 \\
\hline
\end{tabular}


Table 24. Tritium (HTO) in honey.

\begin{tabular}{|c|c|c|c|c|c|}
\hline \multirow[b]{2}{*}{ Location } & \multirow{2}{*}{$\begin{array}{c}\text { Number } \\
\text { of samples }\end{array}$} & \multicolumn{3}{|c|}{$10^{-6} \mu \mathrm{Ci} / \mathrm{ml} \pm 2 \sigma(\%)$, water recovered } & \multirow[b]{2}{*}{$\%$ SDM } \\
\hline & & Maximum & Minimum & Average & \\
\hline Livermore Valley & 2 & $0.43 \pm 6$ & $0.26 \pm 9$ & 0.35 & 35 \\
\hline Other California areas & 2 & $0.24 \pm 10$ & $0.14 \pm 15$ & 0.19 & 37 \\
\hline
\end{tabular}

Table 25. Various radionuclides in milk - Livermore Valley.

\begin{tabular}{|c|c|c|c|}
\hline & $\frac{10^{-7} \mu \mathrm{Ci} / \mathrm{ml} \pm 2 \sigma(\%)}{\mathrm{HTO}}$ & $\frac{10^{-6} \mu \mathrm{Ci} / \mathrm{ml} \pm 2 \sigma(\%)}{{ }^{40} \mathrm{~K}}$ & $\frac{10^{-9} \mu \mathrm{Ci} / \mathrm{ml} \pm 2 \sigma(\%)}{{ }^{137} \mathrm{Cs}}$ \\
\hline Number of samples & 18 & 17 & 17 \\
\hline Maximum & $111 \pm 2$ & $2.5 \pm 4$ & $4.5 \pm 36$ \\
\hline Minimum & $0.9 \pm 100$ & $1.6 \pm 4$ & $0.7 \pm 100$ \\
\hline Average & 7.9 & 1.9 & 2.4 \\
\hline$\%$ SDM & 327 & 12 & 45 \\
\hline $\begin{array}{l}\text { Calculated adult whole- } \\
\text { body dose, mrem }\end{array}$ & $3 \times 10^{-2}$ & 23 & $\times 10^{-2}$ \\
\hline
\end{tabular}


Table 26. Environmental radiation measurements (TLD) - LLNL perimeter.

\begin{tabular}{|c|c|c|c|c|c|}
\hline \multirow[b]{2}{*}{ Location" } & \multicolumn{5}{|c|}{ mrem } \\
\hline & Jan.-Mar. & Apr.-June & July-Sept. & Oct.-Dec. & Annual \\
\hline 1 & 15 & 13 & 15 & 14 & 57 \\
\hline 2 & 13 & 13 & 14 & 14 & 54 \\
\hline 3 & 15 & 13 & 16 & 16 & 60 \\
\hline 4 & 16 & 13 & 14 & 15 & 58 \\
\hline 5 & 16 & 14 & 16 & 15 & 61 \\
\hline 6 & 13 & 13 & 15 & 13 & 54 \\
\hline 7 & 13 & 13 & 15 & 13 & 54 \\
\hline 8 & 13 & lost & 14 & 15 & 56 \\
\hline 9 & 13 & 13 & 15 & 12 & 53 \\
\hline 10 & 13 & 12 & 12 & 13 & 50 \\
\hline 11 & 14 & 14 & 12 & 13 & 53 \\
\hline 12 & 21 & 12 & 12 & 12 & 57 \\
\hline 13 & 15 & 13 & 13 & 14 & 55 \\
\hline 42 & 14 & 12 & 12 & 13 & 51 \\
\hline 47 & 13 & 11 & 12 & 14 & 50 \\
\hline 48 & 14 & 11 & 12 & 13 & 50 \\
\hline 49 & 13 & 12 & 12 & 14 & 51 \\
\hline 50 & 14 & 11 & 13 & 12 & 50 \\
\hline 52 & 14 & 12 & 13 & 14 & 53 \\
\hline 53 & 14 & 12 & 13 & 13 & 52 \\
\hline 54 & 14 & 12 & 13 & 13 & 52 \\
\hline 56 & 13 & 13 & 12 & lost & 51 \\
\hline Average & 14 & 12 & 13 & $14 \ldots$ & 54 \\
\hline
\end{tabular}

¿See Fig. 14.

Table 27. Environmental neutron monitoring-LLNL perimeter.

\begin{tabular}{|c|c|c|c|c|c|}
\hline \multirow[b]{2}{*}{ Location" } & \multicolumn{5}{|c|}{ nrem } \\
\hline & Jan.-Mar. & Apr.-June & July-Sept. & Oct.-Dec. & Annual \\
\hline 1 & 2 & 0.5 & 0.7 & 1.2 & 4.4 \\
\hline 2 & 1.3 & 2.3 & 1.4 & 1 & 6 \\
\hline 3 & 22.5 & 3.4 & 3.6 & 3.7 & 33 \\
\hline 4 & 1.5 & 1 & 1.2 & 1.1 & 4.8 \\
\hline 5 & 2 & 1.4 & 1.5 & 1.3 & 6.2 \\
\hline 6 & 1.1 & 1.1 & 1.4 & 1 & 4.6 \\
\hline 7 & 1.3 & 1.2 & 1.6 & 1 & 5.1 \\
\hline 8 & 1.6 & 1.6 & 1.8 & 1.8 & 6.8 \\
\hline Average & 4.2 & 1.6 & 1.7 & 1.5 & 8.9 \\
\hline
\end{tabular}

See Fig. 3. 
Table 28. Beryllium on air filters - LLNL perimeter.

\begin{tabular}{|c|c|c|c|c|c|c|c|c|c|c|c|c|c|c|}
\hline \multirow[b]{2}{*}{ Location" } & \multicolumn{12}{|c|}{$10^{-5} \mu \mathrm{g} / \mathrm{m}^{3}$} & \multirow[b]{2}{*}{ Average } & \multirow[b]{2}{*}{$\%$ SDM } \\
\hline & Jan. & Feb. & Mar. & Apr. & May & June & July & Aug. & Sept. & Oct. & Nov. & Dec. & & \\
\hline 01 & $<1.9$ & $<3.0$ & $<1.6$ & 1.6 & 1.7 & 2 & $<34^{b}$ & 27 & $<36$ & 33 & 8 & 3.1 & $<14$ & 112 \\
\hline 02 & $<2.6$ & $<1.4$ & $<1.1$ & i.4 & 4.1 & 2 & $<46$ & 19 & $<35$ & 20 & 12 & 2.3 & $<12$ & 123 \\
\hline 12 & $<3.4$ & $<2.3$ & $<1.1$ & 1.9 & 5.3 & 3.3 & $<44$ & 29 & $<54$ & 26 & 22 & 4.9 & $<16$ & 110 \\
\hline 13 & $<1.7$ & $<1.4$ & $<1.1$ & $<1.4$ & 1.5 & 1.1 & $<23$ & 15 & $<17$ & 16 & 7.9 & 2.1 & $<7$ & 108 \\
\hline 14 & $<2.1$ & $<1.7$ & $<1.4$ & 1.9 & 1.8 & 1.5 & $<28$ & 25 & $<22$ & 25 & 8.7 & 1.6 & $<10$ & 112 \\
\hline 15 & $<1.5$ & $<1.6$ & $<1.1$ & 1.4 & 4.9 & 3.5 & 24 & 30 & $<18$ & 1.1 & 9.2 & 1.1 & $<B$ & 123 \\
\hline Average & $<2.2$ & $<1.9$ & $<1.2$ & $<1.6$ & 3.2 & 2.2 & $<33$ & 24 & $<30$ & 20 & 11 & 2.5 & $<11$ & \\
\hline$\%$ SDM & 32 & 33 & 18 & 15 & 54 & 43 & 30 & 25 & 47 & 54 & 48 & 54 & & \\
\hline \% Standard ${ }^{c}$ & $=0.2$ & $<0.2$ & $<0.1$ & $<0.2$ & 0.3 & 0.2 & $<3$ & 2 & $<3$ & 2 & 1 & 0.2 & $<1.1$ & \\
\hline
\end{tabular}

See Fig. 3.

The variations in detection limits are due to the different types of instrumentation and techniques used for the analyses.

Emission standard is $0.01 \mu \mathrm{g} / \mathrm{m}^{3}$. 
Table 29. Beryllium on air filters - Site 300 .

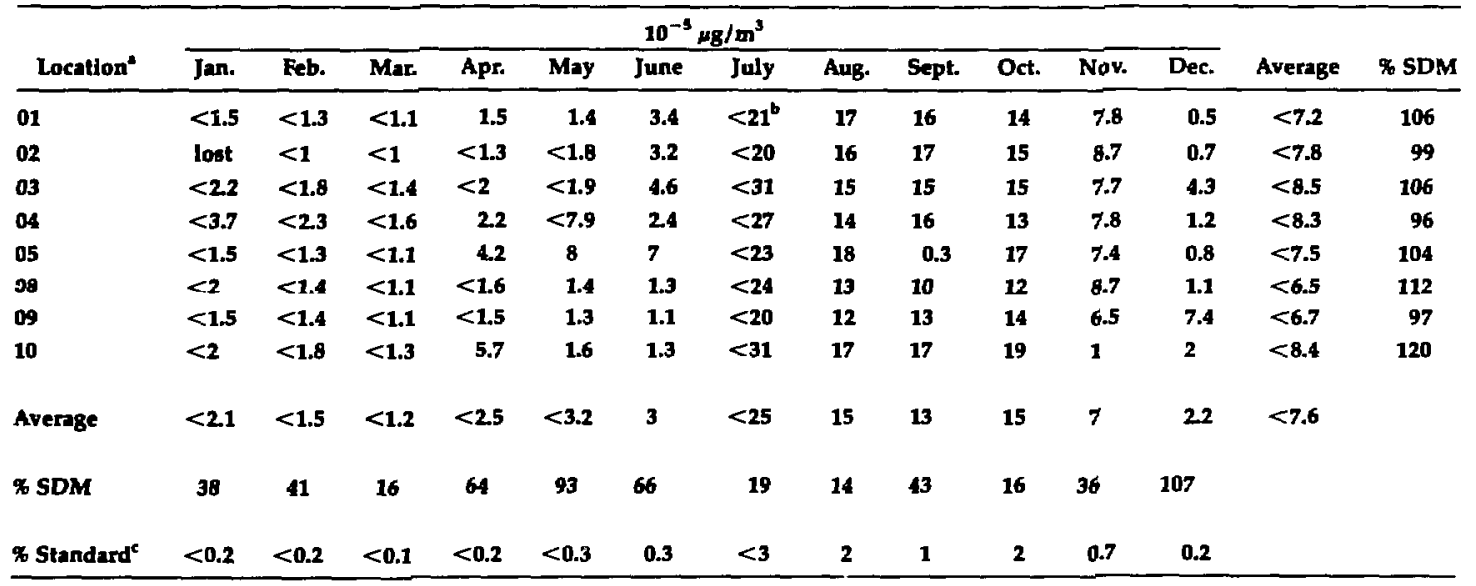

See Fig. 4.

bhe variations in detection limits are due to the different instrumentation and techniques used for the analyses.

'Emisoion standard is $0.01 \mathrm{mg} / \mathrm{m}^{3}$. 
Table 30. Various elements in LLNL liquid effluent.

\begin{tabular}{|c|c|c|c|c|c|c|c|c|}
\hline \multirow[b]{2}{*}{ Month } & \multicolumn{8}{|c|}{ pron } \\
\hline & Cd & $\mathrm{Cr}$ & $\mathrm{Cu}$ & $\mathbf{F e}$ & $\mathbf{P b}$ & $\mathbf{N i}$ & $\overline{\mathbf{A}_{\mathbf{B}}}$ & $\mathbf{Z n}_{\mathbf{n}}$ \\
\hline Jan. & 0.002 & 0.29 & 0.16 & 1.03 & 0.01 & 0.06 & 0.17 & 0.26 \\
\hline Feb. & 0.005 & 0.25 & 0.31 & 1.04 & 0.02 & 0.06 & 0.11 & 0.23 \\
\hline Mar. & 0.004 & 0.35 & 0.23 & 1.16 & 0.04 & 0.16 & 0.27 & 0.34 \\
\hline Apr. & 0.001 & 0.24 & 0.13 & 0.86 & 0.03 & 0.02 & 0.18 & D.30 \\
\hline May & 0.002 & 0.20 & 0.16 & 0.64 & 0.01 & 0.09 & 0.03 & 0.20 \\
\hline June & 0.001 & 0.14 & 0.13 & 0.64 & 0.03 & 0.07 & 0.11 & 0.23 \\
\hline July & 0 & 0.08 & 0.08 & 0.42 & 0.02 & 0.02 & 0.09 & 0.16 \\
\hline Aug. & 0.002 & 0.16 & 0.21 & 0.58 & 0.02 & 0.03 & 0 & 0.35 \\
\hline Sept. & 0.001 & 0.24 & 0.12 & 0.84 & 0.02 & 0.02 & 0.01 & 0.25 \\
\hline Oct. & 0.002 & 0.22 & 0.16 & 0,92 & 0.01 & 0.02 & 0.05 & 0.32 \\
\hline Nov. & 0.001 & 0.19 & 0.15 & 1.1 & 0.01 & 0.01 & 0.09 & 0.4 \\
\hline Dec. & 0.001 & 0.17 & 0.12 & 0.88 & 0.01 & 0.01 & 0.05 & 0.33 \\
\hline Average & 0.002 & 0.21 & 0.16 & 0.84 & 0.02 & 0.13 & 0.10 & 0.28 \\
\hline$\%$ SDM & 76 & 34 & 37 & 27 & 52 & 213 & 82 & 25 \\
\hline
\end{tabular}


Table 31. Physical and chemical examinations of LLNL liquid effluent.

\begin{tabular}{|c|c|c|c|c|c|c|}
\hline \multirow[b]{2}{*}{ Analysis } & \multicolumn{5}{|c|}{ mg/liter } & \multirow[b]{2}{*}{$\%$ SDM } \\
\hline & Jan.-Mar. & Apr. $\rightarrow$ Jr je & July-Sept. & Oct.-Dec. & Average & \\
\hline BOD & 120 & $\mathbf{5 0}$ & 67 & 59 & 74 & 42 \\
\hline COD & 194 & 2050 & 150 & 132 & 632 & 150 \\
\hline $\begin{array}{l}\text { Total nitrogen } \\
\text { (as N) }\end{array}$ & 30 & 24 & 28 & 27 & 27 & 9 \\
\hline Ammonia nitrogen & 24 & 19 & 21 & 23 & 22 & 10 \\
\hline Nitrate nitrogen & 0.07 & 0.03 & 0.028 & 0.01 & 0.03 & 72 \\
\hline $\begin{array}{l}\text { Nitrite nitrogen } \\
\text { (as N) }\end{array}$ & $<0.01$ & $<0.01$ & $<0.01$ & 0.015 & $<0.01$ & 22 \\
\hline $\begin{array}{l}\text { Oil and grease } \\
\text { (FEM) }\end{array}$ & 15 & $<5$ & 10 & 38 & $<17$ & 86 \\
\hline Sulfate & 39 & 51 & 46 & 54 & 48 & 14 \\
\hline Arsenic & $<0.0005$ & 0.0034 & 0.0043 & 0.0078 & $<0.0040$ & 75 \\
\hline Boron & 0.58 & 0.51 & 0.42 & 0.64 & 0.54 & 18 \\
\hline Cyanide & 0.16 & 0.28 & 0.57 & 0.52 & 0.38 & $\mathbf{5 1}$ \\
\hline Mersury & 0.0036 & 0.0008 & 0.0012 & 0.0007 & 0.0016 & 87 \\
\hline Selerium & $<0.0005$ & $<0.0005$ & $<0.0005$ & $<0.0005$ & $<0.0005$ & \\
\hline Total alkalinity & 118 & 44 & 99 & 92 & 88 & 36 \\
\hline \multicolumn{7}{|l|}{ Total phosphorus } \\
\hline (as P) & 5.3 & 3.9 & 4.8 & 4.2 & 4.6 & 14 \\
\hline Chloride & 3 & 25 & 34 & 52 & 28 & 71 \\
\hline Phenols & 0.065 & $<0.005$ & 0.020 & 0.010 & $<0.025$ & 110 \\
\hline Calcium & 8.0 & 13 & 10 & 7.5 & 9.6 & 26 \\
\hline Magnesium & 1.6 & 0.11 & 1.8 & 2.2 & 1.4 & 64 \\
\hline Sodiun & 31 & 20 & 27 & 36 & 28 & 24 \\
\hline Chrnmium & 0.16 & 0.11 & 0.18 & 0.17 & 0.16 & 20 \\
\hline Copper & 0.094 & 0.046 & 0.10 & 0.05 & 0.07 & 39 \\
\hline Nickel & 0.30 & 0.069 & 0.022 & $<0.04$ & 0.11 & 120 \\
\hline Potassium & 16 & 15 & 17 & 15 & 16 & 6 \\
\hline Zinc & 0.19 & 0.19 & 0.28 & 0.35 & 0.25 & 31 \\
\hline $\begin{array}{l}\text { Sodium as \% } \\
\text { of cations }\end{array}$ & 57 & 46 & 16 & 70 & 47 & 49 \\
\hline Total solids & $\mathbf{2 7 5}$ & 219 & 232 & 289 & 254 & 13 \\
\hline DissnIved solids & 247 & 181 & 182 & 236 & 212 & 16 \\
\hline $\begin{array}{l}\text { Volatile solids } \\
\text { as } \% \text { of total solids }\end{array}$ & 40 & 38 & 37 & 30 & 36 & 12 \\
\hline $\begin{array}{l}\text { Settleable solids } \\
\text { (ml/liter/h) }\end{array}$ & 1.8 & $<0.5$ & 1.9 & 1.5 & 1.4 & 45 \\
\hline $\begin{array}{l}\text { Identifiable chlorinated } \\
\text { hydrocarbons }\end{array}$ & $<\operatorname{LOS}^{\prime}$ & $<\operatorname{LOS}$ & $<\operatorname{LOS}$ & $\begin{array}{l}\text { le, } 0.26 \mu \mathrm{g} / \mathrm{lit} \\
\mathrm{DD}, 0.07 \mu \mathrm{g} / 1\end{array}$ & & \\
\hline PCB & $<\operatorname{LOS}$ & $<\operatorname{LOS}$ & $<\operatorname{LOS}$ & $<\operatorname{LOS}$ & & \\
\hline
\end{tabular}

"The following compounds would have been reported had they appeared at or above their respective detection limito as indicated below:

\begin{tabular}{|c|c|c|c|c|c|}
\hline Organochlorine pesticides & $\mu g / l i t e r$ & Organochlorine pesticides & mg/liter & Polychlorinated biphenyls & $\mu \mathrm{g} /$ liter \\
\hline Aldrin & 0.05 & Dieldrin & 0.05 & Aroclors 1016-1262 & 0.3 \\
\hline ВHC & 0.05 & Endrin & 0.1 & & \\
\hline Lindane & 0.05 & Heptachlor & 0.05 & & \\
\hline Chlordane & 0.1 & Heptachlor epoxide & 0.05 & & \\
\hline DDD & 0.05 & Methoxychlor & 0.2 & & \\
\hline DDE & 0.05 & Toxaphene & 1 & & \\
\hline
\end{tabular}


Table 32. Site $\mathbf{3 0 0}$ water samples down gradient from liquid-discharge sites.

\begin{tabular}{|c|c|c|c|c|}
\hline \multirow[b]{2}{*}{ Locations" } & \multirow[b]{2}{*}{$\begin{array}{l}\text { Specific conductance, } \\
\mu m h o s / \mathrm{cm} \text { at } 25^{\circ} \mathrm{C}\end{array}$} & \multicolumn{3}{|c|}{ mg/liter } \\
\hline & & $\begin{array}{c}\text { Tolal hardness } \\
\left.\text { (as } \mathrm{CaCO}_{3}\right)\end{array}$ & Chloride & Total organic carbon \\
\hline 3 Jan. & 797 & 83 & 61 & 20.2 \\
\hline Apr. & 790 & 80 & 60 & 13.6 \\
\hline 4 Jan. & 1200 & 15 & 151 & 5.5 \\
\hline Apr. & 1220 & 18 & 154 & 4.3 \\
\hline Oct. & 1230 & 24 & 170 & 29 \\
\hline 5 Jan. & 1000 & 70 & 77 & 4.7 \\
\hline Apr. & 1000 & 68 & 77 & 5.7 \\
\hline Oct. & 760 & 64 & 70 & 27 \\
\hline 14 Jan. & 1950 & 508 & 164 & 33 \\
\hline Apr. & 1940 & 461 & 173 & 8.8 \\
\hline Oct. & 2760 & 970 & 215 & 44 \\
\hline 8 Oct. & 670 & 88 & 64 & 24 \\
\hline
\end{tabular}

'See Fig. 10.

Table 33. Estimated radiation dose to the public from LLNL airborne effluents during 1981.

\begin{tabular}{|c|c|c|c|c|c|}
\hline Nuclide & Facility & Curies & $\begin{array}{l}\text { Dose at site } \\
\text { boundary, } \\
\text { mrem }\end{array}$ & $\begin{array}{l}\text { Dose to nearest } \\
\text { resident, } \\
\text { mrem }\end{array}$ & $\begin{array}{c}\text { Dose within } 80 \mathrm{~km} \\
\text { iadius of LLNL, } \\
\text { man-rem }\end{array}$ \\
\hline \multirow[t]{3}{*}{${ }^{3} \mathbf{H}$} & Tritium facility & 2552 & 0,33 & 0.26 & 1.5 \\
\hline & $\begin{array}{l}\text { Insulating } \\
\text { core accelerator }\end{array}$ & 23 & 0.02 & 0.02 & 0.01 \\
\hline & $\begin{array}{l}\text { Rotating target } \\
\text { neutron source }\end{array}$ & 44 & 0.02 & 0.01 & 0.03 \\
\hline${ }^{13} N-{ }^{15} \mathrm{O}$ & Linear accelerator & 344 & 0.50 & 0.13 & 0.03 \\
\hline
\end{tabular}


Table 34. Radioactive effluent releases from LLNL from 1976 through 1981.

\begin{tabular}{|c|c|c|c|c|c|c|}
\hline \multirow{3}{*}{$\frac{\text { Year }}{1976}$} & \multicolumn{3}{|c|}{ Airborne effuents, $\mathrm{Ci}$} & \multicolumn{3}{|c|}{ Liquid effluents, $\mathrm{Ci}$} \\
\hline & ${ }^{41} \mathrm{Ar}$ & ${ }^{\mathbf{3}} \mathbf{H}$ & ${ }^{13} \mathrm{~N}-{ }^{15} \mathrm{O}$ & HTO & & ${ }^{5 \mathbf{P u}}$ \\
\hline & 470 & 3991 & 1035 & 10 & 1.5 & $\times 10^{-4}$ \\
\hline 1977 & 380 & $\mathbf{5 2 1 0}$ & 990 & 13 & 3.6 & $\times 10^{-4}$ \\
\hline 1978 & 766 & 5362 & 1445 & 9 & 8.6 & $\times 10^{-4}$ \\
\hline 1979 & 383 & 4517 & 829 & 7 & 9.8 & $\times 10^{-4}$ \\
\hline 1980 & 165 & 2305 & 1656 & 5 & 28 & $\times 10^{-4}$ \\
\hline 1981 & o & 2620 & 344 & 6 & 0.92 & $\times 10^{-4}$ \\
\hline
\end{tabular}

Table 35. LLNL nonradiuactive liquid effluent - storm drain runoff.

\begin{tabular}{|c|c|c|c|c|}
\hline \multirow[b]{3}{*}{ Analyses } & \multicolumn{4}{|c|}{ mg/liter } \\
\hline & \multicolumn{2}{|c|}{ First major rain } & \multicolumn{2}{|c|}{ Second } \\
\hline & In & Out & In & Out \\
\hline Nitrate nitrogen (as $\mathrm{N}$ ) & 5.1 & 2.6 & 3.9 & 1 \\
\hline Total phosphorus (as P) & 1.7 & 0.9 & 0.8 & 0.5 \\
\hline Cyanide & 0.04 & $<0.02$ & $<0.02$ & $<0.0 \hat{2}$ \\
\hline Phenols & $<0.001$ & 0.002 & $<0.005$ & $<0.005$ \\
\hline $\begin{array}{l}\text { Oil and grease } \\
\text { (freon extractable) }\end{array}$ & 6.9 & $<5$ & 5 & $<5$ \\
\hline Sulfactants (MBAS) & 0.03 & $<0.02$ & & \\
\hline Sulfate & 11 & 4.4 & 2 & 14 \\
\hline $\begin{array}{l}\text { Total identifiable } \\
\text { chlorinated hydrucarbons }\end{array}$ & $\begin{array}{l}0.12 \mathrm{ppb} \\
\text { Lindane }\end{array}$ & None" & None" & None" \\
\hline
\end{tabular}

tThe following compounds would have been reported had they appeared at or above their respective detection limits as indicated below:

\begin{tabular}{ll} 
Organochlorine pesticides & $\mu \mathrm{g} /$ liter \\
\cline { 2 - 2 } Aldrin & 0.05 \\
BHC (mixed isomers) & 0.05 \\
Lindane & 0.05 \\
Chlordane & 0.1 \\
DDD & 0.05 \\
DDE & 0.05 \\
DDT & 0.1 \\
Dieldrin & 0.05 \\
Endrin & 0.1 \\
Heptachlor & 0.05 \\
Heptachlor epoxide & 0.05 \\
Methoxychlor & 0.2 \\
Toxaphene & 1
\end{tabular}

Polychlorinated blphenyls Aroclors 1016-1262 
Table 36. Summary of analyses for the Quality Assurance Program."

\begin{tabular}{|c|c|c|c|c|c|c|c|}
\hline Isotopp & Media & Period & LLNL & EML & All & LLNL/EML & LLNL/All \\
\hline \multirow[t]{2}{*}{${ }^{7} \mathrm{Be}$} & Air & $\mathbf{1}$ & 0.312 & 0.244 & 0.284 & 1.28 & 1.10 \\
\hline & & 2 & 1.025 & 0.590 & 0.630 & 1.74 & 1.63 \\
\hline \multirow[t]{2}{*}{${ }^{125} \mathrm{Sb}$} & Air & 1 & 0.183 & 0.139 & 0.157 & 1.32 & 1.17 \\
\hline & & 2 & 0.136 & 0.104 & 0,090 & 1.31 & 1.51 \\
\hline${ }^{137} \mathrm{Cs}$ & Air & 2 & 0.324 & 0.175 & 0.194 & 1.85 & 1.67 \\
\hline${ }^{208} \mathbf{U}$ & Air & 2 & 0.669 & 0.490 & 0.735 & 1.37 & 0.91 \\
\hline$U(\mu g)$ & Air & 1 & 0.194 & 0.223 & 0.222 & 0.87 & 0.87 \\
\hline${ }^{236} \mathrm{Pu}$ & Air & 1 & 0.343 & 0.370 & 0.340 & 0.93 & 1.01 \\
\hline \multirow[t]{2}{*}{${ }^{239} \mathrm{Pu}$} & Air & 1 & 0.510 & 0.560 & 0.530 & 0.91 & 0.96 \\
\hline & & 2 & 0.134 & 0.130 & 0.135 & 1.03 & 0.99 \\
\hline \multirow[t]{2}{*}{${ }^{3} \mathbf{H}$} & Water & 1 & 0.231 & 0.246 & 0.242 & 0.94 & 0.95 \\
\hline & & 2 & 0.156 & 0.156 & 0.167 & 1.00 & 0.93 \\
\hline${ }^{137} \mathrm{Cs}$ & Water & 1 & 0.131 & 0.137 & 0.139 & 0.96 & 0.94 \\
\hline${ }^{144} \mathrm{Ce}$ & Water & 2 & 0.114 & 0.113 & 0.106 & 1.01 & 1.08 \\
\hline \multirow[t]{2}{*}{${ }^{239} \mathrm{Pu}$} & Water & 1 & 0.491 & 0.550 & 0.500 & 0.89 & 0.98 \\
\hline & & 2 & 0.302 & 0.330 & 0.290 & 0.92 & 1.04 \\
\hline \multirow[t]{2}{*}{${ }^{10} \mathrm{~K}$} & Soil & 1 & 0.234 & 0.213 & 0.235 & 1.10 & 1.00 \\
\hline & & 2 & 0.194 & 0.200 & 0.192 & 0.97 & 1.01 \\
\hline \multirow[t]{2}{*}{${ }^{137} \mathrm{Cs}$} & Soil & $\mathbf{1}$ & 0.260 & 0.200 & 0.226 & 1.30 & 1.15 \\
\hline & & 2 & 0.496 & 0.419 & 0.407 & 1.18 & 1.22 \\
\hline${ }^{278} \mathrm{U}$ & Soil & 2 & 1.20 & 0.620 & 0.738 & 1.94 & 1.63 \\
\hline \multirow[t]{2}{*}{${ }^{238} \mathbf{P u}$} & Soll & 1 & 0.500 & 1.40 & 0.930 & 0.36 & 0.54 \\
\hline & & 2 & 0.222 & 0.250 & 0.252 & 0.89 & 0.88 \\
\hline \multirow[t]{2}{*}{${ }^{239} \mathrm{Pu}$} & Soil & $\mathbf{1}$ & 0.640 & 0.720 & 0.757 & 0.89 & 0.85 \\
\hline & & 2 & 0.817 & 0.770 & 0.823 & 1.06 & 0.99 \\
\hline \multicolumn{8}{|c|}{ Nonradioactive elements } \\
\hline \multirow[t]{2}{*}{$\mathrm{Cu}$} & Air & $\mathbf{1}$ & 0.102 & 0.102 & 0.101 & 1.00 & 1.01 \\
\hline & & 2 & 0.773 & 0.700 & 0.664 & 1.10 & 1.16 \\
\hline \multirow[t]{2}{*}{$\mathbf{P b}$} & Air & 1 & 0.170 & 0.204 & 0.190 & 0.83 & 0.89 \\
\hline & & 2 & 0.178 & 0.200 & 0.179 & 0.89 & 0.99 \\
\hline \multirow[t]{2}{*}{$\mathbf{Z n}$} & Air & 1 & 0.140 & 0.102 & 0.127 & 1.37 & 1.10 \\
\hline & & 2 & 0.472 & 0.500 & 0.583 & 0.94 & 0.81 \\
\hline $\mathrm{Cu}$ & Water & 2 & 0.391 & 0.380 & 0.379 & 1.03 & 1.03 \\
\hline \multirow[t]{2}{*}{$\mathbf{P b}$} & Water & 1 & 0.357 & 0.323 & 0.305 & 1.11 & 1.17 \\
\hline & & 2 & 0.108 & 0.109 & 0.107 & 0.99 & 1.01 \\
\hline \multirow[t]{2}{*}{$\mathbf{Z n}$} & Water & 1 & 0.153 & 0.161 & 0.164 & 0.95 & 0.93 \\
\hline & & 2 & 0.269 & 0.271 & 0.260 & 0.99 & 1.03 \\
\hline
\end{tabular}

"All of the values shown are relative; i.e., the exponents are not included, and therefore do not indicate lotal activity or concentrations. 


\section{Appendix B. Environmental Activity Concentration-Guide Levels}

The standards for Radiation Protection (DOE Manual 5480.1 Chg 2, issued April 29, 1981) state that the average activity of a mixture of radionuclides (whose identities and concentrations are unknown) in air and water should not exceed the following values:

1. Air (controlled area) $6 \times 10^{-13} \mu \mathrm{Ci} / \mathrm{ml}$

2. Air (uncontrolled area) $2 \times 10^{-14} \mu \mathrm{Ci} / \mathrm{ml}$

3. Water (controlled area) $4 \times 10^{-7} \mu \mathrm{Ci} / \mathrm{ml}$

4. Water (uncontrolled area) $3 \times 10^{-8} \mu \mathrm{Ci} / \mathrm{ml}$

If alpha emitters and ${ }^{227} \mathrm{Ac}$ are definitely not present, the following values may be used to determine permissible average activity:

5. Air (controlled area)

$3 \times 10^{-11} \mu \mathrm{Ci} / \mathrm{ml}$

6. Air (uncontrolled area)

$1 \times 10^{-12} \mu \mathrm{Ci} / \mathrm{ml}$

If ${ }^{129} \mathrm{I},{ }^{226} \mathrm{Ra}$, and ${ }^{228} \mathrm{Ra}$ are definitely not present, the following values may be used:

7. Water (controlled area) $3 \times 10^{-6} \mu \mathrm{Ci} / \mathrm{ml}$

8. Water (uncontrolled area) $1 \times 10^{-7} \mu \mathrm{Ci} / \mathrm{ml}$

Both air and water samples are subjected to gross alpha and gross beta measurements. The average annual alpha activities of samples may not exceed the activity values listed as 1-4 above. Since the alpha emitters have been accounted for in the gross alpha measurements and the assumption is made that ${ }^{129} \mathrm{I}$, ${ }^{227} \mathrm{Ac}$, ${ }^{226} \mathrm{Ra}$, and ${ }^{228} \mathrm{Ra}$ are not present in the samples, the average annual gross beta activities of the samples may not exceed the activities listed as 5-8 above. The assumption that ${ }^{129} \mathrm{I},{ }^{227} \mathrm{Ac},{ }^{226} \mathrm{Ra}$, and ${ }^{228} \mathrm{Ra}$ are not present in air and water samples is reasonable in view of the minute quantities of these radionuclides available at the Laboratory. This reference also states that average tritium activities in off-site water samples may not exceed $3 \times 10^{-3} \mu \mathrm{Ci} / \mathrm{ml}$.

The external whole-body radiation dose to workers in controlled areas may not exceed $5 \mathrm{rem} / \mathrm{y}$, and the dose to an individual in an uncontrolled area may not exceed $500 \mathrm{mrem} / \mathrm{y}$. Also, a group of individuals in an uncontrolled area may not receive an average annual dose of more than $\mathbf{1 7 0}$ mrem. 


\section{Appendix C. Method of Dose Calculations}

The doses shown in this report have been calculated using the models and methods in the Nuclear Regulatory Commission Regulatory Guide 1.109, Calculation of Annual Doses to Man from Routine Releases of Reactor Effluent. Examples of these calculations and assumptions are shown in this appendix.

\section{Annual Dose from Potable Water}

Assuming that all water sampled is available as drinking water, the annual whole-body dose for tritium has been calculated using the following equation:

$R_{\text {total body }}=C_{w} U_{w} D_{w}$.

where

$\mathrm{C}_{\mathrm{w}}=$ concentration in $\mathrm{pCi} /$ liter

$\mathrm{U}_{\mathrm{w}}=$ intake rate, liters/y,

$=730$ liters $/ y$ for maximum exposed individual,

$\mathrm{D}_{\mathrm{w}}=$ dose factor, mrem $/ \mathrm{pCi}$.

$=1.05 \times 10^{-7} \mathrm{mrem} / \mathrm{pCi}$ for the whole-body ingestion pathway for an adult.

$\mathrm{R}_{\text {total body }}=$ annual dose in mrem to the total body from ingestion of 730 liters of potable water with concentration $\mathrm{C}_{\mathrm{w}}$.

\section{Annual Dose from Forage-Cow-Milk Pathway for Tritium in Vegetation}

Assuming that all feed for the cattle was pasture grass, the annual whole-body dose per $\mu \mathrm{Ci} / \mathrm{ml} \mathrm{HTO}$ for the maximum exposed individual has been calculated using the following equation:

$D_{\text {total body }}=D_{\text {veg }}+D_{\text {meat }}+D_{\text {milk }}$.

$D_{\text {veg }}$ (leafy vegetables) $=U_{\text {veg }} \times C_{\text {veg }} \times D_{\text {HTO }}$.

where

$$
\begin{aligned}
& \mathrm{U}_{\text {veg }}=\text { intake rate, } \mathrm{kg} / \mathrm{y}, 64 \mathrm{~kg} / \mathrm{y} \text { for maximum exposed individual , } \\
& \mathrm{C}_{\mathrm{veg}}=\text { concentration in } \mathrm{pCi} / \mathrm{kg}=\frac{\mathrm{Pci} / \mathrm{kg}}{\mu \mathrm{Ci} / \mathrm{ml}} \times \mathrm{C}_{\mathrm{veg}} \mu \mathrm{Ci} / \mathrm{ml} \text { (measured) , } \\
& \begin{aligned}
& \mathrm{D}_{\mathrm{HTO}}= \text { dose factor, } \mathrm{mrem} / \mathrm{pCi}=1.05 \times 10^{-7} \mathrm{mrem} / \mathrm{pCi} \text { for }{ }^{3} \mathrm{H} \text { for the adult whole-body ingestion } \\
& \text { pathway. }
\end{aligned} \\
& D_{\text {meat }}=U_{\text {meat }} \times C_{\text {meat }} \times D_{\text {HTO }} \text { ， }
\end{aligned}
$$

where

$$
\begin{aligned}
& \mathrm{U}_{\text {meat }}=110 \mathrm{~kg} / \mathrm{y}_{1} \\
& \mathrm{D}_{\text {HTO }}=1.05 \times 10^{-7} \mathrm{mrem} / \mathrm{pCi}, \\
& C_{\text {meat }}=\left(F_{f}\right)\left(Q_{f}\right)\left(C_{\text {veg }}\right) \exp \left(-\lambda_{i} t_{s}\right) .
\end{aligned}
$$




$$
\begin{aligned}
& \mathrm{F}_{\mathrm{f}}=\text { fraction of daily intake of nuclide per } \mathrm{kg} \text { of animal } / \mathrm{fish} \text {, days } / \mathrm{kg}, \\
& \mathrm{Q}_{\mathrm{f}}=\text { amount of feed consumed, } \mathrm{kg} / \text { day, } \\
& \mathrm{C}_{\text {meat }}=\left(1.2 \times 10^{-2} \frac{\mathrm{day}}{\mathrm{kg}}\right)\left(50 \frac{\mathrm{kg}}{\mathrm{day}}\right)\left(3 \times 10^{9} \frac{\mathrm{pCi} / \mathrm{kg}}{\mu \mathrm{Ci} / \mathrm{ml}}\right) \\
& \quad \times \exp \left[-4.5 \times 10^{-3}(20)\right]=1.8 \times 10^{-9} \frac{\mathrm{pCi} / \mathrm{kg}}{\mu \mathrm{Ci} / \mathrm{ml}} \times \mathrm{C}_{\mathrm{veg}} \frac{\mu \mathrm{Ci}}{\mathrm{ml}} \text { (measured) } \\
& \mathrm{C}_{\mathrm{veg}}=\text { same as above, } \\
& \lambda_{\mathrm{i}}=\text { radiological decay constant, day }{ }^{-1}, \\
& \mathrm{t}_{\mathrm{s}}=\text { time between slaughter to consumption, days. }
\end{aligned}
$$

$\mathrm{C}_{\mathrm{veg}}=$ same as above,

$\mathrm{D}_{\text {milk }}=\mathrm{U}_{\text {milk }} \times \mathrm{C}_{\text {milk }} \times \mathrm{D}_{\mathrm{HTO}}$

where

$$
\begin{aligned}
& \mathrm{U}_{\text {milk }}=310 \text { liters } / y \text {, } \\
& \mathrm{D}_{\mathrm{HTO}}=1.05 \times 10^{-7} \mathrm{mrem} / \mathrm{pCi} \text {, } \\
& C_{\text {milk }}=F_{m} Q_{i} C_{v e g} \exp \left(-\lambda_{j} t_{f}\right) \text {, } \\
& \mathrm{F}_{\mathrm{m}}=\text { fraction of daily intake of nuclide per liter of milk, day/liter, } \\
& \mathrm{Q}_{\mathrm{f}}=\text { amount of feed consumed, } \mathrm{kg} / \text { day, } \\
& \mathrm{C}_{\text {veg }}=\text { same as above, } \\
& \lambda_{\mathrm{i}}=\text { radiological decay constant, days }{ }^{-1} \text {, } \\
& t_{\mathrm{f}}=\text { transport time from the feed to milk receptor, } \\
& C_{\text {milk }}=\left(1.0 \times 10^{-2} \frac{\text { day }}{\text { liter }}\right)\left(50 \frac{\mathrm{kg}}{\mathrm{d}}\right)\left(3 \times 10^{9} \frac{\mathrm{pCliter} / \mathrm{kg}}{\mu \mathrm{Ci} / \mathrm{ml}}\right) \\
& \times \exp \left[-4.5 \times 10^{3}(2)\right]=1.5 \times 10^{9} \frac{\mathrm{pCi} / \mathrm{liter}}{\mu \mathrm{Ci} / \mathrm{ml}} \times \mathrm{C}_{\mathrm{veg}} \frac{\mu \mathrm{Ci}}{\mathrm{ml}} \text { (measured) } . \\
& \mathrm{D}_{\text {total }}=\left(2.04 \times 10^{4} \frac{\mathrm{mrem}}{\mu \mathrm{Ci} / \mathrm{ml}}\right) \text { veg }+\left(2.07 \times 10^{4} \frac{\text { mrem }}{\mu \mathrm{Ci} / \mathrm{ml}}\right) \text { meat } \\
& +\left(4.88 \times 10^{4} \frac{\text { mrem }}{\mu \mathrm{Ci} / \mathrm{ml}}\right) \text { milk }=8.96 \times 10^{4} \frac{\text { mrem }}{\mu \mathrm{Ci} / \mathrm{ml}} \times \mathrm{C}_{\mathrm{veg}} \frac{\mu \mathrm{Ci}}{\mathrm{ml}} \text { (measured) }
\end{aligned}
$$

\section{Annual Population Dose}

To calculate the "fence-line" site boundary, nearest resident, and population doses, a continuouspoint-source computer code based on the Gaussian plume model was used.

$C_{\text {downwind }}=3.17 \times 10^{4}(\chi / Q)(Q)$, 
where

$$
\begin{aligned}
& \chi / Q=\text { diffusion parameter, sec } / \mathrm{m}^{3} \\
& \mathrm{Q}=\text { release rate, } \mathrm{Ci} / \mathrm{y}, \\
& 3.17 \times 10^{4}=1 \times 10^{12} \frac{\mathrm{pCi}}{\mathrm{Ci}} / 3.15 \times 10^{7} \frac{\mathrm{sec}}{\mathrm{y}} \\
& \mathrm{C}_{\text {downwind }}=\text { concentration downwind, } \mathrm{pCi} / \mathrm{m}^{3} \\
& \mathrm{D}_{\max \text { individual }}=\mathrm{U} \times \mathrm{C}_{\text {downwind }} \times \mathrm{D}
\end{aligned}
$$

where

$$
\begin{aligned}
& \mathrm{U}=\text { intake rate, } \mathrm{m}^{3} / \mathrm{y}=8000 \mathrm{~m}^{3} / \mathrm{y} \text {, inhalation rate } \\
& \text { for adult maximum exposed individual } \\
& \mathrm{C}_{\text {downwind }}=3.17 \times 10^{4}(\mathrm{x} / \mathrm{Q})(\mathrm{Q}) \\
& \mathrm{D}=\text { dose factor, } \mathrm{mrem} / \mathrm{pCi} \text {, } \\
& \text { for }{ }^{3} \mathrm{H}, \mathrm{D}=1.58 \times 10^{-7} \frac{\mathrm{mrem}}{\mathrm{pCi}}, \\
& \text { for }{ }^{41} \mathrm{Ar}, \mathrm{D}=8.84 \times 10^{-3} \frac{\mathrm{mrem}-\mathrm{m}^{3}}{\mathrm{pCi}-\mathrm{y}}, \\
& (\text { lnhalation rate is included in the dose factor) } \\
& \text { for }{ }^{15} \mathrm{O}, \mathrm{D}=\mathrm{D}\left({ }^{41} \mathrm{Ar}\right) \times \frac{1.74}{1.2}, \\
& \text { for }{ }^{13} \mathrm{~N}, \mathrm{D}=\mathrm{D}\left({ }^{41} \mathrm{Ar}\right) . \\
& \mathrm{D}_{\text {max individual }}=\mathrm{mrem} / \mathrm{y} .
\end{aligned}
$$

For population dose, the diffusion factors and population figures for an area within $80 \mathrm{~km}$ of the Laboratory were summed over all directions.

Dose $($ man-rem $)=3.17 \times 10^{4}\left[\sum_{i}^{n}(x / Q)_{i} P_{i}\right] Q U D ，$

where

$$
\begin{aligned}
& {\left[\sum_{i}^{n}(\chi / Q)_{i} P_{i}\right]=\begin{array}{l}
\text { Summation of the }(\chi / Q) \text { for region } i \\
\text { times the population in region } i \text { for all regions } n, \frac{\text { man-sec }}{m^{3}}
\end{array}} \\
& Q \quad=\text { release rate, } \mathrm{Ci} / \mathrm{y}, \\
& \mathrm{U} \quad=\text { inhalation rate, average individual (adult), } \\
& \mathrm{D} \quad=\text { dose factor, rem } / \mathrm{pCi}, \\
& \text { Dose }=\text { man-rem. }
\end{aligned}
$$




\section{Appendix D. Discharge Limits to \\ The Sanitary Sewer System \\ Of the City of Livermore}

Sections 18.63 and 18.66 of the Code of the City of Livermore (1959) state the discharge limits for Livermore's sanitary sewer system. These limits are as follows:

Section 18.63

No person shall discharge, or cause to be discharged, except for salt waste discharge from water softener units of any kind or description installed and in operation on or before January 31, 1966, which are regenerated by the owner thereof at the place of use of such units, any of the following described water or wastes to any public sewer unless the customer obtains a permit from the city in accordance with Section 18.65.

(a) Any liquor or vapor having a temperature higher than one hundred fifty degrees Fahrenheit.

(b) Any waters or wastes which contain more than two hundred ppm of fat, oil or grease that is petroleum-ether soluble.

(c) Any gasoline, benzene, naphtha, fuel oil or other inflammable or explosive liquid, solid or gas.

(d) Any garbage, except properly ground with a mechanical garbage grinder. Specifically excluded from the sewers are waste products resulting from the handling, storage, and sale of fruits and vegetables from other than retail produce establishments, or other foods not intended primarily for immediate consumption.

(e) Any ashes, cinders, sand, mud, straw, shavings, metal, glass, rags, feathers, tar, coal tar, asphalt, cement, plastics, woods, paunch manure or any other solid viscous substance capable of causing obstruction to the flow in sewers or other interferences with the proper economical operation of the sewage works.

(f) Any wastes or water with a $\mathrm{pH}$ lower than six and eight-tenths or higher than eight.

(g) Any waters or wastes containing total dissolved solid increments greater than three hundred and twenty-five Ppm, nor chloride increments greater than seventy-five ppm, increase during a single cycle use of the water supply.

(h) Any water or wastes having a B.O.D. greater than three hundred ppm (the average B.O.D. for residential users).

(i) Any waters or wastes containing more than three hundred ppm of suspended solids (the average suspended solids for residential users).

(j) Any waters intended to be used or used to dilute waste discharge to avoid violation of the above limitation (Order No. 586, paragraph 1).

\section{Section 18.66}

No person shall discharge or cause to be discharged any radioactive wastes into any public sewers, except where:

A. The waste is discharged in strict conformity with current Atomic Energy Commission recommendations for safe disposal of radioactive wastes.

B. The discharging of radioactive waste will not cause injury to personnel or damage to the sewer works. Any person discharging a radioactive waste to a public sewer in accordance with the provisions of the preceding paragraph shall submit to the council such report as the council may deem necessary.

In the event of an accidental spill of any radioactive material into the public sewer, the person responsible shall (a) immediately notify the plant superintendent, and (b) render such technical or other assistance to the department of public works within his power to prevent the sewage works from becoming contaminated with radioactivity. 


\title{
Appendix E. Sampling and Analytical Procedures for Environmental Monitoring at Lawrence Livermore National Laboratory
}

\author{
Descrited herein are sample collection and analysis procedures employed \\ in environmental monitoring at the Lawrence Livermore National Laboratory \\ (LI:NL). An integral part of the quality assurance program developed for \\ environmental monitoring at Livermore, these procedures provide a basis for \\ verifying that sampling and analytical activities are being performed as \\ specified. \\ The first report in this appendix describes the sampling procedures, \\ analytical determinations, and preparation for radio-counting by the LLNL \\ Hazards Control Department personnel. The second report (Manual M-122) \\ describes the methods used by the LLNL Nuclear chemistry Division.
}


Introduction

Air Sampling

Collection

Identification

Replication

Soil Sampling

collection

Identification

Replication

Water Sampling

Collection

Identification

Replication

Sewage Sampling

collection

Identification

Replication

Vegetation Sampling

collection

Identificatior.

Replication

Milk Sampling

Air Analysis

Gross Alpha and Beta counting

Beryllium

Water Analysis

Gross Alpha and Beta Activity . . . . . . . . . . . . . E-9

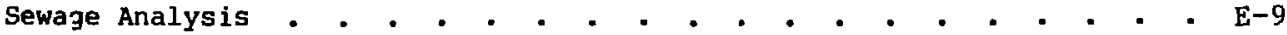

Gross Alpha and Beta Radioactivity . . . . . . . . . . . E-10

Tritium

Heavy Metals 
Written procedures have been prepared for each environmental monitoring activity at Livermore as part of the quality assurance program developed for environmental monitoring at LLNL. The sample collection and analysis procedures described herein have been reviewed and approved by those responsible for managing the monitoring program. During a program audit, such written documentation can be used to verify that sampling and analytical procedures meet program specifications.

The "Sampling" sections of this report describe the sampling procedures used for each sample medium monitored. A general description of each medium is usually provided. This is followed by the collection procedure used, provisions for sample identification, and a schedule for sample replication.

The "Analysis" sections of this report cover sample analysis. General comments on each sample medium analyzed are followed by specific descriptions of standards and calibrations, analytical procedures, and/or counting procedures as applicable for each medium.

\section{ÂIR SAMPLING}

Continuously operating air samplers are used at LLNL to measure the concentration of airborne particulate radioactivity, beryllium, and tritiated water. Samplers placed at six perimeter locations at LLNL and at nine locations at site 300 measure particulate radioactivity and beryllium. Particulate radioactivity is also measured at 10 off-site locations near the Laboratory. Tritiated-water samplers are operated at the six LLNL perimeter locations and at two off-site locations. In all cases, samplers are so placed as to provide reasonable assurance that any significant concentration of effluents from Laboratory operations would be detected regardless of local meteorology. 


\section{Particulates}

LLNL perimeter and site 300 particulate samples are collected on $5.2 \times$ $10^{-2}-m^{2}$ whatman filters, using average flow rates of 7001 iters/min. off-site samplers in the Livermore valley use $4.6 \times 10^{-3}-m^{2}$ Flanders F-700 glassfiber filters operated at a flow rate of $80 \mathrm{liters/min.} \mathrm{Particulate}$ sample flow rates are calibrated with a spirometer that has been calibrated against an immersion unit certified by the National Bureau of Standards. Flow rates of Hi-vol air samplers are verified at monthly intervals with a portable field-calibration unit.

Tritiated Water

Samples of tritiated-water vapor are collected by drawing air through silica-gel-packed columns at flow rates of about 0.51 iters/min. columns are exchanged weekly.

\section{IDENTIFICATION}

\section{Particulates}

Particulate samplers are run continuously, and sample filters are exchanged weekly. Each filter is identified by location, date on, date off, elapsed sampling time, and flow rate. Filter samples are placed in glassine envelopes and are transported to the laboratory, where this information is transferred to $\log$ sheets and each sample is given a serial number that accompanies it during analysis. Half of each LLNL perimeter and site 300 filter is used for beryllium analysis; the other half is retained for radioactivity measurements.

\section{Tritiated water}

Tritlated-water vapor samplers are run continuously, and silica-gel holders are exchanged biweekly. Each sample is identified by location, date 
on, date off, elapsed sampling time, and flow rate. This information is transferred to $\log$ sheets in the laboratory, and each sample is given a serial number that accompanies it during analysis.

\section{REPLICATION}

\section{Particulates}

At bimonthly intervals, a particulate air sampler is operated in parallel with the permanent sampler at one of the six LLNL perimeter locations. This parallel operation is continued for one month, filters are changed weekly, and both filters are submitted for analysis in the usual manner. Bimonthly rotation of the extra sampler among the six locations ensures samplers are checked annually. The replicate samples are then compared with respect to gross alpha and beta activities and to ${ }^{239} \mathrm{pu}$ content.

\section{Tritiated water}

Parallel samples of tritiated-water vapor are collected at LLNL perimeter locations during alternate months from those locations in which particulate replicates are being collected.

\section{SOIL SAMPLING}

Soil samples are collected annually within the Livermore valley and at Site 300. Radiochemical analysis of these samples serves to document any changes that may have occurred during the year. Increases in radioactivity that may result from Laboratory operations are brought to the public's attention through annual monitoring reports.

\section{COLLECTION}

Sampling sites are selected in reasonably level areas that represent undisturbec̄ soil. A $2-m$ square is marked off in each such area, and an 
8.25-cm-diam coring tool designed at LLNL is used to collect 5-cm-deep samples at the corners and center of the square.

\section{IDENTIFICATION}

The five samples of soil collected from each $2-m$ square are placed in plastic bags and are identified by number. This number is entered in a $\log$ book, together with the sampling data and a description of the samiple location. The number is used to identify the sample throughout the laboratory analysis and in the annual monitoring report.

\section{REPLICATION}

During each annual collection of soil samples, at least 108 of the sites are sampled in duplicate. In such collections, two adjacent 5-cm-deep cores are taken from the corners and center of each $2-m$ square. Separate composites of the five cores are then made, and the composites are identified by successive numbers.

\section{WATER SAMPLING}

At quarterly intervals, water samples from various sources in the Livermore Valley and at site 300 are analyzed for gross alpha and beta radioactivity and for tritium content. These analyses are performed to determine if detectable changes in radioactivity have occurred.

\section{COLLECTION}

Grab samples of such surface sources as ponds, creeks, and reservoirs are obtained with a tethered plastic pail. The samples are transferred to 4-liter plastic containers to which $6.5 \mathrm{ml}$ of concentrated $\mathrm{HCl}$ has been added. For tritium analyses, a l-1iter sample is collected in an argon-flushed glass container fitted with a ground-glass stopper. 


\section{IDENTIFICATION}

As samples are collected in the field, they are tagged with sampling location and date. In the laboratory, each sample is assigned the number that accompanies it during analysis.

REPLICATION

During each sample collection period, at least 108 of the water samples are duplicates.

\section{SEWAGE SAMPLING}

Sewage from the Laboratory is discharged into the City of Iivermore's sanitary sewer system. Radioactive or otherwise hazardous liquid wastes are first treated to reduce concentration levels to within applicable standards before they are released into the sanitary sewer. Sewage samples are collected from LLNL effluent and from the treated effluent of the Livermore Water Reclamation Plant (LWRP).

\section{COLLECTION}

\section{LLNL Effluent}

A 24-hr composite of LLNL sewage effluent is collected daily by a motorized proportional sampler. Aliquots of this composite are transported to the laboratory in 500-ml wide-mouthed polyethylene bottles.

\section{LWRP Effluent}

Daily composites of treated effluent from the LWRP are also collected by a proportional sampler. Aliquots of these composites are placed in 500-ml polyethylene bottles and are retained for weekly collection by LLNL. 
As each sewage sample is collected in the field, it is tagged with the sampling location and date of sampling. In the laboratory, each sample is assigned a number that accompanies it during analysis.

\section{REPLICATION}

\section{LLNL Effluent}

Every Wednesday, one replicate sample of LLNL effluent is taken for gross alpha, beta, and tritium. For one month each year, duplicate samples of LLNL effluent are taken each day to permit preparation of duplicate monthly composites.

\section{LWRP Effluent}

One replicate sample of LWRP effluent is taken every Wednesday and is analyzed for gross alpha, beta, and tritium. For one month each year; duplicate samples are taken each day to permit preparation of duplicate monthly composites.

\section{VEGETATION SAMPLING}

At quarterly intervals, samples of vegetation (usually native grasses) are collected throughout the Livermore valley, at site 300 , and at the off-site locations near site 300. After these samples are freeze-dried, the tritium content of the recovered water is determined with liquid-scintillation counting.

\section{COLLECTION}

Samples of vegetation are routinely collected from the same locations each sampling period. Grass samples are pulled up or cut close to the ground, and any soil is discarded. The bulk volume of these samples is usually between 
0.5 and 1 liter. As each sample is collected, it is placed in a plastic bag and frozen in a dry-ice chest to minimize water loss on the bag surface. The duplicate samples collected at each location are later transferred to a freezer in the laboratory.

IDENTIFICATION

Samples of vegetation collected in the field are tagged according to sample type and location. In the laboratory, each sample is assigned a number that accompanies it until the measurement is reported. Measurement data are permanently retained on computer cards.

\section{REPLICATION}

Two samples of vegetation are collected at each location in case the first sample is lost during analysis or the measurement is in question. At least one set of each group of samples collected is analyzed in duplicate to furnish replicate measurement data.

\section{MILK SAMPLING}

Milk samples are obtained at monthly intervals from two goat farms located about $5 \mathrm{~km}$ south and $5 \mathrm{~km}$ southeast of the Laboratory. A 3-liter portion of each milk sample is vacuum-distilled, and the tritium content of the distilled water is determined by liquid scintillation. Each 3-liter sample is then concentrated by evaporation and gamma-counted.

\section{AIR ANALYSIS}

Environmental air samples are analyzed for gross alpha and beta activity and for various gamma-emitting radionuclides, plutonium isotopes, uranium isotopes, and beryllium. 
After a four-day delay to allow for decay of the radon-thoron daughters, all environmental-radioactivity air filters are counted for alpha and beta activity. Counting is done in a Sharp gas-proportional counter using 20-min counting periods. Normally, this counter is used only for environmental samples since a duplicate counting system is provided for routine samples.

\section{Standards and Calibration}

Separate ${ }^{239} \mathrm{Pu}$ and ${ }^{90} \mathrm{Sr}$ standards on 5 -cm-diam stainless steel convoluted planchets are used for determining alpha and beta counting efficiencies, respectively. These standards are traceable to NBS or to equivalent certified sources. Counting-efficiency measurements are made with each set of filters counted, and a background count is taken at the beginning of each run and between each set of 10 samples. Records are kept of background and counting-efficiency variations in the sharp counter.

\section{BERYLLIUM}

Every month, half of each weekly LLNL perimeter and site 300 paper air filter is composited by sampling location. Two paper filters at a time are wet-ashed with a mixture of 808 nitric and 208 perchloric acid. The solutions are then evaporated to a few $\mathrm{ml}$, taking care not to allow samples to bake dry. After the samples are diluted with water, they are filtered, any residues are discarded, and the filtrates are diluted to $25 \mathrm{ml}$ with deionized water.

Any fine particulate matter passing through the filter is removed by centrifugation. The analysis is performed in an atomic emission spectrometer with a plasma source. Blanks and known spikes are subjected to the same procedure for quality control.

\section{Standards and Calibration}

Beryllium standards are prepared with 0.5 and $1 \mu \mathrm{g}$ of beryllium per $25 \mathrm{ml}$ of solution. The recorder readout of the Model 306 Perkin Elmer atomic 
absorption spectrophotometer is set at $30 \times$ absorbance expansion, and the spectrophotometer is zeroed while aspirating deionized water into the flame. Instrument responses from the $0.5-$ and $1-\mu g$ beryllium standards are then recorded. Following these calculations, the response of each monthly composite is recorded and its beryllium content is calculated from the calibration data.

WATER ANALYSIS

Environmental water samples are analyzed for gross alpha and beta activity and for tritium content.

GROSS ALPHA AND BETA ACTIVITY

A 100-ml sample or less, depending on the solid's content, is digested with a mixture of $808 \mathrm{HNO}_{3}$ and $208 \mathrm{HClO}_{4}$, and taken to dryness. The residue is taken up with $\mathrm{HNO}_{3}$ and a few milliliters of $\mathrm{H}_{2} \mathrm{O}_{2}$ (58). The solution is evaporated to about $5 \mathrm{ml}$ and transferred to a 5 -cm-diam serrated stainless steel planchet. After drying and flaming the planchet, the sample is counted for $30 \mathrm{~min}$ in a sharp 3-1/4-in proportional counter.

\section{Standards and Calibration}

The ${ }^{239} \mathrm{Pu}$ and ${ }^{90} \mathrm{Si}$ standards are used with every sample set (see the "Soil Analysis" section on standards and calibration). Instrument background measurements are made between every 10 sample-counting measurements.

\section{SEWAGE ANALYSIS}

Except for tritium analyses, ill sewage samples are wet-digested with a mixture of $208 \mathrm{HClO}_{4}$ and $808 \mathrm{HNO}_{3}$. Those sewage samples for tritium analysis are distilled to reduce self-absorption before counting. 
About $25 \mathrm{ml}$ of an $80 / 20$ mixture of $\mathrm{HNO}_{3}$ and $\mathrm{HClO}_{4}$ is added to a 500-ml aliquot ( $100 \mathrm{ml}$ of LWRP effluent), and the mixture is heated to dryness on a hotplate. The residue is taken up in $10 \mathrm{ml} \mathrm{HNO}_{3}$ and $5 \mathrm{ml} \mathrm{H}_{2} \mathrm{O}_{2}(58)$. The volume is reduced to 3-5 $\mathrm{ml}$ or a hotplate and transferred to 5-cm-diam planchets and counted.

TRITIUM

A 5-ml aliquot of sewage sample is added to $15 \mathrm{ml}$ of packard Insta-Gel in a 25-ml screw-cap polyethylene bottle. Then, $5 \mathrm{ml}$ of distilled water is used to prepare a background for the sample, and both are placed in the refrigerated detection chamber of the packard scintillation counter for a 1-hr temperature-equilibration period prior to counting. The sample and background are each counted for $100 \mathrm{~min}$.

VY METALS

Monthly composites of LLNL sewage effluent are analyzed for cadmium, chromium, copper, iron, lead, nickel, and silver.

A composite is prepared by combining daily samples of $500 \mathrm{ml}$, as follows: nitric acid (808) and $\mathrm{HClO}_{4}(208)$ are added to the first 500-ml daily sample of the month, and the mixture is reduced in volume to about 100 ml. Each subsequent day the daily sample is added and the total volume reduced to about $100 \mathrm{ml}$. After the last $500 \mathrm{ml}$ from the last day is added, the combined volume is reduced to about $50 \mathrm{ml}$. When cool, about $50 \mathrm{ml}$ deionized water is added and the liquid is filtered through No. 40 Whatman paper. The paper is then placed in a platinum crucible and digested with about $5 \mathrm{ml} \mathrm{HF}, 16 \mathrm{ml} \mathrm{HNO}_{3}$, and $4 \mathrm{ml} \mathrm{HClO}$ to a residue of about 4-5 $\mathrm{ml}$. Water is added and the liquid is refiltered. The residue is discarded. The filtrates are combined, transferred to a 100-ml volumetric flask, and brought to volume with deionized water. The metals are determined with a Perkin-Elmer Model 306 atomic absorption spectrophotometer. The required standards are prepared by dilution of 1000-ppm NBS-traceable stock solution. 
RADIOCHEMICAL METHODS OF THE SITE ENVIRONMENTAL MONITORING PROGRAM

Dianne E. Reed

A. Lee Gazlay

Alice Conover

Jeanne Marie Bazan

Michael R. Ruggieri

David W. Hosmer

Robert W. Buddemeier

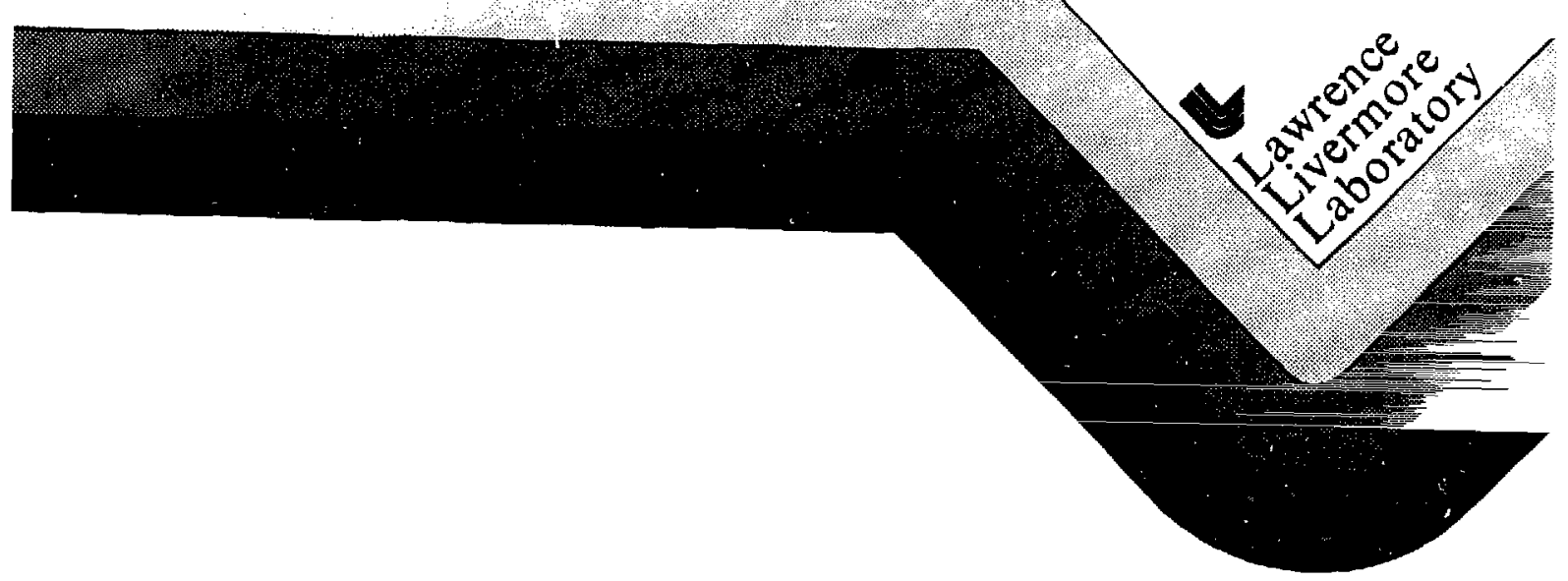


I. Overview of the Site Environmental Monitoring Program E-17

A. Sample Types E-17

B. Laboratory Facilities E-18

C. Counting Facilities E-19

1. Gamma Analys is E-19

2. Alpha Analys is E-2l

3. Beta Analys is E-2l

4. Mass Spectrometry Analys is E-22

5. Tritium Analysis E-22

II. Methods of Sample Preparation and Analysis E-24

A. Heavy Elements Analysis E-24

1. Tracers E-24

2. Air Filter Samples: Counting and Preliminary Chemistry E-24

3. Water Samples E-27
a. Preliminary Chemistry E-27
b. Plutonium Separation E-27
c. Cesium Separation E-28

4. Soil Samples E-29

a. Preliminary Treatment and Counting E-29

b. Plutonium Analys is E-30

c. Uranium Analysis $E-30$

5. Milk Analysis $t-31$

6. Anion Exchange and Heavy Element Purification Procedures E-31

a. Resin Preconditioning $t-33$

b. Column Loading $t-33$

c. Anion Exchange Separation $t-34$

d. Plutonium Purification and Plating $E-35$

e. Cesium Purification and Mounting E-38

f. Uranium Purification E-42 
B. Tritium Analys is

1. Air Moisture

a. Reservoir Preparation

$E-43$

b. Air Moisture Sample Processing

$E-45$

2. Water Samples

$E-47$

a. Distillation

$\mathrm{E}-47$

b. Enrichment

$E-48$

3. Vegetation

$E-52$

4. Goat Milk

$E-53$

III. Quality Assurance Procedures

$E-55$

A. Purpose

$E-55$

B. Storage of Sample Data and Results

$E-55$

1. Heavy Elements Analyses

$E-55$

a. Sample Sheets

$E-55$

b. Gamma Data

$E-55$

c. Alpha Data

$E-56$

d. Beta Datr

$E-56$

e. Mass Spectrometry Data

$E-56$

2. Tritium Analysis

E-56

a. Sample Sheets

$E-56$

b. Storage of Results

$E-57$

C. Sample Storage

$E-57$

1. Heavy Elements

$E-57$

2. Tritium

$E-58$

a. Vegetation and Silica Gel Samples

$E-58$

b. Milk Samples

c. Water Samples

$E-58$

d. Wine and Honey Samples

$E-58$

$E-59$

D. "QA" Samples

$t-59$

1. EML Samples

$\mathrm{t}-59$

a. EML Air Filters

b. EML Vegetation Samples

$t-59$

c. EML Water Samples

$t-60$

$t-60$

d. Storage of EML Results

$E-60$ 
2. EPA Samples E-6I

3. Duplication of Samples E-6l

a. Heavy Elements E-61

b. Tritium E-62

4. Internal Standards and Blanks E-62

a. Heavy Elements E-62

b. Tritium E-63

E. Verification of Results $\quad E-63$ 
1. Counting efficiency vs. energy for the compton suppression system E-20 (CSS) and the $20 \%$ system (IL)

2. Flow charts of heavy elements separation and purification E-25

3. Columns used for ion exchange $E-32$

4. The plutonium electroplating cell E-37

5. The cesium filtering apparatus E-4I

6. The silica gel reservoir E-43

7. Low-level tritium determination E-44

8. The freeze dry assembly E-46

9. The water distillation apparatus E-48

10. The enrichment cell assembly E-49

11. Enrichment cell head and receiving bulb for vacuum distillation E-5l

12. The milk distillation apparatus E-53

\section{TABLES}

1. Backgrounds for the compton suppression and $20 \%$ detector system E-20

2. Reagent volumes used in anion exchange separations E-35 


\section{ABSTRACT}

This methods manual describes the facilities and methods used by the Site Environmental Monitoring Program of LLNL's Nuclear Chemistry Division. Included in this report are descriptions of (1) the low-level environmental laboratory and counting facilities, (2) types of samples collected and analyzed, (3) chemical and counting methods used, and (4) quality assurance procedures.

"Work performed under the auspices of the U. S. Department of Energy by the Lawrence Livermore Laboratory under contract number W-7405-ENG-48." 
The purpose of this report is to describe the facilities and methods used by Lawrence Livermore National Laboratory's Nuclear Chemistry Division in support of the Laboratory's Site Environmental Monitoring Program. This will provide a single, easily updatable description of the current program and environmental radio-analytical techniques.

Part I describes the types of samples analyzed for the Site Environmental Program and the laboratory and counting facilities used for low-level radiocheriistry. Part II describes the specific methods of analysis, and Part III describes the quality assurance program.

1. OVERVIEW OF THE SITE ENVIRONMENTAL MONITORING PROGRAM

A. Sample Types

The Lawrence Livermore National Laboratory's Site Environmental Monitoring Program (SEMP) routinely analyzes five types of samples for heavy elements and/or tritium: air, water, soil, vegetation, and agricultural products. (Flow charts illustrating sample analyses for heavy elements and tritium are to be found on pages 9 and 28, respectively.)

Weekly air filter samples are collected from the laboratory perimeter, Site 300, and other locations near the laboratory 1 and composited monthly. Perimeter and Site 300 air filters undergo gamma analys is followed by analysis for $[239+240] \mathrm{Pu}, 238 \mathrm{Pu}, 235 \mathrm{U}, 238_{\mathrm{U}}$ and $137 \mathrm{Cs}$. Filters from other stations are analyzed only for 239+240pu and 238pu. Air moisture is collected from eight sites around the laboratory perimeter by pulling a constant air flow through a reservoir of silica gel desiccant. These weekly samples are processed to release the water which is subsequently analyzed for tritium.

Daily water samples are collected from the laboratory's main sewage line and also from Livermore's Liquid Waste Reprocessing Plant (LWRP). These samples are composited monthly and analyzed for $[239+240] \mathrm{Pu}, 238 \mathrm{pu}$ 
and ${ }^{137}$ Cs. A third water sample composed of weekly, one-gallon tap water aliquots is analyzed monthly for these same nuclides. Tritium analysis is done on water samples collected quarterly from Site 300 and the Livermore Valley. Samples may originate from wells, creeks, or ponds. Monthly rain water samples are analyzed when available. Tap water is analyzed on a quarterly basis.

Annual soil samples originate from Site 300 and locations selected at random throughout the Livermore Valley. Each sample is given a gamma scan, then analyzed for $[239+240] \mathrm{Pu}$ and $238 \mathrm{Pu}$. Site 300 soil samples are also analyzed for uranium.

Monthly vegetation samples originating from Site 300 and the Livermore Valley are freeze dried to extract their water for tritium analysis.

Goat milk is collected from local farms each month, concentrated by evaporation, and analyzed by gamma spectrometry; an aliquot of the same goat milk sample is vacuum distilled for subsequent tritium analysis of the extracted water. Locally harvested honey is combusted in a Parr bomb and the resulting water is analyzed for ${ }^{3} \mathrm{H}$. Wines from Livermore valley vineyards, plus controls from other areas, are processed in a Petersen furnace and analyzed for ${ }^{3} \mathrm{H}$.

\section{B. Laboratory Facilities}

A primary concern when maintaining an environmental level radiochemical facility is the elimination of contaminants. Several methods are employed to achieve this. The Heavy Elements and Tritium Laboratories are equipped with a single-pass air system and are located in a section of the building that is dedicated exclusively to low-level work. Entry is controlled, and visitors don disposable plastic shoe covers. Hood and counter surfaces are rountinely cleaned and the floor is periodically mopped with an EDTA solution. 
Because of tritium's rapid environmental exchange rate, special precautions are taken to maintain a low background in the Tritium laboratory. Luminous dial watches are excluded from the facility because they can release levels of tritium much higher than levels measured in routine environmental samples. For handling samples with very low tritium concentrations, argon atmosphere glove boxes are used. All glassware used for tritium analyses is scrupulously cleaned, dried in an oven at $105^{\circ} \mathrm{C}$, and then cooled in an argon atmosphere. Room air moisture is checked daily. Pieces of equipment such as drying ovens, ashing furnaces, and balances are maintained and reserved for low-level samples. The Heavy Elements Laboratory is provided with an acid fume scrubber where fumes evolved from perchloric and hydrofluoric acids are washed with a sodium hydoxide solution and then passed through an electrostatic precipitator before being exhausted. Finally, all chemicals used are analytical reagent grade, and all water is distilled and deionized.

\section{Counting Facilities}

1. Gamma Analysis

Samples requiring low-level gamma analysis are counted either on a $15 \%$ relative efficiency $\mathrm{Ge}(L i)$ spectrometer equipped with a compton suppression system ${ }^{2}$ or on a $20 \%$ relative efficiency $G(L i)$ spectrometer. Both spectrometers are constructed of materials that introduce minimal radioactivity in order to attain the lowest possible background (Table 1, page 4). Data are accumulated by an LSI-11 based Canberra 80 multichannel analyzer with a four-way mixer/router. Spectra are computer analyzed on a COC-7600 with GAMANAL, 3 a program designed for analysis of high-resolution gamma-ray spectra. The counting efficiencies of these systems vary as a function of the gamma-ray 
TABLE 1. Backgrounds for the compton suppression and 20\% detector system.

\begin{tabular}{|c|c|c|c|c|}
\hline \multirow[b]{2}{*}{$\begin{array}{l}\text { Energy } \\
(\mathrm{KeV}) \\
\end{array}$} & \multirow[b]{2}{*}{$\begin{array}{c}\text { Probable } \\
\text { Source } \\
\end{array}$} & \multicolumn{2}{|c|}{ Compton Supression System } & \multirow{2}{*}{$\begin{array}{l}20 \% \text { Detector } \\
\text { System } \\
\text { counts/min } \\
\end{array}$} \\
\hline & & $\begin{array}{l}\text { Supressed } \\
\text { counts/min }\end{array}$ & $\begin{array}{l}\text { Unsupressed } \\
\text { counts/min }\end{array}$ & \\
\hline 77.1 & 228 th & - & 0.027 & - \\
\hline 87.4 & $228 \mathrm{th}$ & 0.006 & - & - \\
\hline 186.1 & $226_{\mathrm{Ra}}$ & 0.005 & - & - \\
\hline 238.6 & $228_{\text {th }}$ & 0.039 & 0.031 & 0.020 \\
\hline 295.2 & $226 \mathrm{Ra}$ & 0.007 & 0.009 & 0.068 \\
\hline 351.9 & $226_{\mathrm{Ra}}$ & 0.016 & 0.017 & 0.111 \\
\hline 511.0 & Annihilation & 0.023 & 0.101 & 0.419 \\
\hline 583.1 & $228_{\mathrm{th}}$ & 0.003 & 0.013 & 0.011 \\
\hline 609.3 & $226 \mathrm{Ra}$ & 0.005 & 0.016 & 0.086 \\
\hline 661.6 & $137 \mathrm{Cs}$ & 0.005 & 0.004 & - \\
\hline 727.3 & $228_{\text {th }}$ & - & 0.003 & - \\
\hline 860.5 & $228_{\mathrm{th}}$ & - & 0.002 & - \\
\hline 911.1 & $228 \mathrm{Ac}$ & 0.003 & 0.006 & 0.008 \\
\hline 968.9 & $228 \mathrm{AC}$ & 0.003 & 0.004 & - \\
\hline 1119.5 & $228 A C$ & 0.002 & 0.005 & 0.017 \\
\hline 1460.8 & $40_{K}^{76}$ & 0.041 & 0.045 & 0.045 \\
\hline 1764.5 & $226 \mathrm{Ra}$ & 0.005 & 0.005 & 0.015 \\
\hline
\end{tabular}

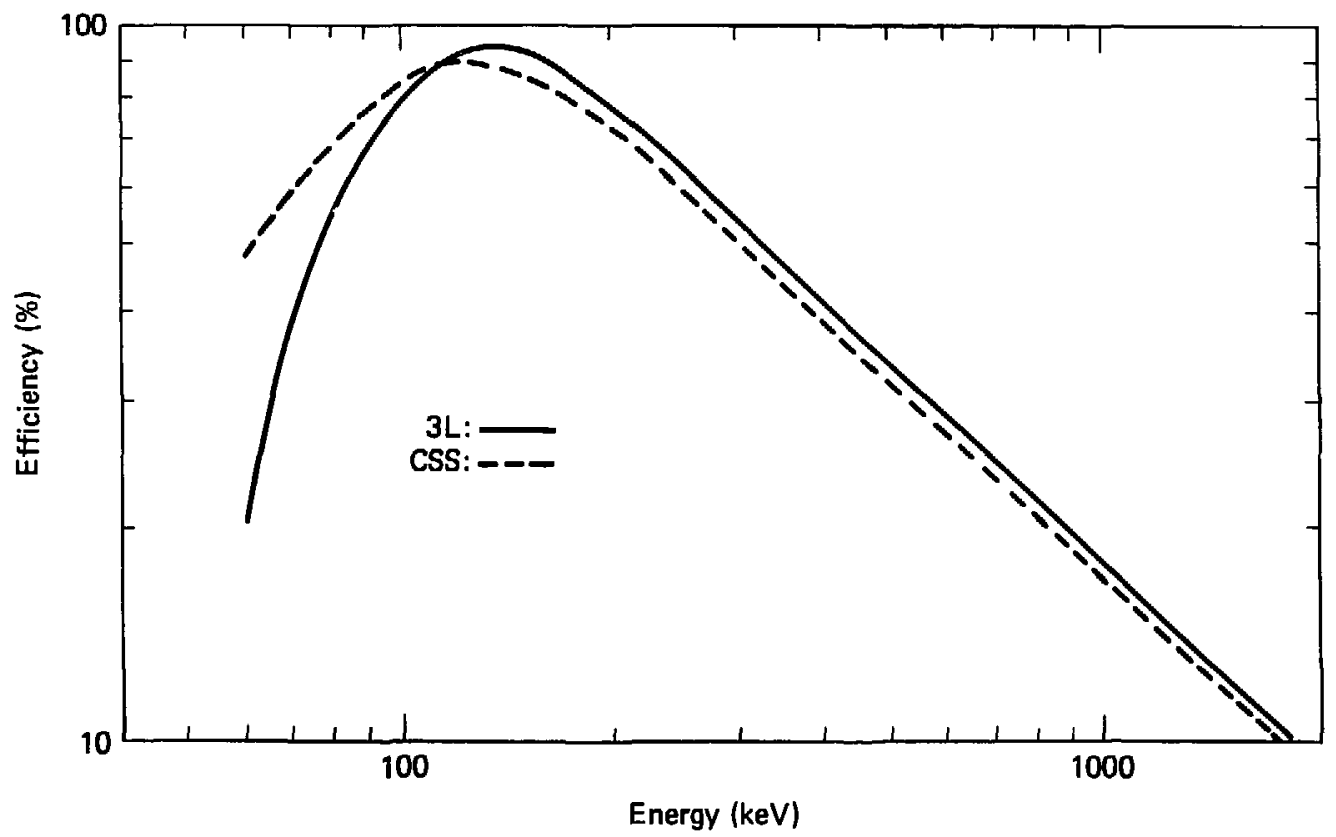

Figure 1. Counting efficiency vs energy for the compton suppression system (CSS) and the $20 \%$ system (3L). 
energies as can be seen in Fig. 1. Typical detection limits for nuclides can be determined from the information given in Fig. 1 and Table 1.

2. Alpha Analysis

Plutonium in environmental samples is determined by alpha spectrometry. Twelve $S i(L i)$ detectors with either a $450-\mathrm{mm}^{2}$ area $(20 \%$ efficient) or a $600-\mathrm{mm}^{2}$ area (26\% efficient) are used with three Nuclear Data (Model 130) 512-channel analyzers. Spectra are dumped to paper tape and computer analyzed on an LSI-11 system by ALPHAQ, a program designed for analysis of plutonium alpha spectra. These alpha detectors are reserved for low-level environmental samples in order to maintain a background of less than $1 \times 10^{-3}$ counts/min. Samples are normally counted for about $5000 \mathrm{~min}$. to produce a detection limit of about $4 \times 10^{-3} \mathrm{pCi}$ per sample.

\section{Beta Analysis}

Beta counting of ${ }^{137} \mathrm{Cs}$ is performed on $\mathrm{CsPtCl}_{6}$ precipitates deposited in a 1.8- $\mathrm{cm}^{2}$ circular area on $2.5-\mathrm{cm}$-diameter glass fiber filter paper discs. Counting efficiencies decrease from about $31 \%$ to about $21 \%$ as the sample weight increases from 1 to $50 \mathrm{mg}$. The counting system, a low-background proportional counter with an Amperex Cosmic Ray Counter as anticoincidence shield, 4 is equipped with an automated sample changer. Each sample is counted for 14 90-min. intervals. Data are analyzed on a CDC- 7600 computer by the beta reduction program BRUNHILDE. 5 
4. Mass Spectrometry Analys is

Uranium is analyzed on a sequential double-focusing 600-sector mass spectrometer with a $34-\mathrm{cm}$ radius. The instrument is supported by a PDP-8 computer that abstracts information from a multi-summing scaler, makes all corrections and calculations, and outputs the isotope ratios. This system is able to assess quantitatively the uranium content and isotopic composition of a sample down to $10 \mathrm{ng}$ of total uranium.

\section{Tritium Analysis}

Routine tritium samples are counted by liquid scintillation spectrometry. Low-level samples are electrolytically enriched ${ }^{6}$ prior to counting, and a single $5-\mathrm{ml}$ aliquot of the enriched water is analyzed. For unenriched samples, either two 7-ml or three 5-ml aliquots are used. Each counting set includes a total of eight standards (diluted to 5,6 , and $7 \mathrm{ml}$ ) to establish quench corrections, and eight blanks for background determination. Sample, standard, and background aliquots are pipetted into low-potassium liquid scintillation vials. Under controlled (red/yellow) 1ight, $10 \mathrm{ml}$ of Packard Instagei scintillation cocktail are added to each vial and mixed thoroughly. The set is loaded into the spectrometer with blanks and standards interspersed among the samples and is allowed to reach temperature equilibrium. The set is then counted for three cycles of 50 min. each per vial. Data from the spectrometer are analyzed on a CDC-7600 computer by the program SCNTCT. 7

For low-volume or low-activity samples, or where rapid ${ }^{3} \mathrm{H}$ analysis is required, a gas proportional counter is used. 8 Water samples are converted to hydrogen gas by reaction with hot magnesium turnings 
under vacuum, and the hydrogen is collected in a charcoal trap at liquid nitrogen temperature. When the sample has been warmed to room temperature, it may be transferred into the proportional counter. 9 Samples counting at less than 3 counts/min. are counted overnight, 3-8 counts/min. samples are counted all day, and samples with more than 8 counts/min. are counted only long enough to accumulate 1000 counts. The efficiency of the system is updated at least once a month with a tritium standard, and the background ( $\leq 0.7$ counts/min.) is measured over weekends. Sample results are calculated on the CDC7600 computer with the program GASCT; the file GASLIB contains standard and background information used in the calculation and is updated periodically. Routine detection limits are approximately 2 TU $\left(6.5 \times 10^{-3} \mathrm{pCi} / 1\right)$ enriched and $50 \mathrm{TU}\left(1.6 \times 10^{-1} \mathrm{pCi} / 1\right)$ unenriched for liquid scintillation counting, and 0.2 TU $\left(6.5 \times 10^{-4} \mathrm{pCi} / 1\right)$ enriched and 5 TU $\left(1.6 \times 10^{-2} \mathrm{p} f i / 1\right)$ unenriched for gas proportional counting. 
II. METHODS OF SAMPLE PREPARATION AND ANALYSIS

A. Heavy Elements Analysis

A flow chart illustrating procedures used in heavy elements analys is is shown in Fig. 2 .

1. Tracers

As a convenience, tracers and carriers used in plutonium, uranium, and cesium analyses are combined into a single mixed tracer. The tracer is diluted with $2 \mathrm{M} \mathrm{HNO}_{3}$ to provide the following in a standard 10-ml al iquot:
a) $1.3 \mathrm{pCi} 242 \mathrm{pu}$
b) $4.36 \times 10^{13}$ atoms $233 \mathrm{U}$
c) $25 \mathrm{mg} \mathrm{CsCl}$

2. Air Filter Samples: Counting and Preliminary Chemistry

1) Composite the month's air filters from the laboratory perimeter stations and seal in a $216-\mathrm{cm}^{3}$ aluminuni can for gamma analysis. Upon compleition of a 1500-min. count, separate the air filters, by station, into platinum crucibles. Care should be taken to mininize cross-contamination.

2) Composite the month's air filters from the site 300 stations and seal in a $216-\mathrm{cm}^{3}$ aluminum can for gamma analysis. Upon completion of a 1500-min. count, place all of the Site 300 air filters into a single Pyrex beaker.

3) Place other non-gamma counted Livermore Valley air filters into individual Pyrex beakers by station.

4) Add $10 \mathrm{ml}$ of mixed tracer to each sample.

5) Dry the air filters in a drying oven at $110^{\circ} \mathrm{C}$ for at least four hours.

6) Ash the air filters at $475^{\circ} \mathrm{C}$ for at least two days. 


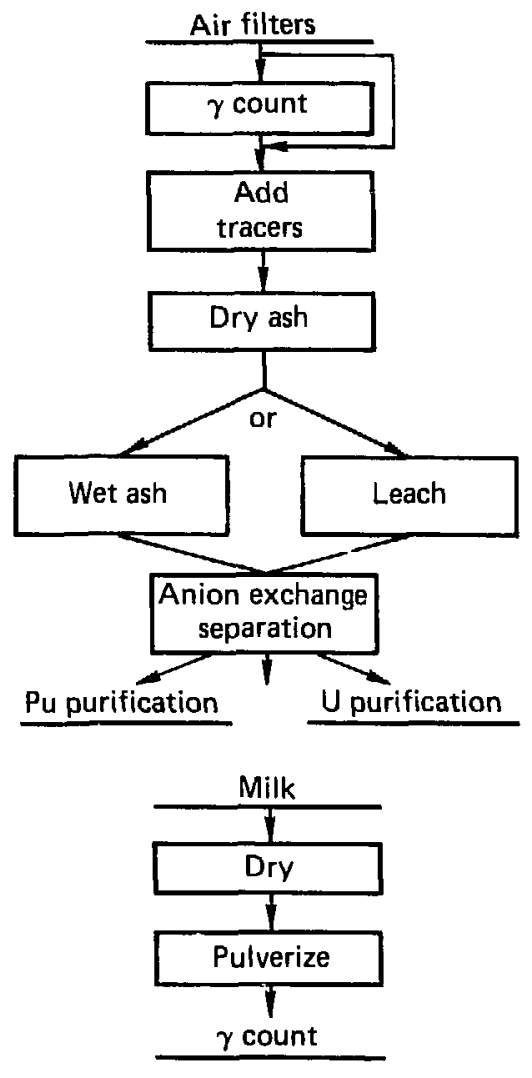

\begin{tabular}{l} 
Cs purification \\
\hline AMP extraction \\
\hline $\begin{array}{c}\text { Cation exchange } \\
\text { purification }\end{array}$ \\
\hline $\begin{array}{c}\text { Precipitate } \\
\mathrm{Cs}_{2} \mathrm{PtCl}_{6}\end{array}$ \\
\hline Filter/Mount \\
\hline $\mathrm{B}^{-}$count \\
\hline
\end{tabular}
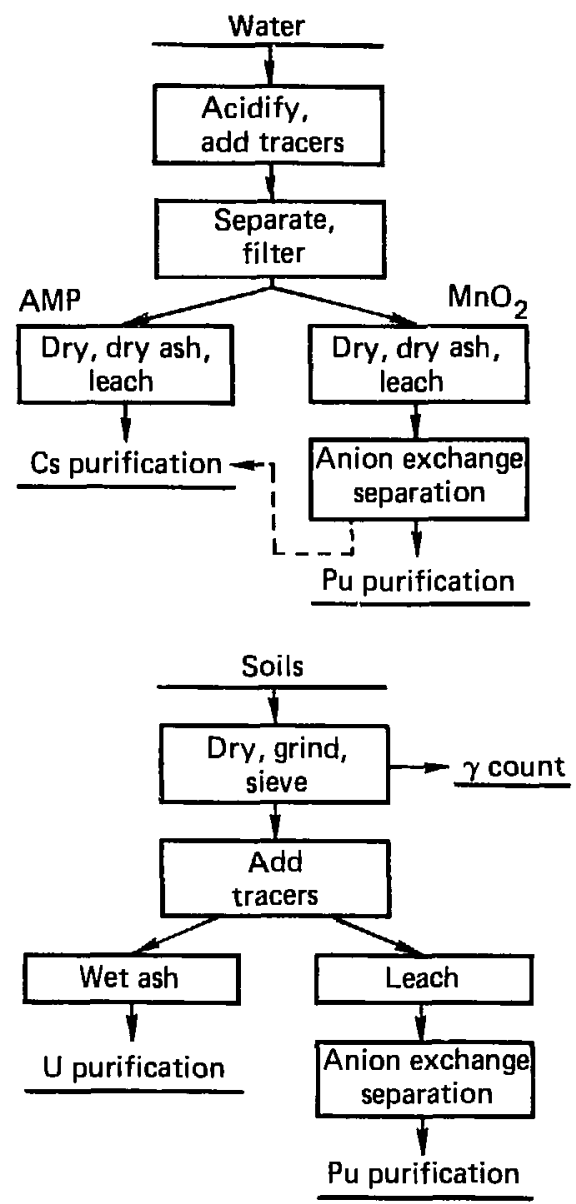

$\frac{\text { Pu purification }}{1}$

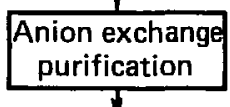

U purificátion

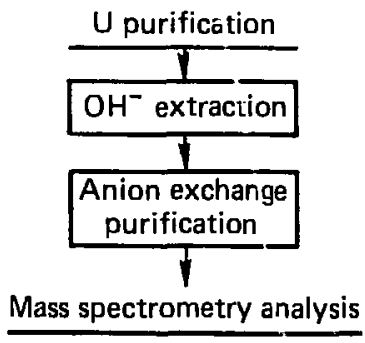

Figure 2. Flow charts of the heavy elements separation and purification procedures. 
7a) For samples that require uranium analys is (LLNL perimeter, Site 300, standards, and blanks):

i) If the ashed samples are not already in platinum beakers, transfer them from Pyrex to platinum beakers with minimum conc nitric acid $\left(\mathrm{HNO}_{3}\right)$.

ii) Add conc $\mathrm{HNO}_{3}$ to a total volume of $30 \mathrm{ml}$.

iii) Add $30 \mathrm{ml}$ of conc hydrofluoric acid (HF).

iv) Heat the sample to dryness in the acid fume scrubber.

v) Add $30 \mathrm{ml}$ each of conc $\mathrm{HNO}_{3}$ and conc perchloric acid $\left(\mathrm{HClO}_{4}\right)$.

vi) Heat until fuming ceases, in the scrubber.

vii) Dissolve as much of the residue as possible by heating with $20 \mathrm{ml}$ of $8 \mathrm{M} \mathrm{HNO}_{3}$ and a few drops of $30 \%$ hydrogen peroxide $\left(\mathrm{H}_{2} \mathrm{O}_{2}\right)$.

viii) Transfer the solution to a $250-\mathrm{m} 1$ plastic bottle using minimal 8 M $\mathrm{HNO}_{3}$.

7b) For samples that require only plutonium analysis (Livermore Va11ey):

i) Treat the ashed filter with $50 \mathrm{ml}$ of conc $\mathrm{HNO}_{3}$ and $20 \mathrm{ml}$ of conc hydrochloric acid (HCl).

ii) Evaporate the sample to a final volume of $50 \mathrm{ml}$.

iii) Transfer the sample to a $250-\mathrm{ml}$ plastic bottle using minimal $8 \underline{\mathrm{M}} \mathrm{HNO}_{3}$.

iv) Make the sample approximately $8 \mathrm{M} \mathrm{HNO}_{3}$ by doubling the volume with $0.5 \underline{M} \mathrm{HNO}_{3}$.

8) Treat al1 samples with $0.5 \mathrm{~g}$ of sodium nitrite $\left(\mathrm{NaNO}_{2}\right)$ to adjust the plutonium valence to +4 for anion exchange, and wait at least four hours before further processing. 
9) Proceed to Section IIA-6 (page 15) for anion exchange separation and heavy element purification procedures.

3. Water Samples

a. Preliminary Chemistry

1) For laboratory sewage and LWRP samples: add $5 \mathrm{ml}$ of $3 \underline{M} \mathrm{HCl}$ to each 500-ml aliquot before combining these for the monthly composite. Add $10 \mathrm{ml}$ of mixed tracer to the composite sample.

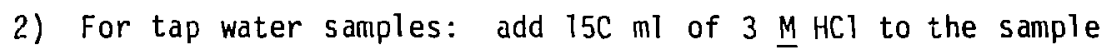
vessei followed by $10 \mathrm{ml}$ of mixed tracer. Combine the weekly tap water aliquots in the vessel with stirring.

b. Plutoniuin Separation 10

1) Add $10 \mathrm{ml}$ of saturated potassium permanganate $\left(\mathrm{KMnO}_{4}\right)$ to the sample.

2) Add $70 \mathrm{ml}$ of $10 \mathrm{M} \mathrm{NaOH}$ and check the $\mathrm{pH}$ to make sure that it is basic.

3) Coprecipitate plutonium with manganese dioxide $\left(\mathrm{MnO}_{2}\right)$ by slowly adding $150 \mathrm{ml}$ of $0.1 \mathrm{M}$ manganous chloride $\left(\mathrm{MnCl}_{2}\right)$. As $\mathrm{MnCl}_{2}$ is added, maintain a basic $\mathrm{pH}$ by addition of small amounts of $3 \underline{\mathrm{M} \mathrm{NaOH}}$.

4) Pump the sample through a $1-\mu$ cotton filter cartridge to remove the $\mathrm{MnO}_{2}$ from solution. In the laboratory sewage sample, solid material is present before separation. This matter has been found to contain a significant percentage of the ${ }^{137} \mathrm{Cs}$ in the sample. Therefore, cesium is later separated from this filter, by anion exchange (see Section IIA-6, page 15).

5) Separate the cotton filter from the filter's plastic core and place it in a 1-1 beaker. 
6) Dry the filter in an oven at $110^{\circ} \mathrm{C}$ for two days.

7) Ash the filter at $475^{\circ} \mathrm{C}$ for two days.

8) Add $100 \mathrm{ml}$ of conc $\mathrm{HNO}_{3}$ to the ashed $\mathrm{MnO}_{2}$ cartridge and heat for at least an hour.

9) Add $30 \% \mathrm{H}_{2} \mathrm{O}_{2}$ until the $\mathrm{MnO}_{2}$ has dissolved.

10) Evaporate the sample to about $50 \mathrm{ml}$. Transfer the sample to a 250-ml plastic bottle.

11) Make the sample approximately $8 \mathrm{MHNO}_{3}$ by doubling the sample vol ume with $0.5 \mathrm{M} \mathrm{HNO}_{3}$.

12) Add approximately $0.5 \mathrm{~g}$ of $\mathrm{NaNO}_{2}$ to prepare for anion exchange. Wait at least four hours before continuing processing.

13) Proceed to Section IIA-6 (page 15) for anion exchange separation and heavy element purification procedures.

c. Cesium Separation 11

1) Add $150 \mathrm{ml}$ of $3 \underline{\mathrm{M} \mathrm{HCl}}$ to the plutonium separation filtrate (from step 4 in plutonium separation above) and verify that the $\mathrm{pH}$ is $1-3$.

2) Add $5 \mathrm{~g}$ of AMP as a distillad $\mathrm{H}_{2} \mathrm{O}$ slurry.

3) Pump the sample through a $1-\mu$ cotton filter cartridge to separate the AMP.

4) Separate the cotton filter from the filter's plastic core.

5) Dry the filter in an oven at $110^{\circ} \mathrm{C}$ for two days.

6) Ash the filter at $475^{\circ} \mathrm{C}$ for two days.

7) Add 3 M HCl to the ashed AMP cartridge to form a siurry.

8) For the laboratory sewage sample, comoine the cesium eluate from the anion exchange separation with the $\mathrm{MnO}_{2}$ cartridge fraction. 
9) Heat the slurry and dissolve the AMP with $10 \mathrm{M} \mathrm{NaOH}$.

10) Add a $60-\mathrm{ml}$ excess of $10 \mathrm{M} \mathrm{NaOH}$ to the sample.

11) Evaporate the sample to a volume of $150-200 \mathrm{ml}$ under low heat $\left(<70^{\circ} \mathrm{C}\right)$.

12) Centrifuge, wash twice, combine supernates, and discard the solids in the sample.

13) Acidify the supernate to $\mathrm{pH} 1-3$ with $3 \underline{\mathrm{M}} \mathrm{HCl}$. NOTE: If a white silicate precipitate appears, adjust the $\mathrm{pH}$ to 5-8 with $1 \mathrm{M} \mathrm{NaOH}$, centrifuge to remove the precipitate, and repeat step 13.

14) Dilute the sample to $600 \mathrm{ml}$ with $\mathrm{H}_{2} \mathrm{O}$.

15) Add 1 gram of AMP as a slurry and stir.

16) Al low the AMP to settle overnight.

17) Proceed to Section IIA-6e (page 22) for cesium purification and mounting procedures.

\section{Soil Sampies}

a. Preliminary Treatment and Counting

1) Dry the soil sample at $110^{\circ} \mathrm{C}$ for at least two days.

2) Remove rocks and pebbles with diameters greater than $0.5 \mathrm{~cm}$.

3) Pulverize the remaining material in a grinding mill.

4) Pass the sample through a 32-mesh sieve. Discard the fraction that is greater than 32 mesh.

5) Thoroughly blend the sample.

6) Pack a 216- $\mathrm{cm}^{3}$ tared aluminum can with the sieved soil for gamma analysis. 
b. Plutonium Analysis

(Acid leach method 12 )

1) Weigh a 100-g aliquot of the prepared soil.

2) Add $10 \mathrm{ml}$ of the mixed tracer.

3) Leach the sample by heating for four hours with $300 \mathrm{ml}$ of conc $\mathrm{HNO}_{3}$ and $100 \mathrm{ml}$ of conc $\mathrm{HCl}$. Control foaming with a few drops of n-octanol.

4) Vacuum filter the hot sample through a buchner funnel fitted with 934 AH glass fiber filter paper. The supernate should be clear. Wash the sample twice with approximately $50 \mathrm{ml}$ hot $8 \underline{\mathrm{M}}$ $\mathrm{HNO}_{3}$ each wash.

5) Determine the molarity of the supernate by titrating a $0.5-\mathrm{ml}$ aliquot with standardized $1 \mathrm{M} \mathrm{NaOH}$ to a phenolphthalein end point.

6) Adjust the molarity of the solution to $8 \mathrm{M} \mathrm{HNO}_{3}$ with appropriate volume of 0.5 M $\mathrm{HNO}_{3}$.

7) Add about 1 gram of $\mathrm{NaNO}_{2}$ to the sample to prepare for anion exchange. Wait at least four hours before continuing processing.

8) Proceed to Section IIA-6 (page 15) for anion exchange separation and Pu purification procedures.

c. Uranium Analysis

1) Weigh 1 gram of prepared soil into a platinum beaker.

2) Add $10 \mathrm{ml}$ of the mixed tracer.

3) Add $10 \mathrm{ml}$ of conc $\mathrm{HNO}_{3}$.

4) Add $10 \mathrm{ml}$ of conc HF.

5) Heat the sample to near dryness.

6) Reconstitute the sample with an additional $10 \mathrm{ml}$ of conc HF. 
7) Evaporate the sample to dryness.

8) Treat the residue with $20 \mathrm{ml}$ of conc $\mathrm{HNO}_{3}$.

9) Add $20 \mathrm{ml}$ of conc $\mathrm{HC}_{4} 10_{4}$.

10) Heat the sample until the white fumes of $\mathrm{HClO}_{4}$ are no longer evolved.

11) Dissolve the residue with a minimum volulme of $8 \underline{\mathrm{M}} \mathrm{HNO}_{3}$ and 2-4 $\mathrm{ml}$ of $30 \% \mathrm{H}_{2} \mathrm{O}_{2}$ and heat.

12) Transfer the sample to a $40 \mathrm{ml}$ Pyrex centrifuge tube with minimum $\mathrm{H}_{2} \mathrm{O}$.

13) Proceed to Section IIA-6d (page 19) for the U purification procedure.

5. Milk Analysis

1) Pour 3 liters of milk into a tared 4-liter beaker.

2) Evaporate the milk sample slowly, with continuous stirring, to approximately 0.5 liter.

3) Dry the milk for two days at $110^{\circ} \mathrm{C}$.

4) Weigh the dried milk solids.

5) Pulverize the milk solids in a blender.

6) Fill a tared $216-\mathrm{cm}^{3}$ aluminum can with the pulverized sample.

7) Weigh the can for gamma analysis, and gamma count.

6. Anion Exchange Separation and Heavy Element Purification Procedures

It is evident from flow charts of the various purification procedures (Fig. 2, page 9) that, excluding the milk chemistry, each purification involves an ion exchange separation. Four different column sizes are used for ion exchange (Fig. 3, page 16). The techniques of column preparation and ion exchange separations are similar for all procedures, differing only in the size of the columns used and in the amounts of necessary reagents. 

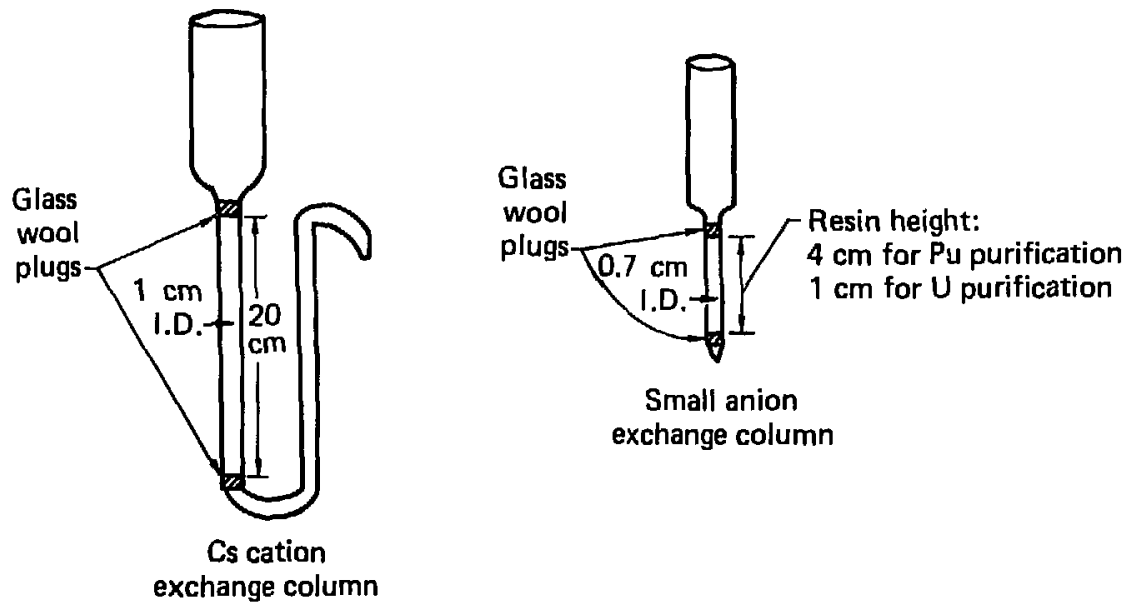

Small anion

exchange column

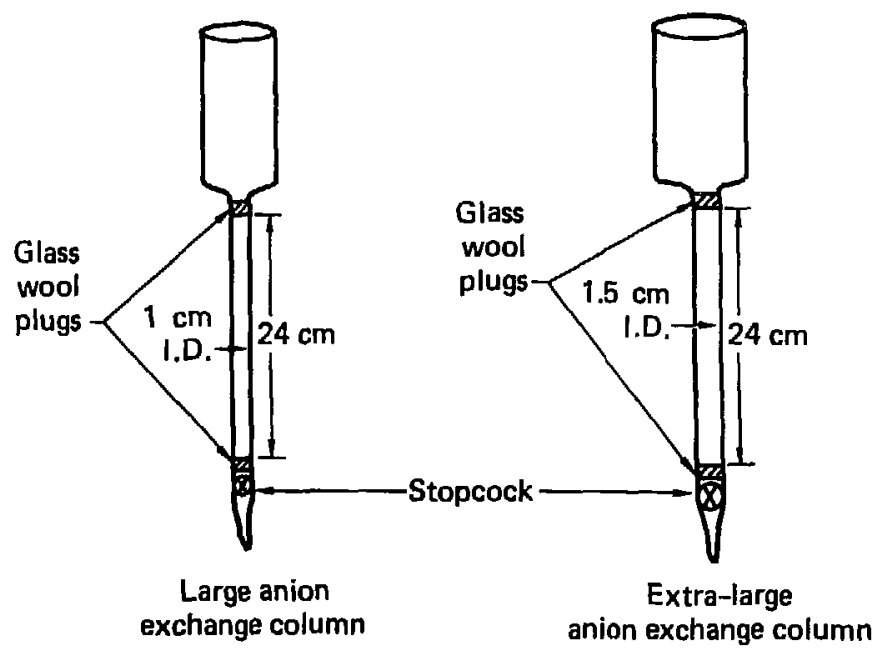

Figure 3. Columns used for ion exchange. 


\section{a. Resin Preconditioning}

1) Precondition $1-\times 8,50-100$ mesh $\left(\mathrm{Cl}^{-}\right.$form) and $\mathrm{AGl}-\mathrm{X8}, 100-200$ mesh anion exchange resins by putting the resin in a 2-1 buchner funnel fitted into a 4-1 filter flask, and
a) washing with $8 \mathrm{MHNO}_{3}$ (2-1 acid per liter resin).
b) washing with conc $\mathrm{HCl}$ (2-1 acid per liter resin).
c) Repeatedly washing with $\mathrm{H}_{2} \mathrm{O}$ until the eluate is of neutral pH.

2) Prepare anion exchange resin for uranium purification as follows ${ }^{13}$ :

a) Grade AG 1-X8, 100-200 mesh resin in a counterflow fluid grader, collecting the $30-70 \mathrm{~mm} / \mathrm{min}$ fraction.

b) Wash about $250 \mathrm{ml}$ of the resin with $2 \mathrm{M} \mathrm{NaOH}$.

c) Rinse the resin with 8 liters of $\mathrm{H}_{2} \mathrm{O}$.

d) Convert the resin to sulfate form with $1 \mathrm{M} \mathrm{H}_{2} \mathrm{SO}_{4}$.

e) Wash the resin with $4 \mathrm{I}$ of $0.06 \mathrm{M} \mathrm{H}_{2} \mathrm{SO}_{4}$.

3) Precondition Bio-Rex 40, 20-50 mesh cation exchange resin for cesium purification with repeated $5 \% \mathrm{NaCl}$ washes until the eluate is clear.

b. Column Loading

1) Fill the column with $\mathrm{H}_{2} \mathrm{O}$.

2) Insert a glass wool plug into the bottom of the column to prevent resin 10ss. Eliminate any air bubbles from this plug.

3) Load the required amount of ion exchange resin as an $\mathrm{H}_{2} \mathrm{O}$ slurry and allow the resin to settle.

4) Insert a glass wool plug into the top of the column. Eliminate any air bubbles from the plug before pressing it onto the top of the resin. 
5) Rinse out the column with $\mathrm{H}_{2} \mathrm{O}$ to remove any loose resin.

\section{c. Anion Exchange Separation}

See Table 2 (page 19) for the approriate volumes of reagents to be used for each particular sample type.

1) Extra-large columns should be used for soil samples, while large columns should be used for water and air filter samples. Load the appropriate size column with preconditioned 1- $\times 8$, 50-100 mesh ( $\mathrm{Cl}^{-}$form) anion exchange resin to a height of about $24 \mathrm{~cm}$, followed by $1 \mathrm{~cm}$ of preconditioned AGl-X8 100-200 mesh ( $\mathrm{Cl}^{-}$form) anion exchange resin.

2) Condition the column with the appropriate volume of $8 \mathrm{M} \mathrm{HNO}_{3}$.

3) Filter the sample through 934 AH glass fiber filter paper onto the column and, if cesium analysis is desired, collect the eluate. The eluate from this step and that from the next step comprise the cesium fraction (proceed to Section IIA-6e, page 22, for cesium purification and mounting).

4) When the liquid has run through the column, rinse the column reservoir twice with $8 \underline{\mathrm{M}} \mathrm{HNO}_{3}$; then wash the resin with the appropriate volume of $8 \underline{\mathrm{M}} \mathrm{HNO}_{3}$.

5) Rinse the column reservoir with conc $\mathrm{HCl}$, then wash the resin with the appropriate volume of conc $\mathrm{HCl}$ to remove thorium contamination.

6) Elute plutonium with the appropriate volume of freshly prepared $\mathrm{NH}_{4} \mathrm{I}-\mathrm{HCl}$ solution ( $1.5 \mathrm{~g} \mathrm{NH}_{4} \mathrm{I}$ per $100 \mathrm{ml}$ conc $\mathrm{HCl}$ ). Add several $\mathrm{ml}$ conc $\mathrm{HNO}_{3}$ and evaporate to dryness. Proceed to Section IIA-6d (page 19) for plutonium purification processing. 
7) Elute uranium with the appropriate volume of $0.1 \mathrm{M} \mathrm{HCl}$ and evaporate to dryness. Proceed to Section IIA-6f (page 26) for uranium purification processing.

TABLE 2. Reagent volumes used in anion exchange separations.

\begin{tabular}{|c|c|c|c|c|c|c|}
\hline Sample type & Column size & $\begin{array}{l}8 \mathrm{MMNO} \\
\text { condition } \\
\text { step, ml } \\
\end{array}$ & $\begin{array}{l}8 \mathrm{M} \mathrm{HNO}_{3} \\
\text { wash, ml } \\
\end{array}$ & $\begin{array}{l}\text { conc } H C l \\
\text { wash, ml } \\
\end{array}$ & $\begin{array}{l}\mathrm{NH}_{4} \mathrm{I} / \\
\mathrm{HCl}, \mathrm{ml} \\
\end{array}$ & $\begin{array}{l}0.1 \mathrm{M} \\
\mathrm{HCl}, \mathrm{ml}\end{array}$ \\
\hline air filter & large & 50 & 50 & 100 & 80 & 80 \\
\hline water & large & 50 & 50 & 100 & 80 & -- \\
\hline soil & $x-1$ arge & 100 & 300 & 200 & 150 & -- \\
\hline
\end{tabular}

d. Plutonium Purification and Plating ${ }^{14}$

Purification:

1) Dissolve the residue by heating with a few milliliters of conc $\mathrm{HCl}$ and several drops of $30 \% \mathrm{H}_{2} \mathrm{O}_{2}$. Make sure that excess $\mathrm{H}_{2} \mathrm{O}_{2}$ has been evaporated before proceeding.

2) Transfer the solution to a 40-ml plastic centrifuge tube with a minimum amount of conc $\mathrm{HCl}$.

3) Add $0.5 \mathrm{~g}$ of $\mathrm{NaNO}_{2}$.

4) Cap the tube and centrifuge to remove the insoluble sodium chloride $(\mathrm{NaCl})$ formed by the $\mathrm{NaNO}_{2}$ addition.

5) Load a small ion exchange column to a height of about $4 \mathrm{~cm}$ with preconditioned Dowex 50-100 mesh anion exchange resin (Cl- form).

6) Cordition the resin with $20 \mathrm{ml}$ of conc $\mathrm{HCl}$.

7) Load the sample onto the column. Repeat the centrifuging and and loading procedures twice more.

8) When the liquid has run through the column, rinse the sides of the column reservoir with conc $\mathrm{HCl}$, then wash the resin with $20 \mathrm{ml}$ of conc $\mathrm{HCl}$. 
9) Rinse the column reservoir with $8 \underline{M} \mathrm{HNO}_{3}$ and then wash the resin with $20 \mathrm{ml}$ of 8 M NHO 3 .

10) Re-ririse the column reservoir with conc HCL and wash the resin with another $20 \mathrm{ml}$ of conc $\mathrm{HCl}$.

11) Elute the plutonium with $20 \mathrm{ml}$ of freshly prepared $\mathrm{NH}_{4} \mathrm{I}-\mathrm{HCl}$ solution ( $1.5 \mathrm{~g} \mathrm{NH}_{4} \mathrm{l}$ per $100 \mathrm{ml}$ conc $\mathrm{HCl}$ ).

12) Add several $\mathrm{ml}$ conc $\mathrm{HNO}_{3}$ to the eluate from step 12 and evaporate to dryness.

Plating:

13) Assemble the electroplating cell15 (Fig. 4, page 21).

a) Clean a polished 2.54-cm-diam. $\times 0.05-c m-t h i c k$ stainless steel disc, engraved with the sample identification number.

b) Place the disc at the bottom of a stainless steel base to serve as the cathode.

c) Place a teflon washer on top of the disc and secure a labelled glass chiminey in place with a teflun support, stainless steel ring, and brass wing nuts to form a watertight seal around disc.

d) Fill the cell with water to test for leakage.

14) Add $1 \mathrm{ml}$ conc sulfuric acid $\left(\mathrm{H}_{2} \mathrm{SO}_{4}\right)$ to the residual salts of plutonium eluate.

15) Heat the sample until copious white fumes are evolved.

16) When the sample has cooled, dilute with $2 \mathrm{ml}$ of $1 \underline{\mathrm{M}_{2} \mathrm{SO}_{4}}$.

17) Add two drops of 0.1 methyl red indicator.

18) Transfer the sample to the electroplating cell with a minimum volume of $\mathrm{I}_{\mathrm{M} \mathrm{H}} \mathrm{H}_{4}$. 


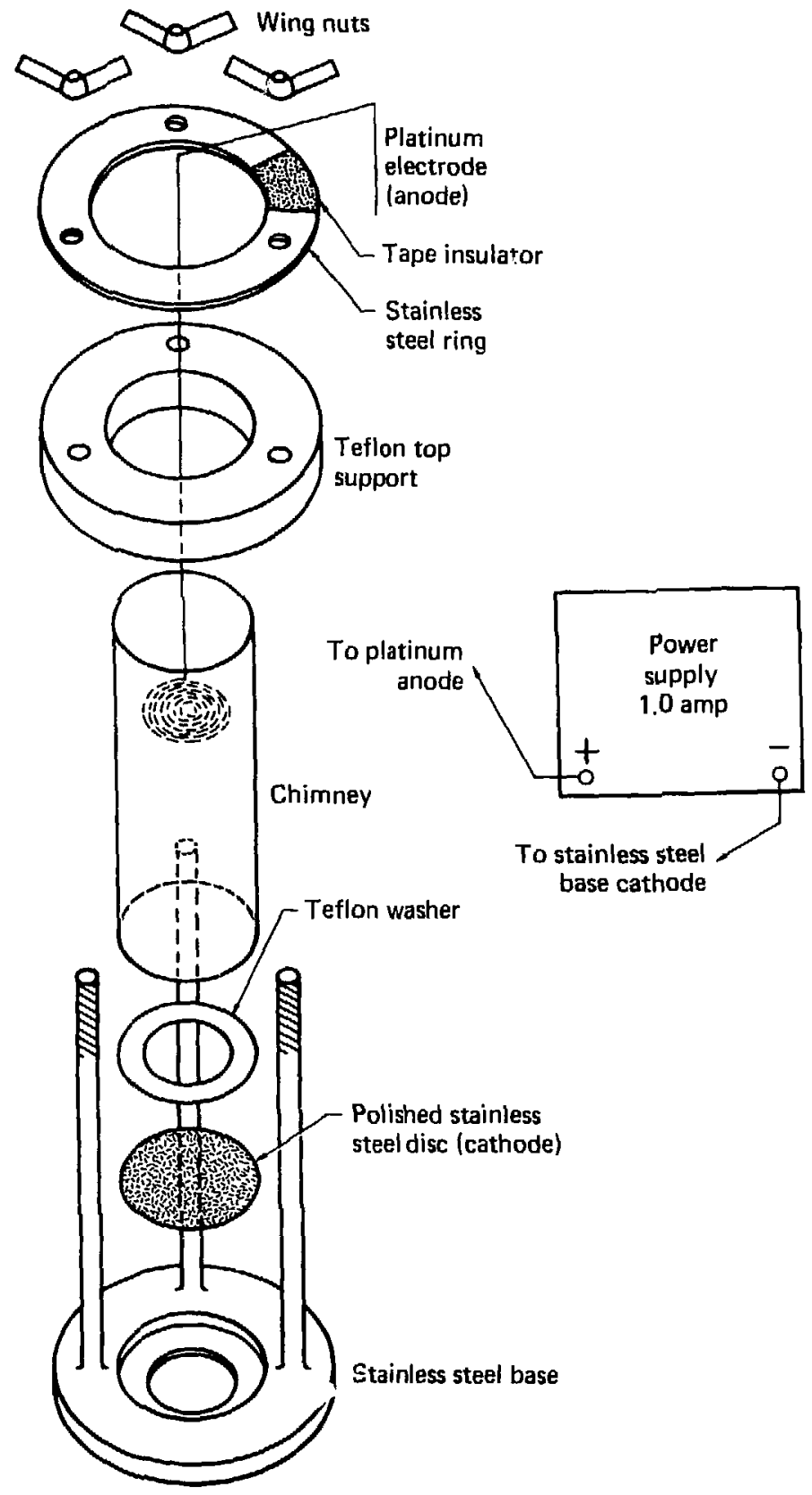

Figure 4. The plutonium electroplating cell. 
19) Titrate the sample to a yellow end point (pH 6.2) with conc $\mathrm{NH}_{4} \mathrm{OH}$.

20) Back titrate five drops past the red end point (pH 4.4) with $1 \underline{\mathrm{M} \mathrm{H}_{2} \mathrm{SO}_{4}}$.

21) Use a 0.064-cm-thick platinum wire, coiled at the bottom, as the anode.

22) Electroplate at 1.0 amp for $70-90 \mathrm{~min}$.

23) Before completion, add $1 \mathrm{ml}$ of conc $\mathrm{NH}_{4} \mathrm{OH}$ to the cell and continue plating for one min.

24) As quickly as possible:

a) disengage the power supply;

b) remove the anode;

c) discard the solution;

d) rinse the cell with $0.1 \mathrm{M} \mathrm{NH}_{4} \mathrm{OH}$;

e) disassembie the cell;

f) rinse the disc with $0.1 \mathrm{M} \mathrm{NH}_{4} \mathrm{OH}$;

g) rinse the disc with acetone.

25) Determine $[239+240] \mathrm{Pu}$ and ${ }^{238} \mathrm{Pu}$ on the disc by alpha spectrometry.

e. Cesium Purification and Mounting

Purification:

1) Add sufficient amount of $10 \mathrm{M} \mathrm{NaOH}$ to the cesium fraction from the anion exchange separation to make the solution $\mathrm{pH} 10-12$.

2) Centrifuge to remove any $\mathrm{Fe}(\mathrm{OH})_{3}$ precipitate.

3) Decant the supernate into a large beaker.

4) Dissolve the $\mathrm{Fe}(\mathrm{OH})_{3}$ in a minimum amount of $8 \underline{\mathrm{M}} \mathrm{HNO}_{3}$.

5) Dilute the solution with about $10 \mathrm{ml}$ of $\mathrm{H}_{2} \mathrm{O}$. 
6) Re-precipitate $\mathrm{Fe}(\mathrm{OH})_{3}$ with $10 \mathrm{M} \mathrm{NaOH}$.

7) Centrifuge and combine the supernates.

8) Repeat steps 4 through 7 once.

9) Acidify the supernates with $3 \underline{M} H C l$ to $\mathrm{pH} 1-3$.

10) Dilute the sample to $600 \mathrm{ml}$ with $\mathrm{H}_{2} \mathrm{O}$.

11) Add 1 gram of ammonium molybdophosphate (AMP) as a slurry with $\mathrm{H}_{2} \mathrm{O}^{12}$. Cesium in the solution will complex with the AMP.

12) Allow the sample to equilibrate and the AMP to settle overnight.

13) Decant and discard the supernate.

14) Transfer the AMP complex to a 40-ml Pyrex centrifuge tube with a minimum amount of $\mathrm{pH} 2-3 \mathrm{HCl}$.

15) Centrifuge and discard the supernate.

16) Dissolve the AMP complex with a combination of $1 \mathrm{ml} \mathrm{H}_{2} \mathrm{O}, 1 \mathrm{ml}$ of a $0.75 \underline{M ~ N a O H}$ - 2\% EDTA solution, and $1 \mathrm{ml} 10 \underline{M} \mathrm{NaOH}$.

17) Load preconditioned Bio-Rex 40, 20-50-mesh cation exchange resin into a cesium ion-exchange column to a height of approximately $20 \mathrm{~cm}$.

18) Prime the cation exchange resin with $250 \mathrm{ml}$ of $5 \% \mathrm{NaCl}$.

19) Slowly load the sample by pipet.

20) After the liquid has drained from the reservoir, rinse the column sides with three 2-ml aliquots of $\mathrm{H}_{2} \mathrm{O}$.

21) Wash the resin with $45 \mathrm{ml}$ of $\mathrm{H}_{2} \mathrm{O}$.

22) Wash the column with $200 \mathrm{ml}$ of $0.75 \mathrm{M} \mathrm{HCl}$ administered in 50-ml al iquots.

23) Elute the cesium with $125 \mathrm{ml}$ of $3.0 \mathrm{M} \mathrm{HCl}$.

24) To permit reuse of the cation exchange resin, wash the resir. with $250 \mathrm{ml}$ of $\mathrm{H}_{2} \mathrm{O}$. This resin can be reused up to five times.

25) Evaporate the cesium eluate to dryness. 
Mounting:

26) Prepare a tared filter disc:

a) Cut a 2.54-Cm-dia 934 AH glass fiber filter paper disc.

b) Wash the disc with $\mathrm{H}_{2} \mathrm{O}$, then acetone.

c) Dry the disc under infrared light.

d. Weigh the disc to $+0.1 \mathrm{mg}$.

27) Dissolve the residual salts in the cesium eluate with $2-3 \mathrm{ml}$ of 3 M HCl and heat.

28) Transfer the solution to a 40-ml plastic centrifuge tube with minimum $\mathrm{H}_{20}$.

29) Make the solution basic by adding $3 \mathrm{M} \mathrm{NaOH}$.

30) Add $4 \mathrm{ml} 0.12 \mathrm{M}$ chloroplatinic acid $\left(\mathrm{H}_{2} \mathrm{PtCl}_{6}\right)$. Cesium will be precipitated as $\mathrm{Cs}_{2} \mathrm{PtCl}_{6}$. If this does not occur instantly, place the centrifuge tube in an ultrasonic bath for about $30 \mathrm{~s}$.

31) Cool the sample in an ice bath for 30-40 min.

32) Assemble the cesium filtering apparatus (Fig. 5, page 25).

33) Filter the sample through the tared disc depositing the $\operatorname{CsPtCl}_{6}$.

34) Remove the chimney from the apparatus.

35) Rinse tha disc (under vacuum) with $2 \mathrm{ml}$ of cold $\mathrm{H}_{2} \mathrm{O}$.

36) Rinse the disc with $2 \mathrm{ml}$ of cold acetone.

37) Dry the filter under infrared light.

38) Weigh the sample.

39) Mount the disc on a plastic base with a plastic ring holding a mylar cover.

40) Determine the cesium by beta spectrometry with a weight yield correction. 


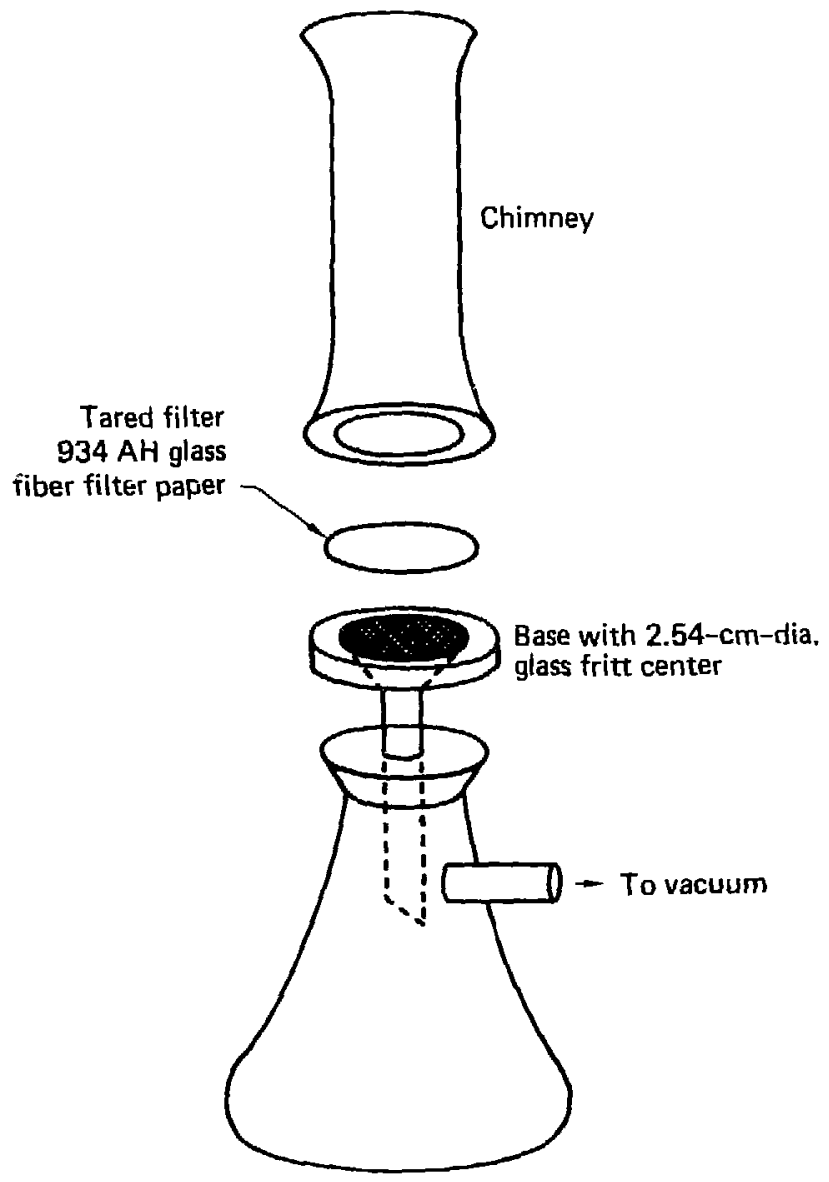

Figure 5 . The cesium filtering apparatus. 


\section{f. Uranium Purification 16}

1) Dissolve the sample in $1-2 \mathrm{ml}$ conc $\mathrm{HNO}_{3}$ with heat, and transfer with water to a $50-\mathrm{ml}$ glass centrifuge tube.

2) Aad $1 \mathrm{ml}(5 \mathrm{mg} / \mathrm{ml})$ lanthanum carrier to all uranium samples except those generated by the soil chemistry.

3) Make the sample basic with conc $\mathrm{NH}_{4} \mathrm{OH}$. Uranium is coprecipitated with the $\mathrm{La}(\mathrm{OH})_{3}$. Soil samples contain enough iron to coprecipitate uranium with $\mathrm{Fe}(\mathrm{OH})_{3}$ in those samples.

4) Heat the sample in an $80^{\circ} \mathrm{C}$ water bath for at least five minutes.

5) Centrifuge and discard the supernate.

6) Dissolve the $\mathrm{La}(\mathrm{OH})_{3}$ (or $\mathrm{Fe}(\mathrm{OH})_{3}$ ) with a minimum of $1 \mathrm{M} \mathrm{H}_{2} \mathrm{SO}_{4}$.

7) Dilute the solution to $5 \mathrm{ml}$ with $\mathrm{H}_{2} \mathrm{O}$.

8) Repeat steps 3 through 6 twice.

9) After discolving the $\mathrm{La}(\mathrm{OH})_{3}$ and diluting to $5 \mathrm{ml}$ a third time, dilute to $15 \mathrm{ml}$ with $0.06 \mathrm{M} \mathrm{H}_{2} \mathrm{SO}_{4}$.

10) Load a small ion exchange column to a height of $1 \mathrm{~cm}$ with preconditioned $A G 1-X 8,100-200$ mesh uranium anion exchange resin (sulfate form).

11) Condition the resin with $10 \mathrm{ml}$ of $0.06 \mathrm{M} \mathrm{H}_{2} \mathrm{SO}_{4}$.

12) Load the sample into the column.

13) When the liquid has run through the column, rinse the column reservoir four times with $5 \mathrm{ml}$ of $0.06 \mathrm{M} \mathrm{H}_{2} \mathrm{SO}_{4}$, allowing the reservoir to drain completely between rinses.

14) Wash the resin with $5 \mathrm{ml}$ of conic $\mathrm{HCl}$.

15) Wash the resin with $5 \mathrm{ml}$ of $6 \underline{\mathrm{M} \mathrm{HCl}}$.

16) Elute the uranium into $12-\mathrm{ml}$ conical centrifuge tubes with $10 \mathrm{ml}$ of $0.1 \mathrm{M} \mathrm{HCl}$.

17) Determine uranium in the ion exchange eluate by mass spectrometry. 
B. Tritium Analysis

A flow chart illustrating sample processing procedures used in tritiun analysis is shown in Fig. 7 on page 28 .

1. Air Moisture

a. Reservoir Preparation

1) Bake silica gel desiccant in a vacuum oven at $100^{\circ} \mathrm{C}$ for at least 24 hours, then cool and store in an argon atmosphere.

2) Fill a clean reservoir vessel (Fig. 6, below) with silica gel in an argon atmosphere.

3) Plug the neck with glass wool and seal the reservoir with a stopper.

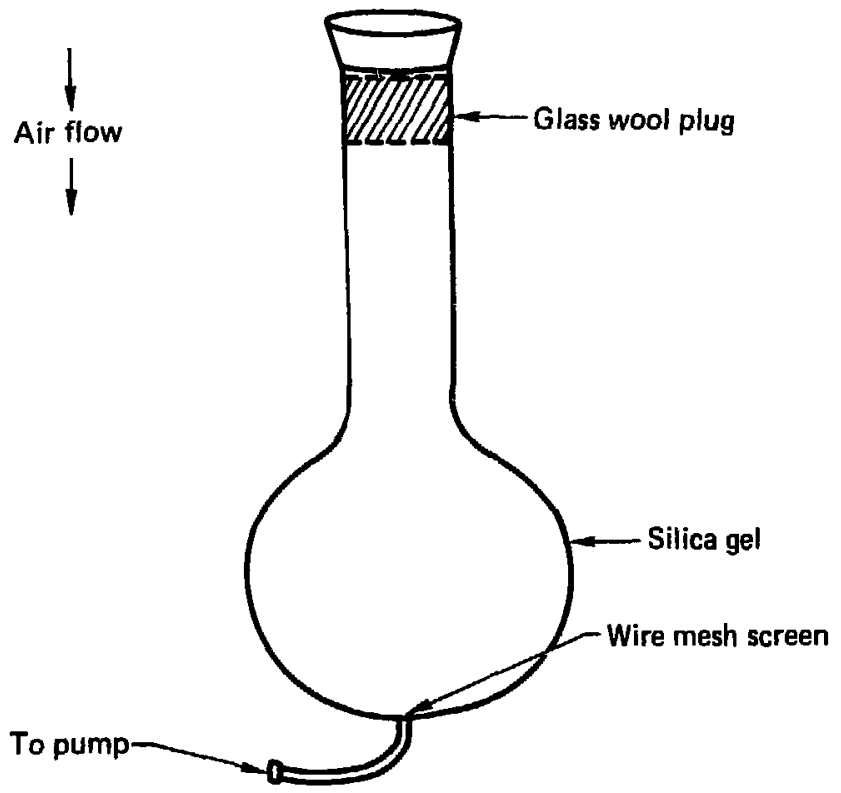

Figure 6. The silica gel reservoir. 


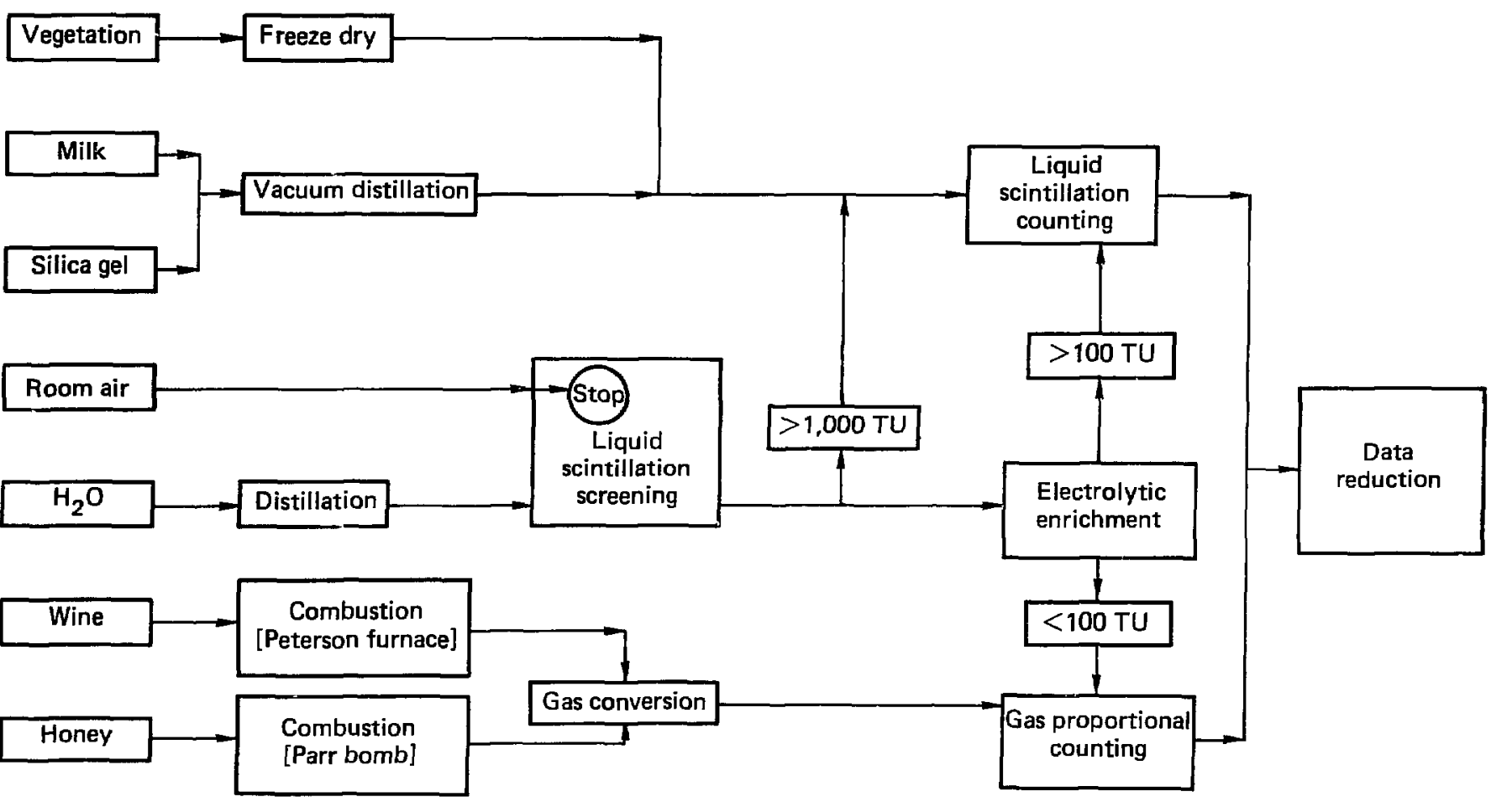

Figure 7. Low-level tritium determination. 
1) After the silica gel has been used to sample atmospheric moisture, determine the weight of the silica gel sample that is contained in the reservoir.

2) Mix the silica gel thoroughly to achieve homogeneity.

3) Remove an aliquot of about $300 \mathrm{cc}$ and determine its precise weight.

4) Transfer the silica gel aliquot to a freeze dry jar.

5) Construct the freeze dry assembly (Fig. 8, page 30):

Caution: Vacuum grease should not be used.

i) Attach the trap to the vacuum port with a triangle seal and three wing nuts.

ii) Plug the other trap opening with a rubber stopper.

iii) Evacuate the trap and check for leaks.

iv) Fill the trap's reservoir $1 / 3$ full with Dowanol (or equivalent).

v) Slowly add dry ice $\left(\mathrm{CO}_{2}\right)$ chunks to the reservoir until ful1.

vi) Insert a coarse $11.0-\mathrm{cm}$ filter paper and a 9-cm screen into the bottom of the adapter.

vii) Attach o-rings to the neck of the adapter and the jar. viii) Backfill the trap with argon and remove the stopper.

ix) Insert the adapter into the tra; while simultaneously opening the vacuum valve. Vacuum will hold the apparatus together.

6) Pump the assembly down, valve off, and check for leaks.

7) After the pressure has stabilized, envelop the jar in a heating mantle set for $100-110^{\circ} \mathrm{C}$. 


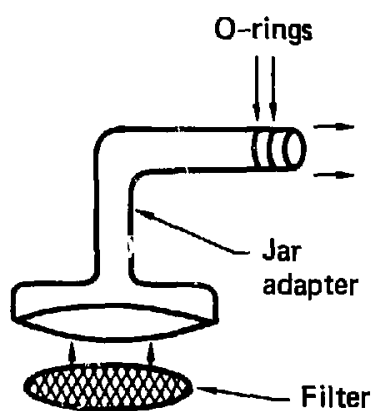

Filter paper and wire screen
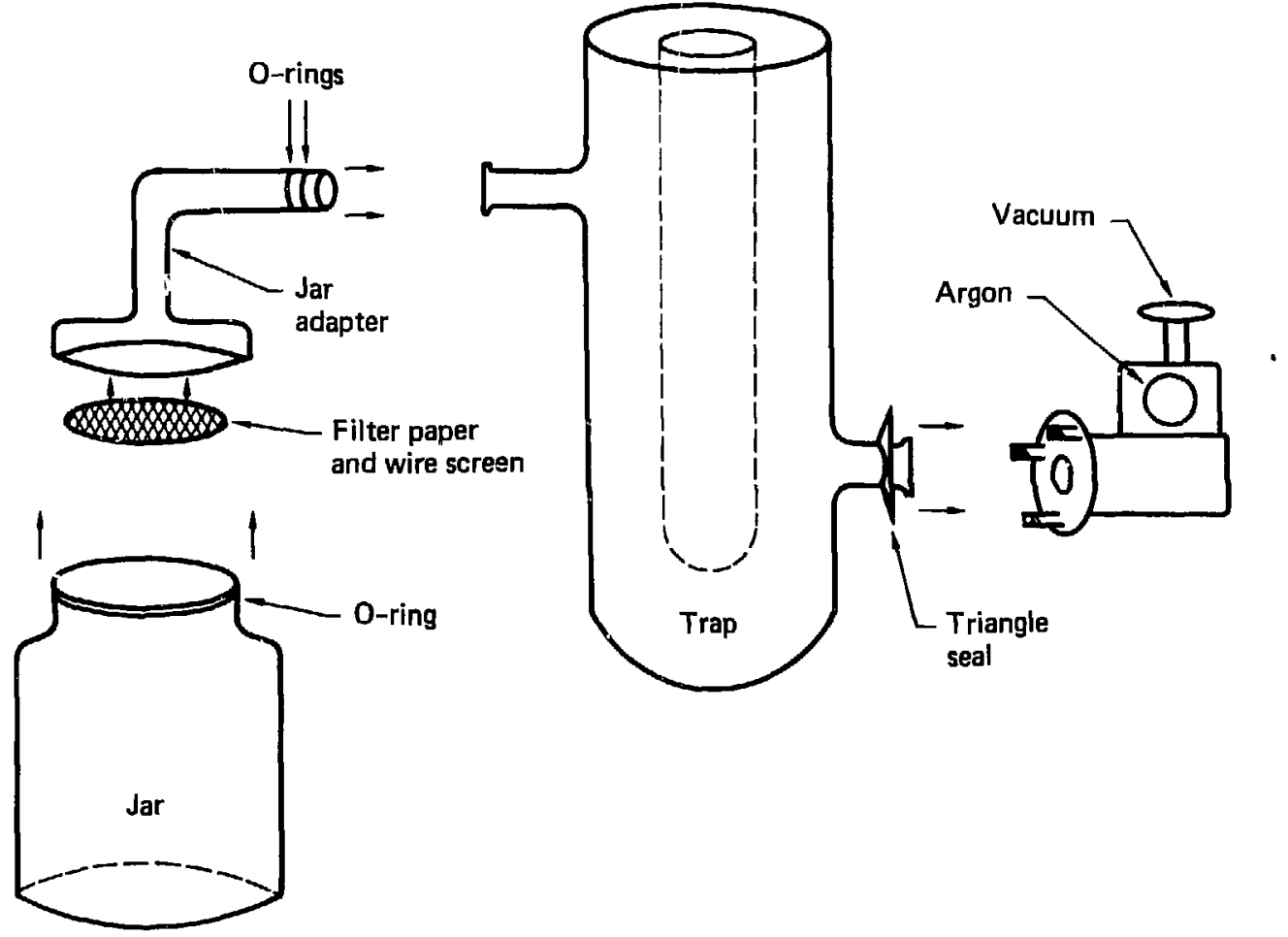

Figure 8. The freeze dry assembly. 
8) Continue to heat the sample for at least 24 hours. Add dry ice as needed and evacuate the freeze dry assemblies individually every two hours until the pressure stabilizes.

9) Backfill the trap with argon, remove the jar, and seal the opened trap end with a paraffin sheet.

10) Allow the silica gel to cool in a closed, tared container, and then reweigh to determine the amount of water lost.

11) Remove the Dowanol from the trap and collect the melt water from the ice that has formed on the cold finger.

12) Determine the tritium concentration in this water by liquid scintillation spectrometry.

\section{Water Samples}

The tritium concentration in water is normally too low to permit a direct liquid scintillation or proportional beta analysis. These samples must instead undergo a preliminary distillation to remove any physical or chemical contaminants followed by an electrolytic enrichment to increase the amount of tritium relative to the sample volume.

a. Distillation

1) Pour approximately $500 \mathrm{ml}$ of the water sample into a doublenecked, round-bottom boiling flask.

2) Through a distillation top, attach an argon line to the side neck of the flask and assemble the distillation apparatus (Fig. 9, page 32).

3) Attach the drain and water line to the condenser and check for leaks.

4) Turn on the heating mantle (set for $100-110^{\circ} \mathrm{C}$ ) and distill the entire sample. 
5) Transfer the distilled sample from the receiving flask to a clean bottle and seal.

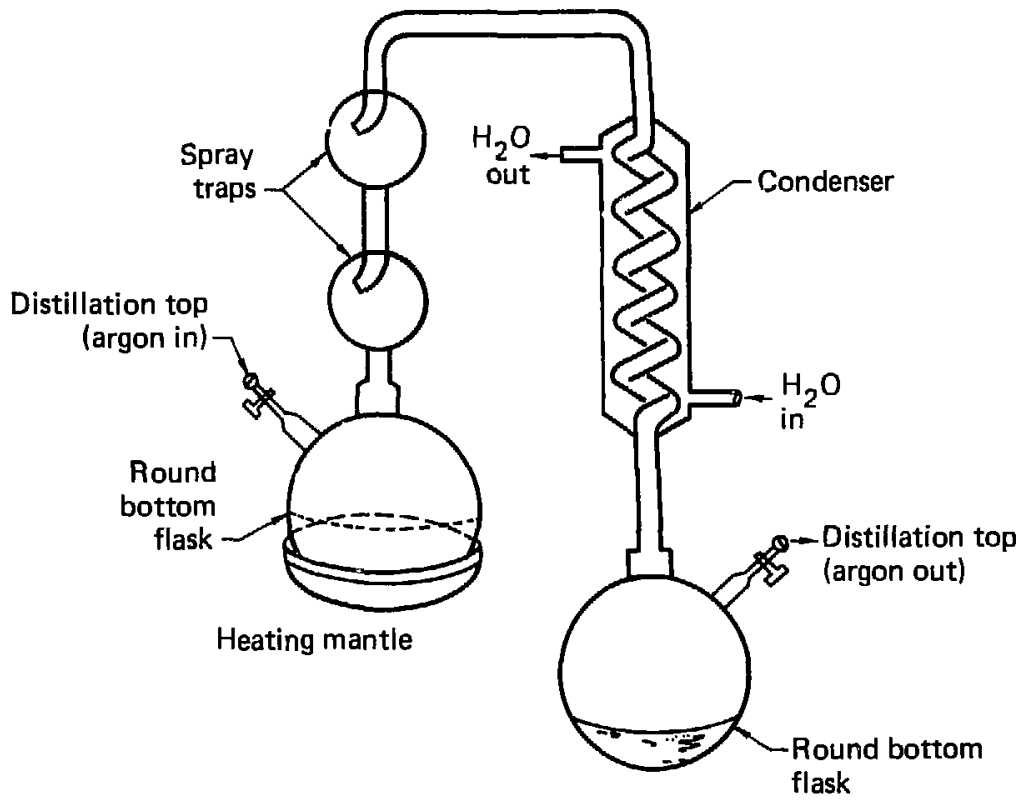

Figure 9. The water distillation apparatus.

b. Enrichnieit 6

The enrichment assembly (Fig. 10, page 33) consists of an iron cathode-nickel anode unit supported in a Pyrex cell by teflon baffle plates. Additional sample is entered into the cell from a top-mounted 250-ml delivery flask reservoir. During enrichment, the sample reservoir is under an argon atmosphere while the en.richment cell is maintained at a constant $6.0^{\circ} \mathrm{C}$ in a water bath. An enrichment set consists of two standards, a blank, and seven to nine samples. 


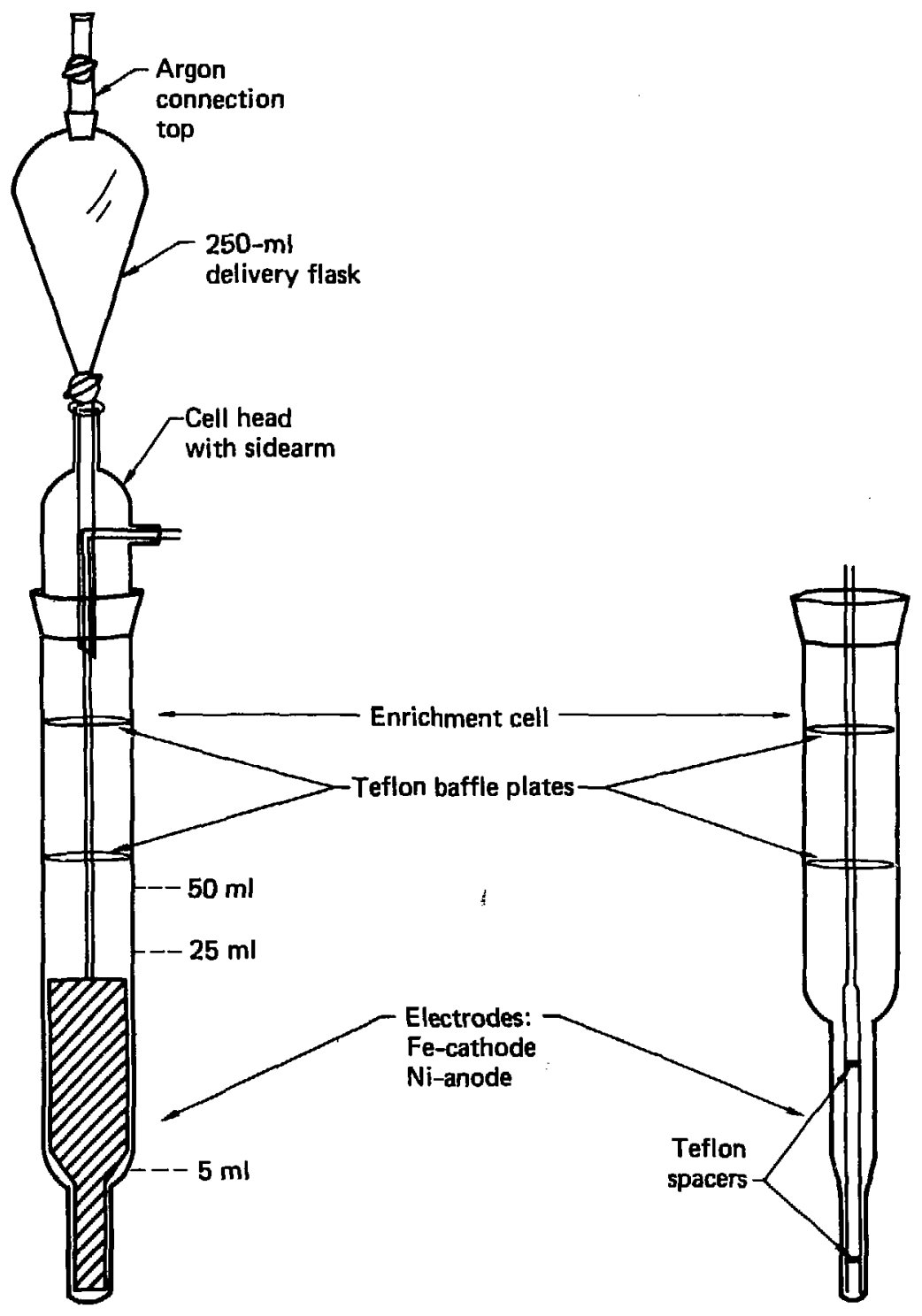

Figure 10. The enrichment cell assembly. 
1) Insert the electrode unit into the enrichment cell.

2) Add $1.5 \times 10^{12}$ moles of sodium hydroxide (as $1.6 \mathrm{ml}$ of $9.4 \underline{\mathrm{M}}$ $\mathrm{NaOH}$ ) to the cell.

NOTE: To avoid the tritium contamination of commercial reagent-grade sodium hydroxide, tritium free $\mathrm{NaOH}$ is synthesized by dissolving $1 / 4$ ib of sodium peroxide $\left(\mathrm{Na}_{2} \mathrm{O}_{2}\right)$ in $313 \mathrm{ml}$ of tritium-free water. The $\mathrm{NaOh}$ is stored in an argon atmosphere and periodically checked via gas conversion/gas proportional counting for its tritium level.

3) Measure a $250-\mathrm{ml}$ aliquot of the water sample.

4) Fill the enrichment cell to the 50-ml line and pour the remaining $200 \mathrm{ml}$ of sample into the delivery flask.

5) Complete the enrichment cell assembly pulling the electrode wires through the cell head sidearm. Do not use grease around any ground glass joints.

6) Immerse the enrichment cell in the $6.0^{\circ} \mathrm{C}$ water bath.

7) Connect the electrodes to a 3-amp constant current power supply.

NOTE: The electrolys is process at 3 amps evaporates about $1 \mathrm{ml}$ of solution every hour. It is therefore necessary to refill the cell to $50 \mathrm{ml}$ daily. When filling the cell, connect the argon supply to the argon connection top to exclude contaminated air from the assembly. If the cell cannot be filled every day (over the weekend for example), turn the power supply down to 1 amp.

8) When the sample reservoir is empty (8-12 days) and the cell volume is $25 \mathrm{ml}$, electrolyze at 0.3 amp to a volume of $5-7 \mathrm{ml}$ (about 10 days). 
9) When the 5-7-ml volume is attained, disconnect the power supply, remove the enrichment assembly from the water bath, and remove the delivery flask and cell head from the enrichment cell.

10) Neutralize the sample by bubbling carbon dioxide through the sample. This is done to release the hydrogen that is bound in the sodium hydroxide.

11) Attach the enrichment cell to a vacuum through a distil? ation head and a preweighed receiving bulb (Fig. 11, below).

12) Cool the bulb with dry ice methanol and evacuate the system.

13) Using a heating mantle, distill the sample in the enrichment cell, periodically relieving the pressure buildup in the system by opening the vacuum valve.

14) At completion, allow the receiving bulb to warm to room temperature. Reweigh to determine the final volume of water.

15) Enriched samples are analyzed for tritium by liquid scintillation spectrometry. A computer program, ENLIB4, is used to compute the enrichment factors which are entered into the final calculations.

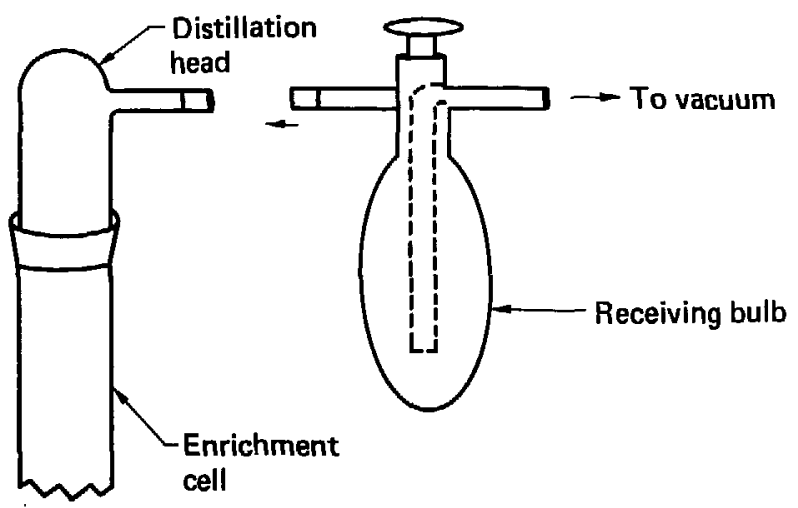

Figure 11. Enrichment cell head and receiving bulb for vacuum distillation. 


\section{Vegetation}

Vegetation samples are collected and analyzed quarterly. They are stored in plastic bags below $0^{\circ} \mathrm{C}$ to prevent loss or contamination of their water content.

1) Construct the freeze dry assembly (Figure 7, page 28) according to instructions in Section IIB -b (page 29).

2) As quickly as possible:

i. Remove sample from the freezer, cut top of the bag open, and weigh.

ii. Pack the bagged sample into the freeze dry jar.

iii. Attach the adapter to the jar.

iv. Backfill the trap with argon and remove the stopper.

v. Attach the jar and adapter to the trap, evacuate and valve off from the vacuum pump.

vi. Repeat for each sample.

The vegetation freeze drying process takes about four days to complete. During this time dry ice must be added to the coid fingers when needed and each sample assembly must be evacuated periodically and checked for leaks.

4) Upon completion, remove the jar by backfilling the trap with argon and seal the trap opening with a paraffin sheet.

5) Remove the Dowanol and dry ice from the trap. Allow the ice sample to melt.

6) Reweigh the bagged vegetation sample to determine the volume of water removed.

7) Transfer the ter sample from the trap to an appropriate sample container. 
8) If the volume of water in the sample exceeds $10 \mathrm{ml}$, tritium can be detennined by liquid scintillation spectrometry. Otherwise, it must be determined by gas proportional counting.

4. Goat Milk

1) Place $25 \mathrm{ml}$ of goat milk into a $50-\mathrm{ml}$ round bottom boiling flask.

2) Connect the boiling flask to an adapter and a receiving bulb (Fig. 12, below).

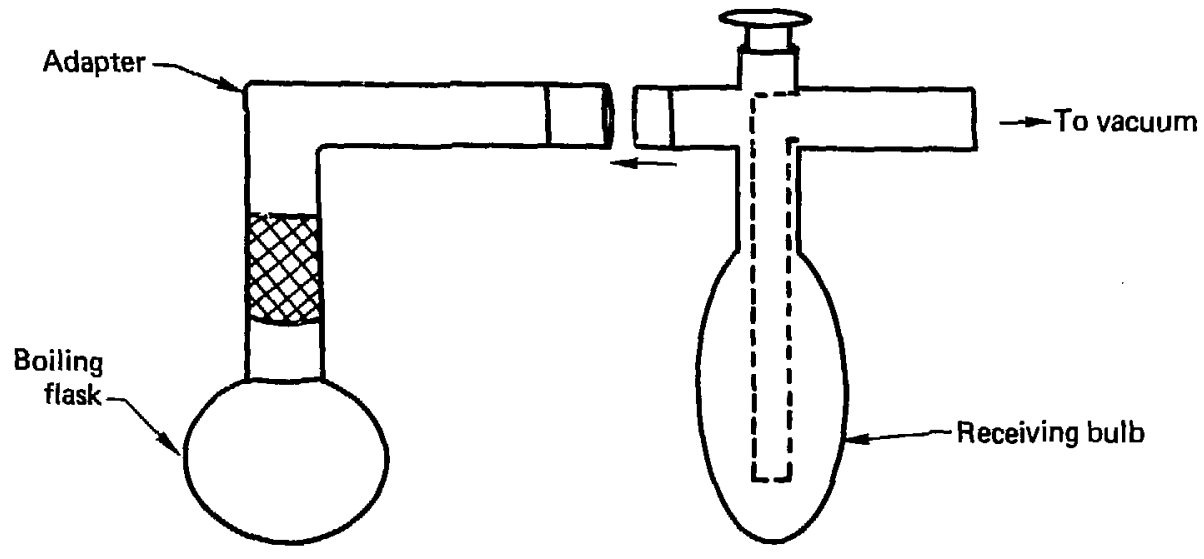

Figure 12. The milk distillation apparatus. 
3) Cool the bulb to $-78^{\circ} \mathrm{C}$ with dry ice/methanol and slowly evacuate the system, taking care not to splatter milk into the receiving bulb.

4) When the system is comp? ately evacuated, heat the boiling flask in a heating mantle.

5) When the milk is nearly dry, remove the heating mantle and isolate the receiving bulb.

6) Allow the bulb to warm to room temperature and transfer the water sample to a container.

7) Analyze the water for tritium by liquid scintillation spectrometry. 
III. QUALITY ASSURANCE PROCEDURES

A. Purpose

The quality assurance procedures of the Site Environmental Monitoring Group are designed to ensure that results of sample analyses are both accurate and reproducible. An additional objective of SEMP's quality assurance program is to be able to trace results to either stored samples or stored records. Included in "QA procedures," then, are storage of samples and sample data, processing of duplicate and standard samples, and verification of results.

B. Storage of Sample Data and Results

1. Heavy Elements Analyses

a. Sample Sheets

Laboratory worksheets are maintained for all samples. When a sample is received, initial information about the sample is recorded on the sheet. Sample number, date, type, and volume or weight, as well as the date received and the date "logged" onto the SEMP group's Framis data base ENV, are recorded. Any additional relevant information about the sample is also recorded. As the sample is processed, the chemical and counting procedures used are documented on the sample sheet, as are the chemist's initials and the date of the processing. Finally, any special infomation or unusual circumstances concerning the sample are recorded. Al1 sample sheets for the current year are stored in the laboratory, while those from previous years are archived.

b. Gamma Data

Results of gamma analysis are stored on the data base ENV and also on magnetic tapes. Computer printouts of gamma results generated by the gamma analysis program GAMANAL are also stored. 
c. Alpha Data

Results from plutonium alpha analys is are stored on the Framis data base ENV. Paper tape output containing alpha data and computer printouts containing both counting results and actual alpha spectra, all of which are generated by the alpha analysis program ALPHAQ, are stored permanently.

d. Beta Data

Cesium results determined by beta analysis are stored on the data base ENV. Computer printouts containing beta results of cesium analysis are stored permanently.

\section{e. Mass Spectrometry Data}

Uranium results determined by mass spectrometry are stored on the Framis data base ENV. Computer printouts containing these results are stored indefinitely.

Note that multiple methods of storage are used for all sample data. In addition, all samples with the exception of those analyzed for uranium are stored pemanently and maj be re-counted (see Section C, page 4l, for information on sample storage).

\section{Tritium Analysis}

a. Sample Sheets

As with heavy elements analysis, laboratory worksheets are maintained for all samples analyzed for tritium. Upon receipt of a sample, sequentially numbered worksheets are filled out with preliminary sample information. Type of sample, date of receipt, originator's identification number, and any additional relevant information concerning the sample are recorded on the sample 
sheet. All sample sheets are filled out in duplicate; one copy remains with the sample while it is being processed, while a carbor copy is kept separately in a worksheet notebook. As the sample is processed, procedural steps are noted on the worksheets. After processing, the original copy of the sample sheet replaces the carbon copy in the worksheet notebook. Original sample sheets are stored permanently.

b. Storage of Results

Results of all samples analyzed, either by gas proportional counting or liquid scintillation counting, are stored in a computer file called LOG. Scintillation counting results are also stored in a separate SEMP file called SCNSTR. Computer printouts of results determined by gas counting are stored with the actual sample sheets, while two different types of printouts containing liquid scintillation results are stored separately.

\section{Sample Storage}

1. Heavy Elements

Whenever possible, samples that have been processed and counted are stored in their final form. This ensures that samples may be re-counted and results re-calculated if there is any doubt as to the validity of original results.

a. Gamma samples, including milk solids, soil, and special samples, are stored indefinitely in their gamma cans.

b. Plated plutonium samples that have been alpha-counted are stored indefinitely.

c. Cesium samples are stored on beta-counted planchets. 
d. Samples analyzed for uranium cannot be stored since the samples are evaporated completely in mass spectrometry analysis.

\section{Tritium}

Samples analyzed for tritium are stored at least until analytical results have been obtained and verified, and for as long as is practical thereafter. Some samples may be stored in their original forms (e.g., wine, honey), while others cannot be and must be stored in some processed form (e.g., vegetation). When possible, a duplicate sample is taken initially to ensure that at a later point, a "duplicate" result may be obtained if necessary (see Section D3, page 45, for more information on duplication of samples).

a. Vegetation and Silica Gel Samples

Water collected froin these samples is stored five to six months for routine samples and one year for special samples.

b. Milk Samples

Water collected from the distillation of routine milk samples is also stored for five to six months.

\section{c. Water Samples}

Low-tritium water $(<1000 \mathrm{TU})$ is enriched prior to iiquid scintillation counting, and may therefore be stored in either the unenriched and enriched forms. Unenriched water samples are stored up to six months (the limitations here are space and equipment), while enriched samples are stored up to one year. Water that is higher in tritium content (> 1000 TU), which is not enriched, is stored up to six months after analysis is completed. 
d. Wine and Honey Samples

Wine and honey samples are stored in their original form for at least six months.

\section{D. "QA" Samples}

1. EML Samples

Samples are received from the EML (U.S. Department of Energy Environmental Measurements Laboratory, New York, NY) approximately every six months and are analyzed for a variety of nuclides. Types of samples analyzed include soil, water, air filters, and vegetation. The procedures used for analyzing the soil samples are identical to those described in Section II, Methods of Sample Preparation and Analysis, for routine soil samples. Procedures used in the analysis of the EML air filters, vegetation, and water samples do not, however, exactly follow the routine procedures given in Section II, but require special processing as follows.

\section{a. EML Air Filters}

Air filters are first gamma counted in a plastic petri dish. One half of the dish is nested inside the other half, enclosing the air filter. Additional glass fiber filters are enclosed on the non-counter side to ensure that the filter to be counted is held in tightly. The entire apparatus is then taped and gamma counted. After counting, the chemical procedures used follow those used for routine samples (see section IIA-2, page 8). 
b. EML Vegetation Samples

The procedures used in processing vegetation are basically the same as those used in processing routine LLNL perimeter air filters. First, a pre-determined amount of sample is weighed out; it should contain approximately the same amount of plutonium as the tracer to be added. The approximate amount of plutonium in the sample is indicated on the information sheet accompanying the EML samples. Tracer is auded, and the sample is dried at $105^{\circ} \mathrm{C}$ for at least four hours and then ashed at $475^{\circ} \mathrm{C}$ for at least two days. The "wet ash" procedure using $\mathrm{HNO}_{3} / \mathrm{HF}$ followed by $\mathrm{HNO}_{3} /$ $\mathrm{HClO}_{4}$ (see Section IIA-2, page 8) is then performed twice in order to fully digest the sample.

c. EML Water Samples

Three 7-ml aliquots are removed from the 1-1 water sample and analyzed for tritium by liquid scintillation counting. The remaining water is analyzed for heavy elements, ordinarily only plutonium and cesium. The sample is diluted to four gallons total with distilled water and then analyzed according to routine procedure (see Section IIA-3, page 11). A blank, containing four gallons of distilled water, is analyzed concurrently.

\section{d. Storage of EML Results}

Computer printouts of all EML results are stored indefinitely. EML results are also entered into the data base ENV, in a table designated specifically for EML data. 
2. EPA Samples

EPA water samples (U.S. Environmental Protection Agency Environmental Monitoring Systems Laboratory, Las Vegas, NV) are received every two months. They are analyzed only for tritium, by a direct liquid scintillation count; no distilling or enriching is involved. Results are stored in the SEMP computer files LOG (containing all tritium analysis results) and SCNSTR (containing all scintillation count results). Computer printouts containing all scintillation count results include EPA results.

3. Duplication of Samples

All types of samples are duplicated periodically to ensure reproducibility of results. Some samples (ine., vegetation and other samples analyzed by freeze-drying) are always duplicated so that an alternate sample may be run in case of a processing error.

\section{a. Heavy Elements}

1) Air Filters

A duplicate sample is taken at one air filtering station every other month. Since there are six perimeter air filtering stations, and the extra sampler is moved to a different site bimonthly, all sampling stations are checked once a year.

2) Water Samples

Daily water samples are duplicated once weekly. These duplicate samples are analyzed separately.

\section{3) Soil Samples}

At least ten percent of the annual soil samples received are duplicate samples. These duplicates are analyzed with the original soil samples. 
b. Tritium

1) Vegetation

Vegetation is sampled quarterly, and two vegetation samples are taken at each sampling location. Duplicate samples are not routinely analyzed, although one such sample is analyzed quarterly as a $Q A$ "check".

2) Silica Gel Samples

A duplicate sample is collected and analyzed from a different station each month.

3) Water Samples

At least $10 \%$ of all water samples submitted for SEMP analysis are duplicate samples.

4. Internal Standards and Blanks

a. Heavy Elements

1) Air filter standards prepared with stock solutions of plutonium and uranium are analyzed with routine air filters at least every six months. Air filter blanks are included with every month's air filter samples, and are analyzed monthly with the routine samples.

2) Water blanks are normally anaiyzed every other month; water standards are analyzed biannually. EML water samples, which are analyzed along with a "blank" (see Section IIID-1, page 43), suffice as biannual standards.

3) Soil samples, analyzed annually are first gamma counted, then processed chemically. An NBS soil sample is run as a gamma 
standard, but is not analyzed chemically. A blank comprising "washed and ignited" sand (Mallinckrodt) is run with the routine soil samples being processed.

b) Tritium

Tritium standard solutions of approximately $5.5 \times 10^{-3} \mathrm{Ci} / \mathrm{ml}$ are made at various water dilutions and serve as standards for liquid scintillation counting. Another standard solution of approximately $8.3 \times 10^{-6} \mathrm{Ci} / \mathrm{ml}$ is made up from a stock solution, and is run with each enrichment set. This standard is also used as a gas proportional counter/liquid scintillation counter crosscheck.

Dead water ( $<3$ TU) is used as a blank for enrichment sets. Hydrogen gas is used to determine background in the gas counter.

\section{E. Verification of Results}

Once processing of a sample is completed, the result is checked against past results for the same type and location of sample. Any significant discrepancy here may necessitate recounting or possibly even reprocessing of the sample. The flow of sample data is also checked to ensure that no mistakes were made in transferring information, i.e., that information on the sample sheet was transferred correctly to computer input, and that al1 information has been "logged" onto the data base correctly. 


\section{REFERENCES}

1. W. J. Silver, C. L. Lindeken, K. M. Wong, A. Conover, J. H. White, Environmental Monitoring at the Lawrence Livermore Laboratory 1978 Annua) Report, Lawrence Livermore National Laboratory, Report UCRL-50027-78 (1979).

2. D. C. Camp, C. Gatrousis, L. A. Maynard, "Low Background Ge(Li) Detector Systems for Radioenvironmental Studies," Nucl. Instrum. Meth. 117, 189 (1974).

3. R. Gunnink, J. B. Niday, Computerized Quantitative Analys is by GammaRay Spectrometry, Lawrence Livermore National Laboratory, Report UCRL5106) (1974).

4. J. H. Dellis, A Low Background Beta Counter for Use in Ärit.i-coincidence with the Amperex Cosmic Ray Shield, Lawrence Liverinore National Laboratory, Report UCRL-6429-T (1961).

5. W. E. Nervik, Brunhilde - A Code for Analyzing Multicomponent Radioactive Decay Curves, Lawrence Livermore National Laboratory, Report UCRL6937-T (1962).

6. H. G. Ostlund, E. Werner, "The Electrolytic Enrichment of Tritium and Deuterium for Natural Tritium Measurements," Tritium in the Physical and Biological Sciences, Proceedings of symposium organized jointly by the IAEA and the Joint Commission on Applied Radioactivity (Series ST 1/Pub/39), Vienna, May 1961 .

7. E. H. Willes, Lawrence Livermore National Laboratory. To be published.

8. A. E. Bradley, E. H. Willes, "A Risetime Discriminator for Low Level Tritium Counting, IEEE Transaction on Nuclear Science, February 1973.

9. W. H. Hutchin, A. E. Bradley, E. M. Morimoto, W. Wadleigh, E. H. Willes, A Facility for the Determination of Low Level Tritium in Biological and Environmental Samples, Lawrence Livermore National Laboratory, Report UCRL-73128 (1971).

10. I. A. Dupzyk, R. J. Dupzyk, Separation of Uranium from Urine for Measurements by Fluorimetry or Isotope-Dilution Mass Spectrometry, Lawrence Livermore NationaT Laboratory, Report UCRL-75768 (1978).

11. K. M. Wong, G. S. Brown, V. E. Noshkin, "A Rapid Procedure for Plutonium Separation in Large Volumes of Fresh and Sal ine Water by Manganese Dioxide Coprecipitation," J. Radioanal. Chem. 42, 7 (1978).

12. J. Van R. Smit, W. Robb, J. J. Jacobs, "Cation Exchange on Ammonium Molybdophosphate-I," J. Inorg. Nucl. Chem., 12, 104 (1959).

13. "Radiochemical Determination of Plutonium in Soil by Leaching," USAEC Health and Safety Laboratory, Manual of Standard Procedures, 3rd ed., NYO-4700 (1970). 
14. N. A. Talvities, "Electrodeposition of Actinides for Alpha Spectrometric Determination," Allal. Chem. 44280 (1972).

15. R. F. Mitchell, "Electrodepositon of Actinide Elements at Tracer Concentrations," Anal. Chem. 32326 (1960).

16. R. D. Carver, R. S. Newbury, N. A. Jones, "Determination of Smali Amounts of Uranium Isotopes in Geologic Samples Which Have Been Perturbed by Nuclear Explosions," (U) Lawrence Livermore Laboratory Report UCRL-50607 (1969). 